
Q
354
A65
1915
MOLL 


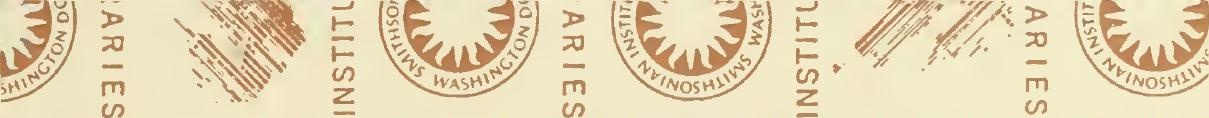

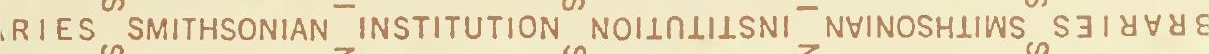

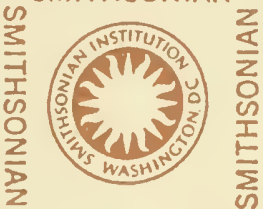

(n)
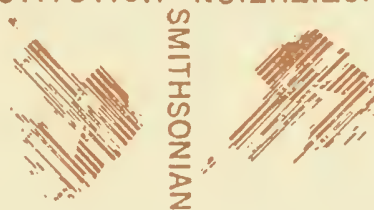

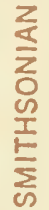

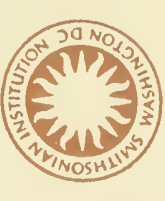

$\frac{1}{3}$
$\frac{1}{1}$
$\frac{1}{5}$
0
$\frac{2}{2}$
2

INSTITUTI

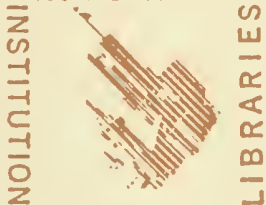

SMITHSONIAN
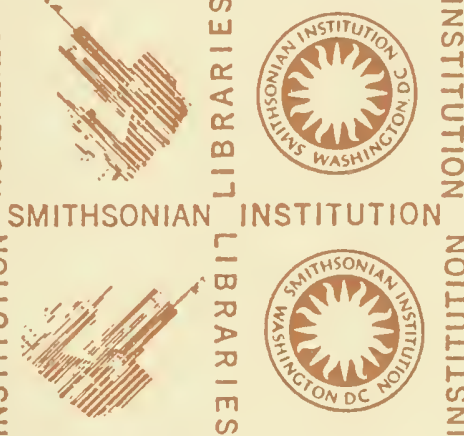

$\sum_{0}$
$=1$
$\bar{c}$
$\overline{0}$

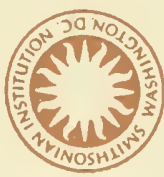

$\frac{1}{\infty}$

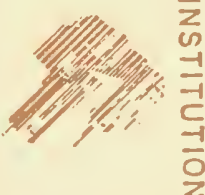

$z$
c
$\overline{-1}$
$\bar{c}$
$\frac{-1}{0}$
$\frac{2}{z}$

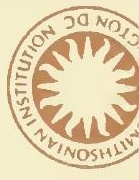

$S \exists 18 \forall y$
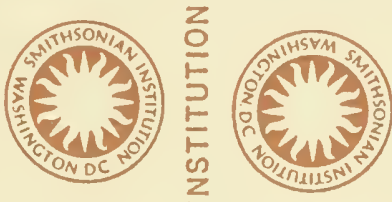

5
0
0
2
$\frac{D}{m}$
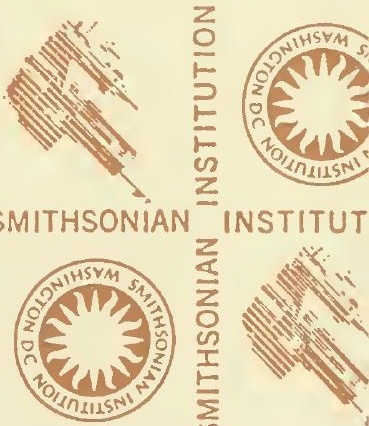

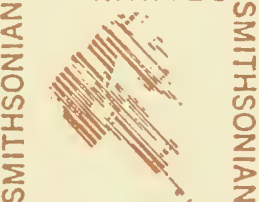

NOILIIILSN

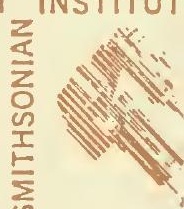

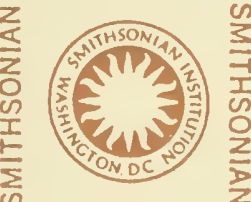

SMITHSONIAN

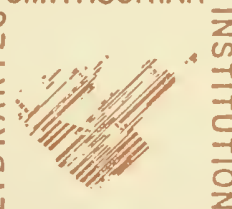

NHINOSHLIWS

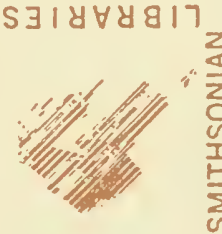

INSTITUTION
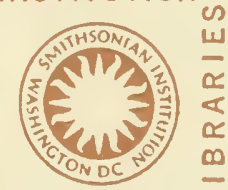

S $318 \forall 8817$
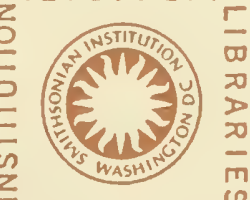

TITUTION

NHINOSHIIWS
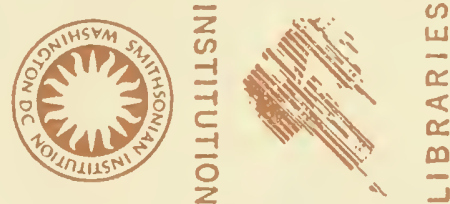

SMITHSONIAN

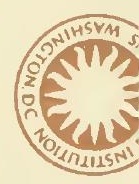

LIBRARIES
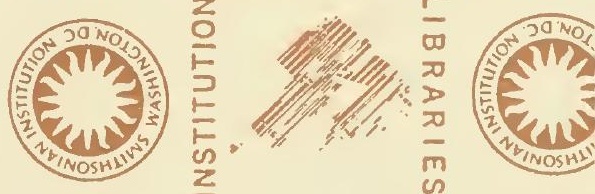

S $\exists \mid y \forall y$
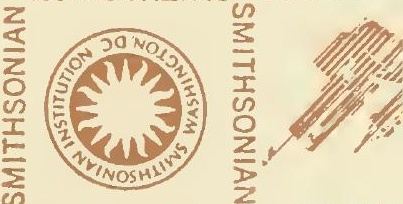

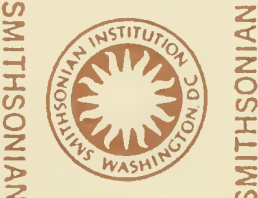

NYINOSHIINS 

Apstim, Pros. C.

$$
\begin{aligned}
& \text { Sitzungsberichte } \\
& \text { der } \\
& \text { Gesellschaft Division of moly } \\
& \text { Maturforschender Freunde } \\
& \text { zu Berlin. }
\end{aligned}
$$

Nr. 5.

\section{INHALT:}

Nomina conservanda./Unter Mitwirkung zahlreicher Spezialisten lerausgegeben von Prof. C. Arstein, Berlin . . . . . . . . . . . 119 Zweite wissenschaftliche Sitzung am 18. Mai 1915 . . . . . . . . . 202

$$
\text { Reed Dec, } 27 / 15
$$

\section{op. adopted in Gfficial List}

\section{is Div conwibutst}

\section{SMITHSONIAN}

FEB 291988

\section{BERLIN.}

In Kommission bei R. Friedländer \& Sohn,

NW Carlstrasse 11. 


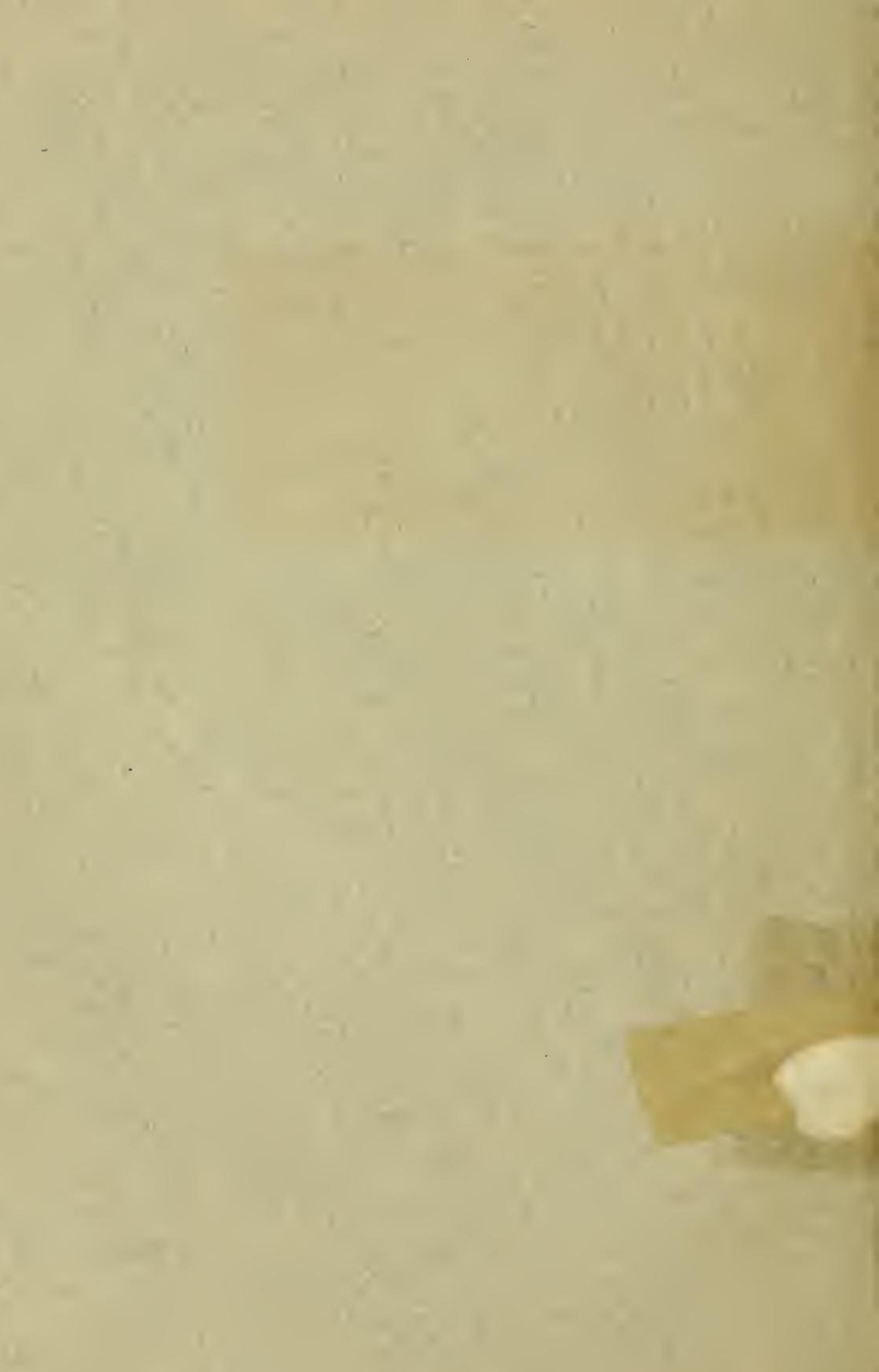




$$
\begin{aligned}
& \text { Ales: } \\
& \text { herlin NiS } \\
& \text { Femingyír 5? }
\end{aligned}
$$




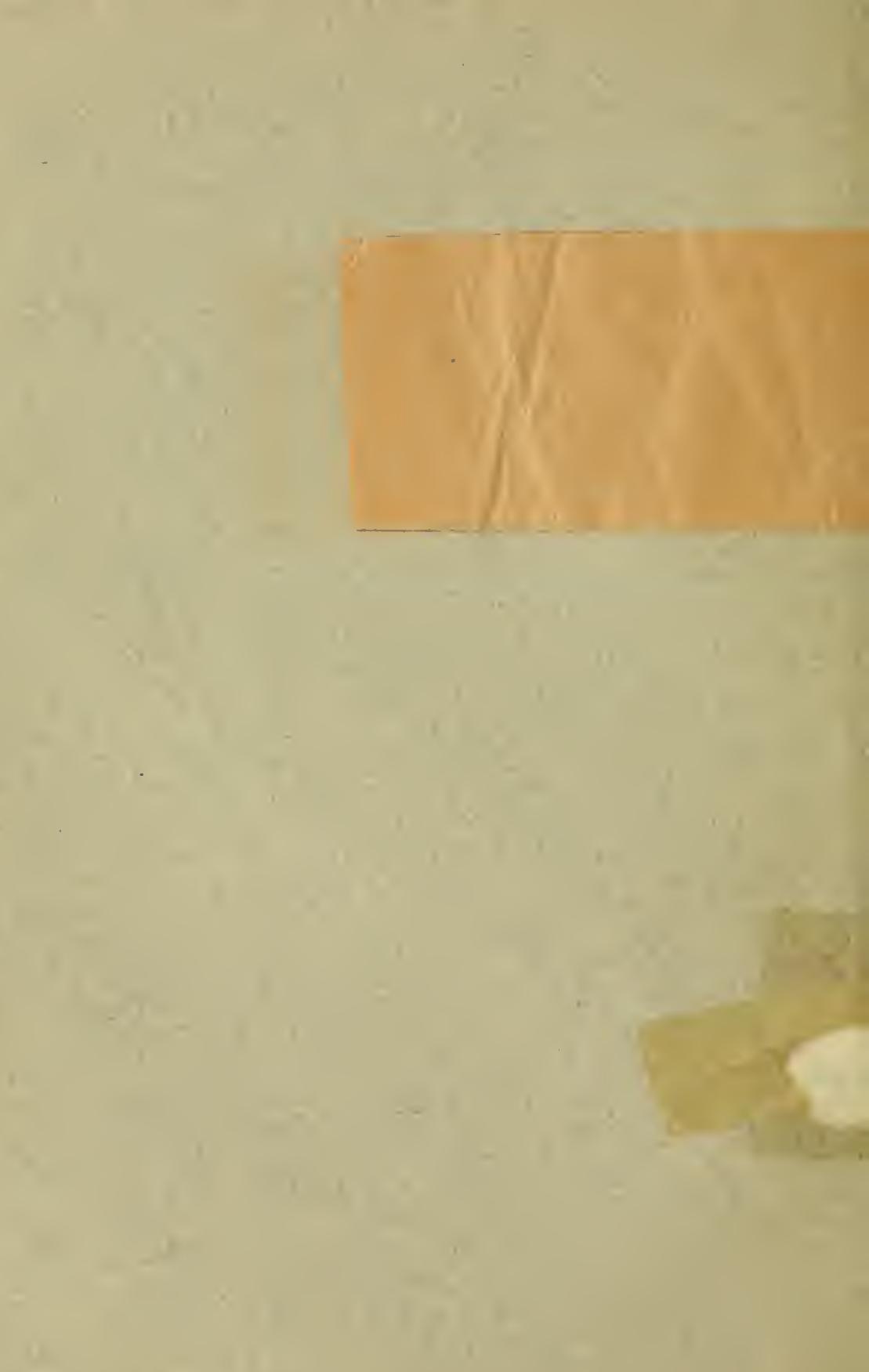




\author{
Sitzungsbericht \\ der
}

Gesellschatt naturforschender Freunde. zu Berlin

vom 11. Nai 1915.

Vorsitzender: Herr R. Heymons.

Herr 0. HeInRoth sprach über Entwicklungsreihen und Aufnahmen einheimischer Vögel.

\title{
Nomina/ conservanda.
}

\section{Unter Mitwirkung zahlreicher Spezialisten}

herausgegeben von Prof. C. A Apśters, Berlin.

Die strikte Anwendung des Prioritätsgesetzes hat zu so zahlreichen — bei Aufstellung des Gesetzes wohl nicht vorausgesehenen - Änderungen allbekannter Tiernamen gefülırt, daß sich eine große Zahl von Zoologen dagegen ausgesprochen hat (vgl. z. B.: Zool. Anz., v. 39, 1912, p. 56-62, 365-366; v. 40, 1912, p. 157-160, 207-208; Verh. D. zool. Ges., v. 21, 1912, p. 227). Auf dem 9. Intern. Zool. Kongr. in Monaco 1913 (Rennes 1914 p. 890) ist dank dem Vorgehen von A. Brader beschlossen worden, Ausnalimen (suspensions!) vom Prioritätsgesetz zuzulassen. Anträge zu solchen Ausnahmen sind mehrmals an die Intern. Nomenkl.-Komm. eingereicht worden (Zool. Anz., v. 44, 1914, p. 238-240, 630-632). Ferner werden "om Sekretär der Intern. Nomenkl.-Komm. Herru Prof. Stules sog. "Otficial lists of generic names", zusammengestellt von Spezialisten resp. Spezialkommissionen, herausgegeben (Zool. Anz., v. 39, 1912, p. 62, 557, 653 und Congr. int. Zool., v. 9, 1914, p. $858 \mathrm{ff}$.$) . Durch$ letztere Listen werden Namen - noch dazu in Übereinstimmung mit dem Prioritätsgesetz — festgelegt. Neue, erst im Manuskript herumgesandte Listen enthalten dazu größtenteils Namen, die nur für den Spezialisten Interesse haben, den übrigen Zoologen aber vollkommen unbekannt sind (z. B. Crustaceen, Crinoiden), während gerade die allgemein bekaunten Namen fehlen. Durch 
diese Listen ist eine Konstanz der Namen in weiteste Ferne gerückt und eine Erhaltung bekannter Namen nicht gewährleistet.

Ich entschloß mich daher, nach Rücksprache mit zahlreichen Kolleger, eine Liste der "Nomina conservanda“ unter Mitarbeit von Spezialisten herauszugeben. Durch diese Liste wird eine Basis für weitłre Verhandiungen gegeben, deren Resultate dem nächsten Intern. Zool.-Kongreß (i) vorgelegt werden können. Durch Annahme der Liste würde eine Konstanz der allbekannten Namen mit einem Schlage gegeben.

Die Liste ist am 13. VII. 1915 als Antrag an den Sekretär der Intern. Nomenkl.-Komm. Herm Prof. StiLEs gesandt worden. Nachträglich sind nur kleine Änderungen vorgenommen worden, nur die Liste der Araneida ist nel aufgestellt.

$\mathrm{Zu}$ den Listen möchte ich folgendes bemerken:

Außer dem Namen der zu schïtzenden Gattung ist eine Art genannt, für welche die Gattung erhalten bleiben soll. Natürlich sind die Listen der D. Zool. Ges. (Zool. Anz., v. 40, 1912, p. $155 \mathrm{ff}$.) und von HandLIRSCH (Handb. Ent., v. 3, 1913, p. 79-99) ansgiebig benutzt worden. In die Liste sind nicht nur die Namen aufgenommen worden, die in Gefahr sind geändert zu werden, sondern auch andere bekannte Genera, da für diese jederzeit auch die Gefahr der Änderung eintreten kann. Aber auch rein Linnésche Gattungen finden sich in der Liste, so daß letztere zugleich ein Verzeichnis derjenigen Namen darstellt, die allgemein bekannt sind. In einigen Fällen sind bei einer Gattung mehrere Arten genannt; die kursiv gedruckten bedenten, daß nur der Artname als solcher geschützt werden soll, ohne Rücksicht anf die Gattung. Die Literatur gibt meist leicht zugängliche Werke an, in denen sich die betreffenden Gattungen und Arten beschrieben finden oder in denen sich Literaturhinweise finden. Der Anordnung der Gruppen ist meist Clatus, Lehrb. Zool., ed. 2, 1883, zugrunde gelegt.

Schließlich spreche ich allen den Herrn Kollegen, die die Listen verfaßt haben oder mit ihnen einverstanden sind und deren Namen zum 'Teil am Schlusse der betreffenden Gruppe genannt sind, sowie denen, die mich so kräftig unterstiitzt haben, meinen besten Dank aus, ebenso der Gesellschaft naturforschender Freunde, daß sie die umfangreiche Liste in ihren Sitzungsberichten zum Abdruck gebracht hat. 


\section{Protozoa.}

Foraminifera.

Amoeba ...... Ehrbg. 1831 proteus...... P. Pall. 1766

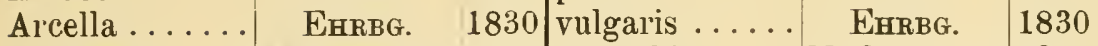
Cornuspira .... M. Schultze 1854 planorbis ..... M. Schultze 1854 Difflugia..... Lecherc 1815 globulosa ..... Globigerina ... ORB. 1826 bulloides...... Gromia...... DUJ. 1835 oviformis ..... Lagena ...... WALK. \& $\mathrm{J}_{\text {AC. }} 1784$ vnlgaris ...... WILLIAMSON 1858 Miliola ....... Lm. 1804 cyclostoma.... M. Schultze 1854 Nummulites ... Orbulina...... Polystomella. . Rotalia $\ldots . .$. Textularia ....

LM. 1801

OrB. $\quad 1839$ universa...... LM. $\quad 1822$ striato punctata LM. $\quad 1801$ beccarii ...... DEFR. 1828 agglntinans ...

Lit.: Bötrchl in: Brons's Kl. Ord., v. 1 1880-1889.
DuJ. $\quad 1837$

ORB. 1826

DUJ. 1835

\begin{tabular}{c|c} 
OrB. & 1839 \\
F. \& M. & 1866 \\
L. & 1758 \\
OrB. & 1839
\end{tabular}

\section{Heliozoa.}

\begin{tabular}{|c|c|c|c|c|c|}
\hline Acanthocystis & Cart. & 1863 & |spinifera...... & GrEEFF & 1869 \\
\hline Actinophrys ... & EhrbG. & 1830 & sol . . . . . . & EhrbG. & 1830 \\
\hline $\begin{array}{l}\text { Actino- } \\
\text { sphaerium ... }\end{array}$ & F. ST. & 1857 & eichhorni ..... & ЕнRвG. & \\
\hline Clathrulina.... & Crenk. & 1867 & |elegans....... & Cienk. & 1867 \\
\hline
\end{tabular}

Lit.: Bürschul in: Brons's Kl. Ord., v. 1 1880-1889.

Pénard, Helioz. eau douce, 1904.

\section{Radiolaria.}

Acanthometra . Aulacantha ... Anlosphaera... Challengeron . . Collosphaera . Collozoum .... Encyrtidium . Heliosphaera . . Physematium . . Sphaerozoum . . Thalassicolla . . Tuscarora ....
J. MüLL.

H.

$\mathrm{H}$.

H.

J. MÜLL.

$\mathrm{H}$.

EhrbGr.

H.

Meyen

Meyen

Huxl.

Murr. 1862
1847

1862

1834 at 1834 p

$18 \overline{1}$ nncleata ......

1885̄|tetraëdra .....
J. MÜLL.

H.

H.

MURr.

J. MÜLL.

J. MÜLL. H.

$\left.1855^{1}\right)$

1860 1860

1885

1855

1856

1860

1860

\begin{tabular}{c|c} 
H. & 1860 \\
MEYEN & 1834
\end{tabular}

J. MÜLL. 1858

Huxu.

MURR.
1851

1885

\section{Lit.: Harckrt in: Rep. Voy. Challenger, v. 18 pars 401887.}

1) HaEckel, Radiolarien, 1862. 
Sporozoa.

Adelea ....... Amme Scrn. 1875|0vata ........

Coccidium

Leuck. 1879 cuniculi

Amate Schn. 1875

Eimeria

AIMÉ Schn.

Cregarina

Dur.

187.

Rivolta

1878

Eimer 1870

Monocystis ....

F. ST.

BÜTSCH.

NäGELI

Nosema

1828 ovata

DuF.

1828

DUJ.

1835

Myxobulus ....

Plasmodium ... MARCH. \&

1882 min

BÜтsCH.

1882

Monz.

1887

\section{CELLI}

Porospora ....

Sarcocystis....

Ainé Schn. 1875

LANK.

1882 mischeriana ...

Lav.

1885 malariae ......

Lit.: LABBE in: Tierreich, v. 51899.

Flagellata.

\begin{tabular}{|c|c|c|c|c|c|}
\hline Anuphisolenia. & F. ST. & $\mid 1883$ & |globifera & F. St. & $\left.1883^{1}\right)$ \\
\hline Ceratium ... & SchraNk & 1793 & tripos .. & Nitzsch & $\left(817^{1}\right)$ \\
\hline eratocorys & F. ST. & 1883 & horrida... & F. ST. & $\left.1883^{1}\right)$ \\
\hline ercomollas & DuJ. & 1841 & intestinalis . & LAगIBL & \\
\hline ostia .... & LECLERQ & 1890 & necatr & Henneg. & 884 \\
\hline 10pnysis & EнRBG. & 1839 & acuta & EHRBG. & $839^{1}$ \\
\hline roll & ЕнввG. & & laria.. & EHRBG. & \\
\hline 11. & EHRBG. & 1830 & viridis. & SCHRANK & \\
\hline inium & F. ST. & 18 & fus & ЕнRвG. & $3^{1}$ \\
\hline $\mathrm{ca} \ldots$ & SURIR. & & mi & Surir. & \\
\hline ercus & F. ST. & & ficus . & F. St. & \\
\hline er & EHrbG. & & gens. . & ЕнRвG. & $\left.10^{1}\right)$ \\
\hline entrum & ЕнгвG. & & $\mathrm{mi}$ & ЕнRвG. & 33 \\
\hline & Murr. & & pseudonoctiluca & MURR. & 187 \\
\hline & J. ClankK & & & & \\
\hline richomonas . & DoNnÉ & & vaginalis & DONNÉ & \\
\hline & GrUBY & & is ... & GRUBY & \\
\hline rolyox. & L. & & globator .... & L. & 1758 \\
\hline
\end{tabular}

Lit.: BÜтschli in: Brons's Kl. Ord,, $v .1$ 1880-1889.

1) Schütt in: Engler \& Prante., Nat. PAlanzenfam., II 1896.

\section{Ciliata.}

Acineta

EHRBG.

|1833 |mystacina

Ehrbg.

1831

Amphileptus .. EHRBG.

Balantidium... CLa . \&

Bursaria LАCHM.

Carchesium ...

MÜLL.

1830 fascicola . . . . . .

MÜLL.

1773

Malmst. 1857

Chilodon..... EhrbG.

Ehrba.
Ehrba.

1773

1830 polypinum

1833 cucullus
MÜLL.

L.

MÜLL. 


\begin{tabular}{|c|c|c|c|c|c|}
\hline Colpoda & MÜLL. & 1773 & |cuculus . & MÜLL. & 1773 \\
\hline Cyttarocylis.. & FoL & 1881 & cassis ...... & H. & 1873 \\
\hline Dictyocysta ... & EHrBG. & 1854 & lelegans ...... & EHrbG. & 1854 \\
\hline Entonidium ... & F. $\mathrm{ST}_{\mathrm{T}}$ & 1859 & caudatum .... & F. ST. & 1859 \\
\hline Epistylis .... & Ehrba. & 1830 & plicatilis ..... & Енввя. & 1831 \\
\hline Euplotes..... & EHRBG. & 1831 & patella ....... & MÜLL. & 1773 \\
\hline Folliculina ... & Lм. & 1816 & ampulla .... & MÜLL. & 1786 \\
\hline Glancoma . . . . & Енввя. & 1830 & scintillans . . & Енгвя. & 1830 \\
\hline $\begin{array}{l}\text { Ichthyophthi- } \\
\text { rius } \ldots \ldots \ldots\end{array}$ & FoUQUET & 1876 & & FoUQUET & 376 \\
\hline Opalina.... & $\begin{array}{l}\text { PURK. \& } \\
\text { VALENT. }\end{array}$ & 1835 & ranarum & $\begin{array}{c}\text { PURK. \& } \\
\text { VALENT. }\end{array}$ & 183 \\
\hline Oxytricha ... & Bory & 1824 & gibba . . & MÜLL. & 1786 \\
\hline Paramaecium . . & $\mathrm{F} . \mathrm{S}_{\mathrm{T}}$ & 1860 & aurelia... & MÜLL. & 178 \\
\hline Podophrya ... & EHRBG. & 1833 & cyclopum & $\begin{array}{l}\text { ClaAP. \& } \\
\text { LACHM. }\end{array}$ & 18 \\
\hline Spirostomum & EHRBG. & 1835 & ambigulum & BORY & 182 \\
\hline Ste & OKEN & 1815 & polymorphus . & MÜLL. & 1773 \\
\hline Stylonychia ... & EHRBG. & 1830 & mytilus...... & MÜLL. & 177 \\
\hline Tintinnus ... & SCHRANK & 1803 & inquilinus .... & MüLL. & 177 \\
\hline Trachelius .. & SCHRANK & 1803 & 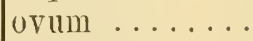 & EHRBG. & 18 \\
\hline odina ... & ЕнRBG. & 1830 & pediculus .. & MÜLL. & \\
\hline Vorticella.... & L. & 1767 & microstoma. & Ehrвя. & \\
\hline Zoothamniem . & EHRBG. & 1838 & arbuscula .... . & EHRBG. & \\
\hline
\end{tabular}

Lit.: BÜtschLI in: Bronn's Kl. Ord., v. 1 1880-1889.

Ehrenberg, Infus., 1838.

\section{Coelenterrita.}

Spongiaria.

\begin{tabular}{|c|c|c|c|c|c|}
\hline Aplysina . . & NaRDo & 1834 & aelophoba & Nardo & 1834 \\
\hline Axinella ...... & О. Аснм. & 1862 & polypoides & О. Sснм. & 1862 \\
\hline Chalina....... & GraNT & 1861 & oculata... & PALL. & 1766 \\
\hline Chondrilla .... & O. Sснм. & 1862 & nucula... & О. Sснм. & 1862 \\
\hline Chondrosia .... & NaRdo & 1833 & reniformis . & NARDO & 1833 \\
\hline Clathria . . . . . & О. Sснм. & 1862 & corallioides. . & Olivi & 2 \\
\hline Cliona........ & Grant & $1826^{\circ}$ & celata .... . & GRANT & \\
\hline Dactylocalyx. . & S'TuchB. & 1841 & pumiceus & Sтиснв. & 18 \\
\hline Emplectella .. & Ow. & 1841 & aspergillum . & Ow. & 1841 \\
\hline Euspongia ... & BRONN & 1859 & officinalis ... & L. & 59 \\
\hline Grantia .... & Flem. & 1828 & compressa & O. $F_{\triangle B R}$. & 178 \\
\hline Halichondria . & FLEM. & 1828 & panicea.... . & PALL. & 176 \\
\hline Halisarca ..... & Dư. & 1838 & dujardinii . . . . & JoHNST. & \\
\hline Hippospongia. . & F. E. Sсн. & 1879 & equina ....... & O. Sснм. & \\
\hline Hyalonema... & GRAY & 1832 & sieboldii & Gray & \\
\hline Lellcandra .... & H. & 1872 & aspera. & O. Sснм. & \\
\hline
\end{tabular}


C. APSTEIN.

\begin{tabular}{|c|c|c|c|c|}
\hline Leuco olenia & BwBK. & |1862 |blanca & М.-М. & 1868 \\
\hline Reniera .... & Nardo & 1833 cratera & O. Sснм. & 1862 \\
\hline Semperella . & GRAY & 1868 schultzei & $G_{R \mathbf{A}}$ & 1868 \\
\hline Spongelia . . & NARDO & 1834 pallescens. & О. Зснм. & 1862 \\
\hline Spongilla & LM. & 1815 lacustris . & L. & 1759 \\
\hline Suberites & Nardo & 1833 domuncula & OuIvI & 1792 \\
\hline Sycon ... & Risso & 1826 raphanus . & О. Sснм. & 1862 \\
\hline Tethya. & LM. & 1815 lyncurium. & L. & 76 \\
\hline
\end{tabular}

Lit.: VosMuer in: Brons's Kl. Ord., v. 21887.

Anthozoa.

Actinia

Adamsia .......

Agaricia

Alcyonium ....

Alveopora ....

Amphelia .

Anemomia.....

Antipathes....

Arachnactis ...

Astrea .......

Astreoides ....

Bolocera

Bunodes ......

Caligorgia

Caryophyllia . .

Cerianthus .... .

Cladocora.....

Corallium .....

Cryptabacia ....

Cyphastraea...

Dendrogyra...

Dendrophyllia .

Desmophyllum .

Diploria ......

Echinopora ...

Edwardsia ....

Euphyllia..

Eusmilia......

Favia .

Flabellum.....

Fungia
L.

L.

LM.

L.

Q. \& $G$.

1767| equina 1840

1801

M.-E.\& Нм

Risso

PaLt.

SARS

LM.

Buainv.

Gosse

Gosse

(GRAY)

Lim.

Chiaje

EhrbG.

LM.

1833 viridis

1801

1830

1855

1854

1870

1801
1758 digitatum .....

E. 1849 oculata

1766 dichotoma ....

1846 albida ........

M.- E. \& Hure.

M.-E. \& HME.

Eнrbe.

Buainv.

EhrBg.

II. $\mathrm{E}$ \& $\mathrm{H}$

1834

1830

1832 membranaceus .

1834 caespitosa ...

1816 rubrum . . . . . .

1849

talpina .......

microphthalma .

cylindrus .....

1834

Lim.

Quatref.

Dana

M.- E. \& Hме.

OKEN

Less.

Lis.
E. 1849

1816 rosularia .....

1842

1846 fimbriata ....

1848 fastigiata ... .

1815 okeni

1831 par

1801 pavoninum ....

\begin{tabular}{|c|c|}
\hline L. & 1766 \\
\hline ELL. \& & $\left.1786^{1 b}\right)$ \\
\hline SoLAND & \\
\hline L. & 1758 \\
\hline Q. \& G. & $\left.1833^{1 \mathrm{~b}}\right)$ \\
\hline L. & $\left.1758^{12}\right)$ \\
\hline M.-E. & 1857 \\
\hline PALL. & 1766 \\
\hline SARS & 1846 \\
\hline PALL. & $\left.1766^{1 a}\right)$ \\
\hline PALL. & $\left.1766^{1 b}\right)$ \\
\hline ELL. \& & $\left.1786^{12}\right)$ \\
\hline SoLAND & \\
\hline SPALANZ. & 1784 \\
\hline L. & $\left(1767^{12}\right)$ \\
\hline L. & 1758 \\
\hline LM. & $\left.1801^{1 b}\right)$ \\
\hline LM. & $\left.1816^{14}\right)$ \\
\hline EнrвG. & $\left.1834^{12}\right)$ \\
\hline L. & $\left.1758^{1 b}\right)$ \\
\hline M.-F.\& HME & $\left.1848^{12}\right)$ \\
\hline LM. & $\left.1816^{12}\right)$ \\
\hline LM. & $\left.1816^{1 \mathrm{a}}\right)$ \\
\hline SPENGL. & $\left.1799^{1 \mathrm{a}}\right)$ \\
\hline PALL. & $\left(1766^{12}\right)$ \\
\hline M.-E. \& HME. & $\left.1857^{12}\right)$ \\
\hline LESS. & $\left.1831^{12}\right)$ \\
\hline L. & $\left(1758^{1 b}\right)^{x}$ \\
\hline
\end{tabular}


Funiculina ....

Galaxea

Goniastrae

Goniopora

Halomitra ....

Heliastraea ...

Herpetolitha.

Hydnophora . . .

Isis

Leiopathes....

Lithophytum . .

Lophelia......

Lophoseris . . . M.-E. \& HмE. 1849

Madracis ..... M.-E. \& HME.

Madrepora .... L.

Meandrina ....

Melitodes

Merulina

Montipora ....

Mycedium

Oculina

Pachyseris ....

Paracyathus ..

Pavonaria ....

Pectinia

Pennatula

Phyllactis

Phymatus.....

Plesiastraea ...

Plumarella ....

Pocillopora ...

Podabacia ....

Porites

Primnoa

Psammocora . .

Pteroeides ....

Renilla

Rhipidogorgia

Sagartia .....

Surcophytum(on)

Schizopathes ..

Seriatopora ...

Stylophora

OKEN

Q. \& G.

DaNA

M.- E.

Eschz.

Fisch.

L.

Gray

ForsK.

Lim.

VERRILL

EHrBg.

Q. \& G.

ОKeN

LM.

KöLL.

OKEN

L.

M.-E.

KuUNz.

Gray

LM.

M.-E.\& Нме.

Lis.

LMx.

DANA

HERKL.

LM.

VAL.

Gosse

1816

LESS.

Brook

(?)

LM.

SchweIgG.
LM. $\quad 1816 \mid q u a d r a n g u l a r i s$

1815

M.-E. \& HME. 1849

1833 pedunculata

1846 pileus

1857

1825 limax

1810 demidovii

1758 hippuris

1842

1775

M.- E. \& Нме. 1849

arboreum .....

prolifera.....

cristata

M.- E. \& Hмw. 1849

1758

1801

1865

1834

1833

1815

1816

M.- E. \& Hме. 1848

1869

1815

1758

1851

1877

M.- E.\& HмE. 1848

1870

1816

1812

1846

1858

1816

1855

1858

| 1838

1889

1816

filigrana .

ocracea.

pulchellus.....

finmarchica ...

maeandrites ...

phosphorea

urvillii

contigua......

griselum

reniformis ....

flabellum .....

sphyrodeta....

glaucum .
PALL.

$1766^{2}$ )

PALL.

$\left.1766^{1 \mathrm{a}}\right)$

Forsk.

Q. \& G.

L.

Esp.

Esp.

Fisch.

L.

$1775^{1 \mathrm{a}}$ )

$1833^{1 \mathrm{~b}}$ )

$1767^{\mathrm{lb}}$ )

$1797^{12}$ )

$1797^{\mathrm{b}}$ )

$\left.1818^{12}\right)$

1758

Forsk.

1775

PALL.

ELL. \&

$\left.1766^{12}\right)$

Soland.

M.- E. \& HME. $1850^{12}$ )

LiI.

Esp.

I.

ELL. \&

$1816^{1 \mathrm{~b}}$ )

Soluand.

Q. \& G.

Pall.

L.

LiI.

Рнше.

SARS

L.

L.

$\left.1791^{12}\right)$

1758

$\left.1786^{12}\right)$

$1833^{1 \mathrm{~b}}$ )

$1766^{1 \mathrm{~b}}$ )

$1758^{1 \mathrm{a}}$ )

$\left.1816^{1^{b}}\right)$

$1842^{12}$ )

$\left.1851^{2}\right)$

$\left.1758^{1 a}\right)$

$1758^{2}$ )

M.- E. \& HME. $1850^{12}$ )

M.- E.

1857

Esp.

$1797^{1 \mathrm{~b}}$ )

PALL.

LM.

$1776^{1^{\mathrm{b}}}$ )

Gunn.

$\left.1816^{1^{b}}\right)$

Esp.

(BOHADSCH)

1763

$1797^{\mathrm{b}}$ )

PALL.

L.

Gosse

$1761^{2}$ )

Q. \& G.

1833

LM.

$\left.1816^{1 \mathrm{~b}}\right)$

Pall. 
Symphyllia.... M.-E. \& HMe. 1818 sinuosa Q. \& G. $\left.1833^{\mathrm{Ia}}\right)$

Tridacophyllia

Tubipora

Turbinaria ....

Umbellula

Veretillum

Xenia ........ SAv in

OKEN

Cuv.

Cur.
Blaivv. 1830 lactuca

L. $\quad 1758$ hemprichi.....

1815 crater .

1798 encrinus .

1798 cynomorium . . .

SAv. in Ly. 1816 umbellata....

Lit.: ${ }^{1)}$ M.-Edwards \& HaIme, Hist. Corall., 1a) $v .21857$; 1b) 0.31860.

2) Kükenthal in: Tierreich, v. 431915.

Döderlein (Straßburg) Madreporaria, KëKenthal (Breslau) Alcyonidae, Pax (Breslau) die übrigen Gruppen.

\section{Hydromedusae.}

\begin{tabular}{|c|c|c|c|c|c|}
\hline Aegina ... . & Eschz. & 1829 & lrosea... . & Eschz. & $\left.1829^{9 b}\right)$ \\
\hline Aeginopsis & Brantt & 1835 & laurentii . & BRANDT & $\left.1835^{8}\right)^{\prime}$ \\
\hline Aequorea. & Pér. \& LsR. & 1809 & forskalea & Pér. \& LSR. & $\left.1809^{\mathrm{s}}\right)$ \\
\hline Aglaura . & Perr. \& LsR. & 1809 & hemistoma & PéR. \& LSR. & $\left.\left(809^{4}\right)^{8}\right)$ \\
\hline Catablema & H. & 1879 & vesicarium & A. AG. & $\left.1862^{4}\right)$ \\
\hline Cladonema & DUj. & 1843 & radiatum. & DuJ. & $\left.\left.1843^{4}\right)^{8}\right)$ \\
\hline & Еschz. & $18 \div 9$ & tetrastyla & Eschz. & $\left.1829^{4}\right)$ \\
\hline & QTRF. & 1842 & dichotoma & QTRF. & $\left.\left(8+2^{4}\right)^{8}\right)$ \\
\hline Eutin & M.'CRADY & 1857 & $\operatorname{mira} . .$. & M. Crady & $\left.\left(857^{4}\right)^{8}\right)$ \\
\hline nia & PÉr. \& LSR. & 1809 & proboscidalis & Fonsк. & $\left.1775^{9 a}\right)$ \\
\hline & Fwкs. & 1882 & papillosum . & VANHÖFFEN & $\left.1902^{s}\right)^{\prime}$ \\
\hline & LESs: & 1843 & crucia & ForSK. & $\left.\left(1775^{4}\right)^{8}\right)$ \\
\hline & LESS. & 1842 & hylla. & Снам. \&Eys. & $\left(1821^{8}\right)$ \\
\hline Ptychogastria & Alum. & 1878 & polaris & ALLim. & $\left.1878^{8}\right)$ \\
\hline alonema. & GeGenb. & 1856 & & Gegenb. & $\left.\left(856^{4}\right)^{8}\right)$ \\
\hline & Less. & 1843 & tubulosa. & SARS & $\left.\left(835^{8}\right)^{6}\right)$ \\
\hline ndella & H. & 1879 & bitentaculata & Q. \& G. & $\left.1833^{8}\right)$ \\
\hline & A. AG. & 1862 & & A. AG. & $\left.\left(862^{4}\right)^{8}\right)$ \\
\hline $\mathrm{Tu}$ & M.'CRADY & 1856 & untricola & II:Crady & $\left.\left(856^{4}\right)^{8}\right)$ \\
\hline & Fonвes & 1846 & stellata. & FoRB. & $\left.1846^{6}\right)$ \\
\hline & GEGENB. & & costata & Gegenb. & $\left.1856^{4}\right)$ \\
\hline
\end{tabular}

\section{Polypen.}

Abietinaria.... Aglaophenia.. Antennularia . Bougainvillea. Campanularia Campanulina . Clava

Clytia

Cordylophora .
Кснр.

Lixx.

LiI.

Less.

LM.

BENED.

Forsk.

Lir.

ALLM.
$188+$
1812
1810 pluma.

1816 antemina

1830 macloviana.

1816 verticillata.

$1847 \mathrm{t}$

1775

1812 johnstoni

1844 lacustris \begin{tabular}{c|l} 
EhribG. & 1834 \\
Pall. & $\left.1766^{1 \mathrm{~b}}\right)$ \\
L. & $\left.1758^{2}\right)$ \\
PaLl. & $\left.1766^{2}\right)$
\end{tabular}

Stv. $\quad 1816$

\begin{tabular}{c|l} 
Q. ¿G . & $\left.1833^{1 \mathrm{a}}\right)$ \\
PALL. & $\left.1766^{1 \mathrm{a}}\right)$ \\
EHRBG. & $1834^{1 \mathrm{~b}}$ \\
PALL. & $\left.1766^{\mathrm{b}}\right)$ \\
L. & $\left.1758^{2}\right)$ \\
PALL. & $\left.1766^{2}\right)$ \\
SAV. & $1816^{2}$
\end{tabular}


Corymorpha ...

Coryne

Eudendrium

Gonothyraea ..

Halecium .....

Hydra

Hydractinia ...

Lafoea .......

Monocaulus

Myriothela

Pasythea

Peunaria

Plumularia....

Podocoryne ...

Schizotricha...

Sertularella

Sertularia.

Silicularia

Thuiaria ......

Tubularia.....
SARS

G̈̈RTN.

EHRbG.

Alim.

OKEN

L.

Bened.

LMx.

Alem.

SARS

DANA

OKEN

LiI.

SARS

Alitim.

GrAY

L.

MeYeN

FLFM.

L.
$1845 \mid$ nutans

1774 pusilla

1832 ramosum .

1864 lovéni.

1815

1758 vulgaris

1841 echinata

1821 dumosa.

1864 imperator .

1850 phrygia

1846

quadridentata

1815 cavolini

1816

1846

1883

1847

1758

1834 rosea

1828 thuja

1758 indivisa
SARS

GäRTN.

L.

Alilim.

L.

PALL.

Fuem.

Flem.

Allim.

O. $\mathrm{F}_{\mathrm{ABR}}$.

ELL. \&

Soland.

Ehrba.

L.

SARS

Alum.

L.

EuL. \&

Soliand.

MEYEN

L.

I. $\left.\left(1835^{7}\right)^{2}\right)$

$\left.1774^{7}\right)^{2}$ )

$\left.1758^{\circ}\right)$

$\left.1859^{7}\right)$

$\left.1758^{7}\right)$

$\left.1766^{7}\right)$

$\left.1828^{7}\right)^{2}$ )

$\left.1820^{7}\right)$

$1885^{3}$ )

$\left.1780^{3}\right)^{7}$ )

1786

$1832^{3}$ )

$\left.1758^{7}\right)$

$\left.1846^{7}\right)^{3}$ )

$1883^{3}$ )

$\left.1758^{2}\right)$

$\left.1786^{7}\right)$

$\left.1834^{5}\right)$

$1758^{7}$ )

$1758^{7}$ )

Acalephae.

Atolla ...... H. $\quad$ 1880|wyvillei

Aurelia ... . . . . Pér. \& LsR. 1809 aurita

Cassiopeia .... Pér \& Lsr.

Cephea ....... Pér. \& LsR.

Charybdea ....

Chrysaora

Costa

1809 andromeda

1809 octostyla

1846 marsupialis

Pér. \& LsR.

1809

$\mathrm{H}$.

1869

hysoscella.

Crambessa

Pér. \& LsR.

1809

mosaica

L. A G.

1862 gaudichaudi

Desmonema

H. J. Cliark

1863

Haliclystus MÜLL.

1776

L. Ag.

1862

auricula.

Mastigias

KöLL.

185:

Pelagia.

Pér. \& LsR.

1809

STEENSTR.

Periphylla

BRANDT

Cuv.

Rhizostoma

1837 hyacinthina

H.

L.

Forsk.

ForsK.

L.

L.

Q. \& G.

L.

Less.

RAтHKE

MÜLL.

LEss.

KöLL.

ForsK.

SteEnstr.

PALI.

MACRI

$\left.\left(1880^{4}\right)^{8}\right)$

$\left.1758^{4}\right)$

$\left.1775^{4}\right)^{8}$ )

$\left.1775^{1}\right)$

$\left.1758^{4}\right)$

$\left.1766^{9}\right)$

1838 frondosa ......

L. Ag.

1799 pulmo

L. $A G$.

$\left.\left.1824^{4}\right)^{8}\right)$

$1758^{4}$ )

$\left.1829^{4}\right)$

$\left.18\left(16^{4}\right)^{8}\right)$

$\left.\left(1776^{4}\right)^{8}\right)$

$18294)^{8}$ )

$\left.\left.1853^{4}\right)^{3}\right)$

$\left.\left.1775^{4}\right)^{8}\right)$

$\left.\left.1837^{4}\right)^{8}\right)$

$1774^{4}$ )

1778

1862 meleag'is

+1862 .

Lit.: 1) L. Agassiz, Contr. nat. Hist. Amer.
$\left.{ }^{2}\right)$ Allman, Monogr. Hydroids, 1871.

s) Allian, in: Rep. Voy. Challenger, v. 7 1883, v. 231888.

4) Haecket in: Rep. Voy. Chaldexger, v. 18 pars 401887. 
Lit.: $\left.{ }^{8}\right)$ Hartlaub in: Zool. Jahrb., suppl. 61905 (Fauna Chil.)

6) B

7) Hincks, Hist. Brit. Hydr., 1868.

3) A. G. ILAYER, Medusae World, v. 1-3 1910 (Publ. Carnegie Inst.).

9 a b) V $\triangle$ NHÖFFEN in: Ergeb. Tiefsee-Exp., v. 3 1902, v. 191908.

VANHöfFen (Berlin), Hartlaub (Helgoland)

Milleporidae.

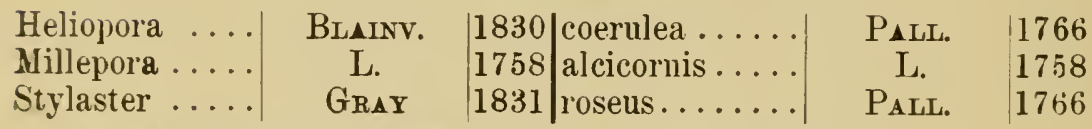

Lit: IL.-Edwards \& Haime, Hist. Corall., v. 2,3 1857, 1860.

DöDERLeiN (StraBburg).

Siphonophorae.

Abyla .

Abylopsis $\ldots .$.

Apolemia .....

Diphyes

Galeolaria.....

Hippopodius...

Monophyes ...

Nectalia ......

Physalia .....

Physophora ...

Porpita.......

Praya .........

Velella
Q.\& G.

Chun

Eschz.

Cuv.

LsR.

Q. \& G.

Claus

$\mathrm{H}$.

LM.

Forsk.

LM.

Blainv.

LM.
182? |trigona .......

1888 pentagona ....

1829

1817

1807 quadriva

1807 quadrivalvis ...

1827 luteus ........

1873

1888

1801 arethusa .....

1775

1801 hydrostatica..

1834

1801
Q. \& G.

Q. \& G.

LsR.

C Cok. 1807 )

$\left.1827^{2}\right)$

$\left.1827^{2}\right)$

$1807^{3}$ )

Снцм. \&Ers. $1821^{2}$ )

LsR.

Q. \& G.

Claus

$\mathrm{H}$.

Browne

Fonsk.

MÜLL.

Chiaje

Forsk. $\left.1807^{2}\right)$

$1827^{3}$ )

$1873^{2}$ )

$\left.1888^{3}\right)$

$\left.(1756)^{3}\right)$

$1775^{3}$ )

$1776^{3}$ )

$1842^{2}$ )

$1775^{3}$ )

\section{Ctenophorae.}

Beroe ....... Browne $\mid$ (1756)|cucumis .....

Bolina ...... Mert. 1833 infundibulum ..

Callianira ..... PÉr. \& LsR. 18

Cestus....... LsR.

Eucharis ..... Eschz.

Hormiphora ... L. AG.

Mertensia .... Less.

Pleurobrachia . FLEM.

\begin{tabular}{c|c} 
O. FABR. & $\left.1780^{3}\right)$ \\
O. F F & $\left.1780^{3}\right)$ \\
CHIAJE & $\left.1841^{1}\right)$ \\
LsR. & $\left.1813^{1}\right)$ \\
Q. \& G. & $\left.1824^{1}\right)$ \\
SARS & $\left.1856^{1}\right)$ \\
O. FABR. & $\left.1780^{3}\right)$ \\
O. FABR. & $\left.1780^{3}\right)$
\end{tabular}

Lit.: 1) Chun in: Fauna Flora Neapel, v. 11880.

2) Hoppe-Moser in: D. Südp.-Exp., 1916.

3) VАKHöfFeN in: Nord. Plankton, v. 6 1903, 1906. 


\section{Echinodermata.}

Crinoidea.

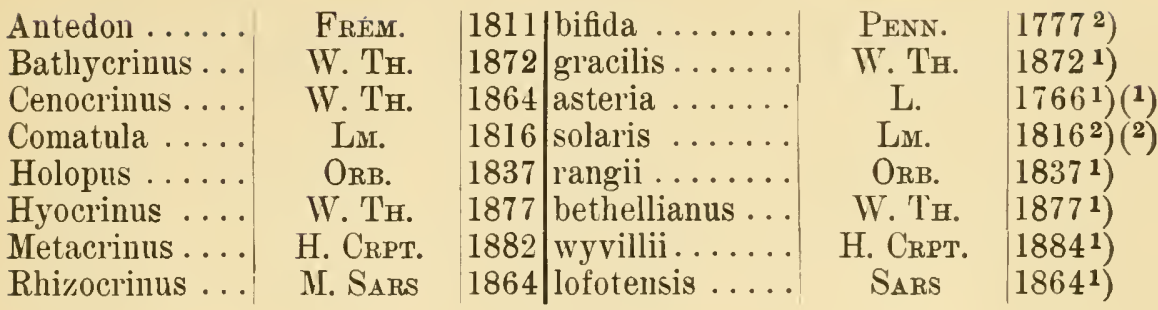

Asteroidea.

Acanthaster...| Gerv. $\quad 1841 \mid$ echinites...... ELL. \&

Anthenea.... Gray 1840 pentagonula..

Archaster..... J. MüLL.\& 1840 typicus . .

Solund. Trosch.

Asterias ......

L.

1758 rubens

Asterina

L. AG.

Asterodiscus .. GRAY

1835 gibbosa.

Astropecten ...

GraY

Chaetaster .... J. MÜLL. \&

1847 elegans Trosch.

Ctenodiscus ... J. MÜLL. \& 1842 crispatus .... 1840 aurantiacus 1840 longipes Trosch.

Culcita .

L. Ag.

Echinaster .... J. MÜLL. \&

1835 schmideliana . . Trosch.

Fromia

GRAY

Goniaster..... L. AG.

Goniodiscus ... J. MÜLL. \& Trosch.

Gymnasteria .

GRAY

GRAY

Henricia..... G GRAY

Hippasteria

GRAY

Liuckia.

NARDO

Luidia

Forb.

Nardoa.

GRAY

Nectria...... GRAY

Nidorellia..... GRAY

Ophidiaster ... L. AG.

1840 sepositus

LM.

$\left.1786^{3}\right)$

18164)

J. MÜLL. \& 1840 4)

Trosch.

L.

$\left.1758^{3}\right)$

Penn.

GraY

$\left.1774^{4}\right)$

L.

RETZ.

$\left.1847^{4}\right)$

$\left.1758^{3}\right)\left({ }^{3}\right)$

$\left.1805^{4}\right)$

Retz.

$\left.1805^{4}\right)$

RETZ. $1805^{4}$ )

RETZ.

$1783^{3}$ )

LM.

$\left.1816^{3}\right)$

LM.

LM.

$\left.1816^{4}\right)(4)$

1842 pleyadella

$1816^{4}$ )

LM.

$\left.1816^{4}\right)$

LM.

$\left.1815^{3}\right)$

MÜLL.

PAReL.

GM.

AUD.

RETZ.

$\left.1776^{3}\right)(5)$

1840 sanguinolenta.

1834 laevigata

1039 savignyi

1840 variolata

1840 ocellata

E. PERR.

$\left.1770^{4}\right)$

GRAY

LM.

$\left.1788^{3}\right)\left({ }^{6}\right)$

$1809^{4}$ )

1840 armata .......

1835 ophidianus

$1805^{3}$ )

$1876^{4}$ )

$\left.1840^{4}\right)$

$1816^{3}$ )

Anm.: 1) sub Pentacrinus a., 2) sub Actinometra 8., 8) sub A. aranciacus, 4) sub Pentagonaster semilunatus, ${ }^{5}$ ) sub Cribrella oculata, ${ }^{6}$ ) sub L. miliaris. 


\begin{tabular}{|c|c|c|c|c|c|}
\hline Oreaster... & $\begin{array}{c}\text { J. MÜLL. \& } \\
\text { TROSCH. }\end{array}$ & 1842 & |mamillatus . & AUd. & $\left.1824^{4}\right)$ \\
\hline Palmipes .... & L. AG. & 1835 & membranaceus & RETZ. & $\left.1783^{4}\right)$ \\
\hline Pentagonaster & $G_{R A Y}$ & 1840 & pulchellus.... & GRAY & $\left.1840^{4}\right)$ \\
\hline Pteraster.... & $\begin{array}{l}\text { J. MÜLL. \& } \\
\text { TROSCH. }\end{array}$ & 1842 & militaris ... & MÜLL. & $\left.1776^{4}\right)$ \\
\hline Solaster & Forb. & 1833 & endeca. & Retz. & $\left.1783^{3}\right)$ \\
\hline Stellaster & GRAY & 1840 & equestris & RETZ. & $\left.1820^{4}\right)$ \\
\hline Stichaster & $\begin{array}{l}\text { J. MÜLL. \& } \\
\text { Trosch. }\end{array}$ & 1842 & roseus... & MÜLL. & $\left.1788^{3}\right)$ \\
\hline Tosia & GRAY & 1840 & australis . & $\mathrm{GRAY}_{\mathrm{RA}}$ & $\left.1840^{4}\right)$ \\
\hline
\end{tabular}

\section{Ophiuroidea (Lit. ó).}

Amphiura....| Forb. $|1842|$ filiformis ....| MüLL. $\mid 1776$ Asteronyx .... J. MÜLL. \& 1842 loveni ........ J. MÜLL. \& 1842

Trosch.

Astrocladus ... VerRILL 1899 enryale.

Astrophyton... J. MÜLL. \& 1842 muricatum .... Trosch.

Astroschema .. Ö̈sт.\&LÜтк. 1856 oligactes . . . . . .

Euryale ...... Lir.

Gorgono-

cephalus....

Hemipholis....

Ophiacantha ...J.1

Ophiactis .....

Ophiarachna

Ophiarthrum . .

Ophiocnemis

Ophiocnida... .

Ophiocoma....

Ophiocten

Ophioglypha ..

\section{0 0} 0

\section{LEACH}

LYм.

J. MÜLL. \& 'Trosch.

LÜтк.

J. MÜLL. \& Trosch.

\section{Peters}

J. MÜLL. \& Trosch.

LyM.

L. AG.

LÜTK.

LYMr.

J. MÜLL. \& Trosch.

J. MÜLL. \& Trosch.

J. MÜLL. \&

\section{'Trosch.}

LÜTK.
1816

aspera $\ldots . . .$.

1815

1865 cordifera .....

$18+2$
Trosch.

RETz.

Lir.

Pall.

Ly.

L.

Bosc $\quad 1830$

RETZ. 1805

$1783\left(7^{7}\right)$

$1816\left(^{\circ}\right)$

1788

1816

$1761\left({ }^{9}\right)$

bidentata..... RETz.

\begin{tabular}{l|l}
1856 & savignyi .... . . \\
1842 & incrassata ... \\
1851 & elegans ....... \\
1842 & marmorata . . .
\end{tabular}

1865

1835

1854 sericeum......

1860 ciliata........

1840 annulosa.......

J. M

\begin{tabular}{l|l} 
MÜLL. \& & 1 \\
TrOSCH. \\
LM.
\end{tabular}

1842

1816

Peters

1851

Liv.

1816

Mont. 1804

Lir. $\quad 1816$

Forb. 18.52

RETZ. 1805

Blainv. 1834

LiI. $\quad 1816$

Lx.

1816

SAY 


\begin{tabular}{|c|c|c|c|c|c|}
\hline Ophiopholis ... & J. MÜLL. \& & 1842 & |aculeata..... & Retz. & 1783 \\
\hline Ophioplocus . . . & LYM. & 1861 & imbricatus & J. MÜLL. \& & 1842 \\
\hline Ophioscolex & $\begin{array}{c}\text { J. MÜLL. \& } \\
\text { TROSCH. }\end{array}$ & 1842 & glacialis & $\begin{array}{l}\text { Trosch. } \\
\text { J. MÜLL. \& } \\
\text { Trosch. }\end{array}$ & 1842 \\
\hline Ophiotliela. & VERRUL & 1867 & mirabilis . . & VERRILL & 1867 \\
\hline Ophiotlirix & $\begin{array}{l}\text { J. MÜLL. \& } \\
\text { TROSCH. }\end{array}$ & 1842 & flagilis ... & $\begin{array}{l}\text { ABILDG. in } \\
\text { AIÜLL. }\end{array}$ & 1789 \\
\hline Ophimra .... & LM. & 1801 & lacertosa .... & LM. & $1801\left(^{10}\right)$ \\
\hline Tricaster . . . . . & L. AG. & 1835 & |palmifer ...... & Lar. & 1816 \\
\hline
\end{tabular}

Echinoidea (Lit. 6).

Agassizia

Amblypneustes Arachnoides ... Arbacia Asthenosoma . Astriclypelıs . . . Astropyga .... Breyuia

Brissopsis

Brissus

Centro-

stephanus...

Cidaris . . . . . .

Colobocentrotus

Diadema .....

Echinarachnius

Echinocardium

Echinocyamus

Echinodiscus .

Echinolampas

Echinometra .

Echinoneus ...

Echinothrix ...

Echinus

Encope

Fibularia

Goniocidaris

Heterocentrotus

Laganum
TAL.

L. $A G$.

L. AG.

GraY

Grube

VERRILL

Gray

DES.

L. Ag.

LESKE

Peters

LESKE

BRaNdT

Gray

LESKE

Gray

LESKE

LESKE

Gray

GraY

LESKE

Peters

L.

L. $A G$. Lis.

L. Ag.

BrandT

L. $A G$.
|1846| scrobiculata ...

1841

1841

1835

1867

1867

1825

1847 australasiae ...

1840

1778

lacenta......

ixula

varium .

manni .

adiata

lyrifer .......

unicolor

1855 longispinus . . .

1778 papillata. . . . .

1835 mertensii

1825 setosum .....

1778 parma.......

1825 cordatum .....

1778 pusillus ......

1778 auritus .......

1825 oviformis .....

1825 lucunter ......

1778 cyclostomus ...

1853 calamaris ....

1758 esculentus ....

1841 emarginata...

1816 ovulum .......

1846 geranioides ...

1835 mamillatus ....

1841 laganum .
Tal.
Blainv.
L.
L.

Grobe

VERRILL

LESKE

LEACH

Forb.

LESKE

Phil.

Leske

BRANDT

LESKE

Liv.

Penn.

MÜLL.

LESKE

GM.

L.

LESKE

Pall.

L.

LESKE

GM.

LM.

L.

LESKE
1846

1825

1758

$1758(\mathbf{1 1})$

1867

1867

1778

1815

1841

1778

1845

$1778(12)$

1835

1778

1816

1777

1776

1778

1788

$1758(18)$

1778

1774

1758

1778

1788

$1816^{\circ}$

1758

$1778(14)$

Anm.: ${ }^{10}$ ) sub O. laevis, 11) sub A. pustulosa, 19) sub Dorocidaris p., 13) sub E. subangularis, 14) sub L. bonani. 


\begin{tabular}{|c|c|c|c|c|c|}
\hline sovenia ... & L. AG. & $18+7$ & |elongata & GRAY & 1845 \\
\hline Loxechinus & DES. & 1856 & albus... & MoL. & $1782\left({ }^{(15}\right)$ \\
\hline Maretia ... & Gray & 1855 & planulata & Lu. & 1816 \\
\hline Mellita & L. AG. & 1841 & testudinata. & L. AG. & 1841 \\
\hline Leoma. & GRAY & 1851 & & GRAY & 1851 \\
\hline Mespilia & L. AG. \& DES. & 1846 & globulus & L. & 1758 \\
\hline eta & $G_{R A Y}$ & 1855 & sternalis & Lx. & 1816 \\
\hline phus & L. AG.\& DES. & & atus. & L. AG.\&DES. & 1846 \\
\hline Ioer & MIChLN. & 185.5 & & M ICHLN. & $1855\left({ }^{16}\right)$ \\
\hline trotus & Mortsn. & 1903 & lividu & LM. & $1816(17)$ \\
\hline & A. AG. & 1863 & & A. AG. & 1863 \\
\hline hyllacanthus & BraNDT & 1835 & & Lu. & 1816 \\
\hline 00 & L. AG. & 1840 & & L. & $17.58(18)$ \\
\hline & DEs. & 1854 & $\mathrm{pl}$ & W. Тн. & 1869 \\
\hline our & A. AG. & 1869 & $\operatorname{mir}$ & A. AG. & 1869 \\
\hline & L. AG. \& DES. & 1846 & mil & Gм. & $1788(19)$ \\
\hline & L. AG. & 1841 & & LESKE & $1778(20)$ \\
\hline ll & L. AG. & 1841 & bicolor & L. AG. & 1841 \\
\hline & L. AG. & 1847 & & L. & $1758(21)$ \\
\hline & LESKE & 1778 & & LESKE & 1778 \\
\hline oneustes & L. AG. & 1841 & variolaris & Ln. & 1816 \\
\hline & $B_{B A}+{ }_{1}$ & & & & 1776 \\
\hline urus & & & & LESKE & 1778 \\
\hline & L. A \& \& Des. & 1846 & & MoL. & $1782\left({ }^{22}\right)$ \\
\hline & L. AG. & & & LM. & 1816 \\
\hline & & & escuintus & LESKE & $1778\left({ }^{23}\right)$ \\
\hline
\end{tabular}

Holothurioidea (Lit. 7).

Chiridota .....

Cucumaria

Holothuria ....

Phyllophorus..

Psolus.

Stichopus .....

Synapta

Thyone.
Eschz.

Blainv.

GunN.

GRCBE

OKEN

BrandT

Eschz.

OkEN
1829 |laevis

1830 frondosa

$17 ; 57$ tubulosa

1840

1815

1835

pir

1829 inhaerens .

181 ) fusus .
O. FARB.

GCxw.

GiI.

Grube

Strutssf.

Brandt

MÜLL.

MIÜLL.
1780

1770

1791

1840

1865

1835

$1770^{\circ}$

1788

Lit.: 1) Carpenter in: Rep. Voy. Challenger, v. 11 pars 321884.

2) Carpenter in: Rep. Voy. Challenoer, v. 26 pars 601888.

3) E. Perrier in: Areh. Zool. expér., v. 41875.

4) E. Perrier in: Arch. Zool. expér., v. 51876.

5) Lymax in: Rep. Voy. Challenger, v. 5 pars 141882.

6) A. Agassiz, Revis. Echini 1872.

7) Lampert in: C. Sfmper, Reis. Philipp., v. 4 Abt. 31885.

DöDERLEIN (StraBburg).

Anm.: 15) sub Strongylocentrotus a., 16) sub Moira a., 17) sub Strongylocentrotus b., 18) sub Colobocentrotus a., 19) sub Echinus m.. 20) sub R. rumphii, ${ }^{21}$ ) sub S. canaliferus, $\left.{ }^{22}\right)$ sub Arbacia n., ${ }^{23}$ ) sub Hipponoe e. 


\section{Vermes.}

\section{Mionelminthes.}

\begin{tabular}{l|c|c|c|c|c} 
Dicyema...... & KöLL. & 1849 & typus ........ & BENED. & 1876 \\
Rhopalura .... & GIARD & 1879 & ophiocomae ... & GLARD & 1879 \\
Trichoplax .... & F. E. Sch. & 1883 & adhaerens.... & F. E. SCH. & 1883
\end{tabular}

Lit.: BraUn in: Bronn's Kl. Ord., v. 4 I 1894-1900.

Cestodes.

Acanthobothrium .... Bened.

Amphilina .... W WENR.

Anthobothrium

BENed.

Bothridium ... BuaINV.

Bothriocephalus

Brems.

Calliobothrium .

Bened.

Caryophyllaeus

Gyrocotyle ....

Ligula

Phyllobothrium

Schistocephalus

Taenia .......

Tetrarhynchus .

Gis.

DIES.

Bu.

Bened.

Crepl.

L.

Rud. \begin{tabular}{|l|l|}
1850 & coronatum . . . . \\
1858 & foliacea . . . . . \\
1850 & cornucopiae . . \\
1824 & pythonis . . . . \\
1819 & latis . . . . . . \\
1850 & verticillatum . . \\
1790 & mutabilis . . . . \\
1850 & rugosa . . . . . \\
1782 & avium . . . . . \\
1849 & lactuca . . . . . \\
1829 & nodosus . . . . . \\
1758 & solium . . . . . . \\
1809 & attenuatus . . .
\end{tabular}
RUD.

RUD.

BENED.

Blainv.

BREMS.

RUD.

RUD.

Dies.

BL.

Bened.

BL.

L.

Ruv. $\left.1819^{1}\right)$

$\left.1819^{1}\right)$

$1850^{1}$ )

$1824^{1}$ )

$\left.1819^{2}\right)$

$\left.1819^{\prime}\right)$

$\left.1802^{1}\right)$

$1850^{1}$ )

$1782^{1}$ )

$1850^{1}$ )

$1782^{1}$ )

$\left.1758^{1}\right)$

$1819^{3}$ )

Lit.: 1) Braun in: Brons's Kl. Ord., v. 4 I 1894-1900.

2) Ledckart, Paras., v. 1, 2 1863-1876.

${ }^{3}$ ) Diesing. Syst. Helm., v. 1, 2 1850, 1851.

BRAUN (Königsberg), Linstow (Göttingen).

\section{Trematodes.}

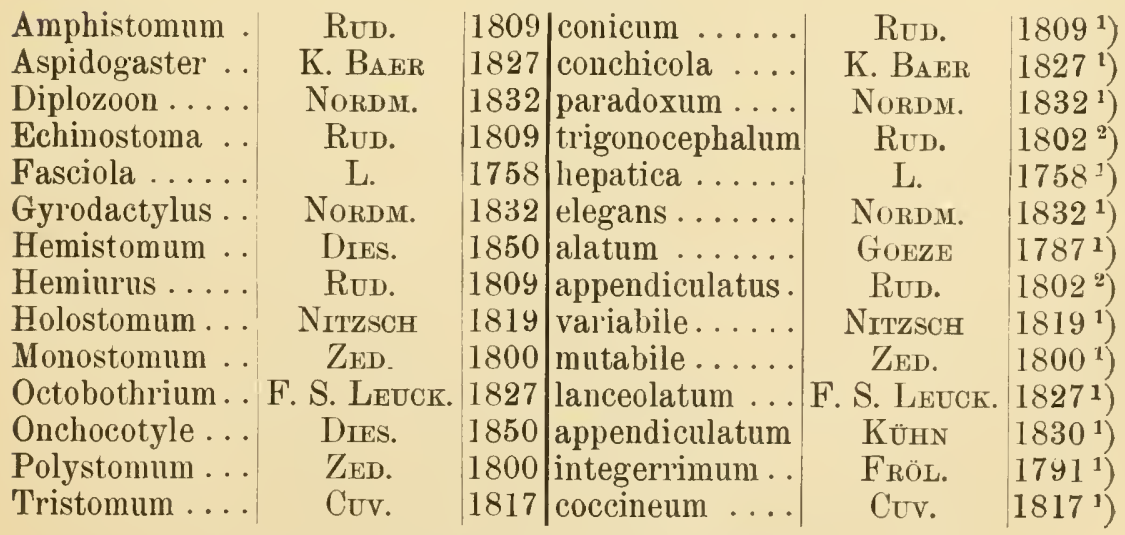

Lit.: 1) Braun in: Bronn's K1. Ord., v. $4^{1} 1894-1900$.

2) Rudolphi, Entoz. Synops., 1819. 


\section{Turbellaria.}

Alaurina.....

Allostomum

Aphanostoma.

Bdelloura .

Bipalium

Catenula

Cercyra

Convoluta .....

Dalyellia

Dendrocoelum

Diplonchus... .

Discocelis.....

Eurylepta.....

Geobia

Geoplana .

Gyratrix

Haplodiscus

Leptoplana.

Macrostomum .

Mesostoma... .

Microstomum . .

Monocelis .....

Oligocladus ...

Opistomum ....

Otocelis

Phaenocora

Plagiostomum

Planaria .

Planocera.....

Polycelis

Polycladus

Procerodes .

Promesostoma.

Prorhynchus .

Provortex .

Psendoceros.

Rhynchodesmus

Stenostomum . .

Stylochus

Thysanozoon ..
W. Busch

Bexed.

ÖRsT.

LEIDY

STIMPS.

Dug.

O. Schis.

ÖRST.

FLen.

ÖRst.

StIIIIPS.

EнRBG.

EhrbG.

DiEs.

Fr. MÜLL.

EhrbG.

WELDON

Ehrba.

O. SсHм.

EнrBg.

О. Гснм.

EhrbG.

LANG

O. Sснм.

DiEs.

Ehrba.

О. ЗСНм.

MÜLL.

BLanNv.

EHrbG.

BLANCH.

Girard

GrafF

II. Schultze

GRAFF

LANG

LEIDY

О. Аснм.

EhrbG.

Grube
1851 prolifera

1861 pallidum .

1845 griseum

1851 candida

1858 marginatum .

1832 lemuae

1862 hastata.

1843 paradoxa

1822 viridis

$18+4$

1857

1832

1831 colmuta.

1861 subterranea

1861 subterranea

1831 liel'maphroditus

1888 piger

1831 tremellaris ....

$18+8$ appendiculatum

1837 ehrenbergi ....

1848 lineare

1831 lineata

1884 sanguinolentus .

1848 pallidum .

1862 rubropunctata

1837 mipunctata . .

1852 girardi .......

1776

1828

1831

1847

1850 wheatlandi ....

1882 marmoratum ..

1851 stagnalis

1882 balticus

1884 aurantiaca ....

1851 terrestris

1848 leucops.

1831 neapolitanus

$1840 \mid$ brocchii
IV. Busch

Bened.

ÖRST.

Girard

LOMANN

Dug.

O. Sснм.

ÖRST.

SHaw

MÜLL.

Struips.

BLANCH.

EhrbG.

Fr. MÜLL.

Fr. MÜLL.

EhrbG.

WELDON

ÖRsT.

O. FABR.

Focke

MÜLL.

IIÜLL.

QUATRF.

O. Sсна.

O. Sснат. ÖRsT.

O. Sсни.

MÜLL.

LANG

FR. МÜLL.

Blaxch.

Gikard

M. Schultze 18511)

M. SchuLTZE $1851^{1}$ )

M. Schultze $1851^{1}$ )

СНцале

IÜLL.

DUt.

Chlaje

Grube
1851 1 )

18611)

$\left.1845^{2}\right)$

18524)

$\left.1888^{5}\right)$

18321)

$\left.1862^{4}\right)$

$\left.18+3^{2}\right)$

17911)

$1776^{3}$ )

$\left.1857^{6}\right)$

$1847^{6}$ )

$1831^{6}$ )

$\left.1857^{5}\right)$

$\left.1857^{5}\right)$

$\left.1831^{1}\right)$

$\left.1888^{2}\right)$

$1843^{6}$ )

$\left.1826^{1}\right)$

$\left.1836^{1}\right)$

$\left.1774^{1}\right)$

$\left.1774^{1}\right)$

$1845^{6}$ )

$\left.18+8^{1}\right)$

$1852^{2}$ )

184.31)

$\left.1857^{1}\right)$

$\left.1776^{3}\right)$

$1879^{6}$ )

$1831^{3}$ )

$\left.1847^{5}\right)$

$\left.1850^{4}\right)$

$1822^{6}$ )

$1774^{5}$ )

1828 1 )

$18+1^{6}$ )

$1840^{6}$ )

Lit.: 1) GrafF in: Tierreich, v. 351913.

2) Graff in: Tierreich, v. 231905.

3) Steinmann in: Monogr. einheim. Tiere, v. 51913.

4) Wilhelmi in: Fauna Flora Neapel, v. 32 1909.

5) GrafF, Monogr. Turb. II., Tricl. terric., 1899.

6) LaNG in: Fauna Flora Neapel, v. 111884.

Steinmaní (Aarau). 
Nemertini.

Amphiporus ... EHrbG. 1831 |lactifloreus... o|

Cephalothrix .

ÖRST.

1843

JoHNST.

828

$\begin{array}{ll}\text { J. RATHKE: } & 799\end{array}$

Cerebratulus

REN.

$180+$ marginatus ....

Lineus ....... J. Sow.

1806

REN.

804

Malacobdella .. BLAinv.

1827 grossa ........

GunN.

MÜLL.

1770

1776

Lit.: Bürger in: Tierreich, v. 201904.

Nematodes.

Ancylostoma*).

Angiostoma ...

Anguillula ....

Ascaris

Cucullanus ....

Dorylaimus ...

Dracunculus...

Eustrongylus . .

Filaria .......

Gordius

Heterakis .....

Heterodera....

Mermis .......

Oncholaimus ..

Oxyluris .....

Rhabditis ....

Sclerostomum

Strongyloides .

Strongylus ....

Syngamus .....

Trichina ......

Trichocephalus

Trichosoma ...

Tylenchus ....
DUBINT

DUJ.

MÜLI.

L.

MÜLL.

DUJ.

ReICHARD

DIES.

MÜLL.

L.

DUJ.

AD. Sснм.

DUJ.

DUJ.

RธD.

DUJ.

RUD.

Grassi

MÜLI.

SIEB.

Ow.

Goeze

RUD.

Bastian
1843 d duodenale.....

1845 nigrovenosum . .

1786

1758

$\left\{\begin{array}{l}(1774 ?) \\ 1777\end{array}\right\}$

1845

1759

1851

1787

1758

1845

1871

1842

1845

ceti .

umbricoides ..

elegans

stagnalis .....

medinensis ....

gigas .......

martis.

1803

1845

1809

1879

aquaticus .

inflexa

schachti

igiescens

vulgaris .

vermicularis...

terricola ......

1780

1836

1835

1782

1819

equinum

1865
DormTI

ZED.

MÜLL.

L.

ZED.

DUJ.

L.

RUD.

GM.

L.

ZED.

AD. ScHM.

DUJ.

Bastian

L.

DUง.

MÜLL.

Bavay

Sieb.

Ow.

RUD.

.DUJ.

BAUER $\left.1843^{1}\right)$

$\left.1800^{7}\right)$

$\left.1786^{8}\right)$

17581)

$1800^{10}$ )

$1845^{2}$ )

$\left.1758^{6}\right)$

$\left.1802^{1}\right)$

$\left.1791^{11}\right)$

$1758^{5}$ )

$1800^{10}$ )

$\left.1871^{13}\right)$

18424)

$1865^{2}$ )

$\left.1758^{1}\right)$

$\left.1845^{2}\right)$

1780

1876 1 )

$\left.1836^{9}\right)$

$\left.1835^{6}\right)$

$\left.1801^{6}\right)$

$\left.1845^{12}\right)$

$\left.1823^{2}\right)$

Acanthocephali.

Echinorhyn-

[Zӧ̈GA] chus ...... MÜLL.

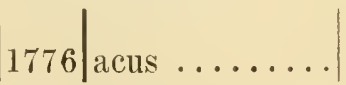

RUD.

$\left.1802^{3}\right)$

Lit.: 1) Braun-Setfert, Tier. Paras. Mensch., ed. 41908.

2) Bastian, Anguill., 1865.

3) Diesing, Syst. Helm., v. 1, 2 1850, 1851.

4) Hagmeifir in: Zool. Jahrb. Syst., v. 32 1912.

5) Meissner in: Z. wiss. Zool., v. 71855 p. 57.

6) R. Leuckart, Paras., v. 21876.

*) Agchylostoma. 
Lit.: `) Linstow in: Arch. Naturg., v. 521 1886, v. 5 hI 1890.

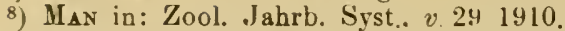

9) Railliet, Traité Znol., ed. 2 Paris 1893.

10) Ant. Schneider, Mlunogr. Nemat., 186i.

11) Stossich, Filarie e Spir., 1897.

12) Sтossich, Trichosoma, 1890.

13) Strubell in: Bibl. Zool., fasc. 21888.

Collin (Berlin), Menzel (Basel).

\section{Chaetognathi.}

Sagitta.....| Q. \& G. |1827|bipunctata....| Q. \& G. 1827 Lit.: Ritter-Zahoyy in: Tierreich, v. 291911.

\section{Archiannelides.}

Polygordius ... $\quad$ Schn. $\mid$ I868|lacteus ...... Rathкe $\mid$ R 483 Lit.: Frarpont in: Fauna Flora Neapel, v. 141887.

\section{Polychaeta.}

Acholoe ......

Alciopa.......

Ampharete....

Amphinome ...

Amphitrite....

Aphrodita

Arenicola .....

Aricia

Audoninia

Autolytus .....

Branchiomma. .

Capitella......

Chaetopterus .

Chloëia

Cirratulus.....

Clymene ......

Dasybranchus

Diopatra.

Ephesia

Clap.

1870 AUD. \& M.-E. 1833 cantrainii MALMGR.

1865

BRUG.

MÜLL.

L.

Lx.

SAv.

QuATREF.

1789 rostrata

Grube

KöLL.

BlaiNV.

Cuv.

LNI.

LN.

1771 cirrata

1758 aculeata

1801 marina

1820 cuvieri

Chuaje

Снiaje

18.2. 1 )

MaLMgR.

$\left.18+1^{1}\right)$

PaLL.

$\left.1 \leqslant 65^{4^{a}}\right)$

MÜLL.

1766

L.

L.

$\left.17884^{a}\right)$

$17581)$

1767

Aud. \& MI.-E.! 18:3+ ${ }^{3 \mathrm{~b}}$ )

Mont. $1815^{3 \mathrm{~b}}$ )

1851 prolifer . . . . . .

MÜLL.

$\left.1788^{10 a}\right)$

Mont.

$\left.1815^{3 \mathrm{~b}}\right)$

O. FABR.

$\left.1780^{6}\right)$

ReN.

$\left.1807^{3 \mathrm{~b}}\right)$

PALl.

$1766 ;$

MÜLL.

$\left.17764^{\mathrm{b}}\right)$

Sav. in Lur. 1818 lumbricoides...

QUATREF.

Grube

1851 caducus

Aud. \& M.-E.

Eteone.

Rathke

SAY.

Euchone.

MaLilgr.

ÖRsT.

Cov.

Eunice

1829

Grube

Clap.

Rathie

1843 gracilis . . . . . .

O. FABR.

$18\left(65^{3 \mathrm{~b}}\right)$

$\left.1851^{6}\right)$

$\left.186.5^{5}\right)$

1822

Hava .........

I86i) papillosa.....

SARS

$18+3^{3 \mathrm{c}}$ )

MÜLL.

$\left.1,80^{4 \mathrm{a}}\right)$

Euphrosyne ... $\mathrm{S}_{A \mathrm{v}}$.: in Lar.

Exogone

Örst.

Fabricia

Buatev.

$184: 3$

1817 nolvegica L.

SAv.

$\left.1850^{4 a}\right)$

Filigrana

OKEN

SARS

18tj naidina.

1828 sabella

ÖRsT.

$177\left(6^{4 a}\right)$

Flabelligera

1815 implexa

Ehrig.

BERK.

$17\left(i 66^{5}\right)$

Отто

1818

1829 diplochaetus

(4⿻15

$\left.1836^{3 \mathrm{~b}}\right)$

$1827^{\mathrm{sb}}$ )

1836 
Glycera ...... SAv. in Lu. $1818 /$ capitata . Aud. \& M.-E. 18:33 maculata

Goniada .

A. Costa

Halla

KinB.

1844 parthenopeia

Harmothoe... .

Helmione .....

Hesione

BLAINV.

Heterocirrus

SAv. in Lar.

1856 imbricata

Hydroides

Lagis

Lanice

Lepidonotus ...

Lopadorhyn-

chus

Lumbriconereis

Lysidice ......

Maldane

Grube

Guns.

Malmgr.

Maliggr.

LEACH

Myrianida

Myxicola

Nephthys

Nereis.

Nerine

Notomastus

Notophyllum

Odontosyllis ..

Onuphis

1828 hystrix

1818 splendida

1855 saxicola

1768 uncinata.

1865 koreni.

GRUBe

Blainv.

SAv, in LM.

GruBe

M.-E.

H. KoCH

Cuv.

L.

1865 conchilega

1816 squamatus

1855 brevis

GRI $B E$

$1855^{9}$ )

1828 fragilis

MÜLL.

$\left.1788^{5}\right)$

Aud. \& M.-E. $1836^{5}$ )

Grube

Mont.

$\left.1860^{4 a}\right)$

ReN.

O. FABR.

$1808^{8}$ )

1847 infundibulum

$1804^{3 \mathrm{~d}}$ )

JoHNST.

1758 pelagica

L.

$\left.1780^{5}\right)$

SARS

ÖRsT.

ClatP.

1838 vulgaris

JoHNST.

$\left.1758^{5}\right)$

SARS

SARS

$\left.1828^{8}\right)$

Clap.

$1850^{6}$ )

1843 foliosum

AUD.\& M.-E.

1863 gibba

Ophelia

SAV.

ÖRst.

182.2 limacina

SARS

Oplrelina.

SARS

Lir.

Pectinaria

GREEFF

JoHNST.

Pholoe

Phyllochae-

topterus

Phyllodoce....

Pista

Grube

Sav. in La.

Maliigr.

Placostegus

PHIL.

$\mathrm{Bosc}$

Polymmia

MaLifrer.

1843 acuminata

RATHKE

Örst.

$183{ }^{\circ}$

ChIAJE

MÜLL.

GREEFF

O. FABR.

$\left.1863^{10 a}\right)$

$1833^{2}$ )

$\left.1843^{4 b}\right)$

$18+3$

181s auricoma

$1825^{3 a}$ )

1879 pictus

$\left.1776^{4 a}\right)$

$\left.1879^{\circ}\right)$

$\left.1780^{4}\right)$

Polltodora

GreefF

Rissos

L.

Lis.

Sabellaria

M.- E.

1839

minuta

Sabellides.

Saccocirrus

Scoloplos

BoBr.

1863

Cllap.

1868

L.

MÜLL.

Sow. I.

JoHNST.

$1767^{\text {tb }}$ )

1865 cristata

1844 tricuspidatus

1802 ciliata.

I865 nebulosa .

Mont.

GreEFF

Risso

SAY.

L.

$1776^{4 a}$ )

1879 pelagica

1826 rudolpli.

1758 pavonina

1818 alveolata

SARS

Bobr.

$1825^{10 \mathrm{~b}}$ )

1860

BLAINV.

L.

1850 octocirrata .

MÜLL.

$\left.1815^{3 \mathrm{~b}}\right)$

$1879^{7}$ )

$1826^{3 \mathrm{~b}}$ )

$\left.1822^{4 \mathrm{a}}\right)$

$\left.1767^{4 b}\right)$

$1835^{4 a)}$

Servula .

1828 armiger

1871

L.

$1788^{3 \mathrm{~b}}$ )

1759 vermicularis .

$1766^{4 \mathrm{~b}}$ ) 
Sigalion

Sphaerodorum

Spio

spirographis

Spirorbis

Staurocephalıs

Sternaspis

Stylarioides...

Syllis

Terebellides ..

Thelepus

Tomopteris....

Travisia

Typhloscolex .

Vanadis

Vermilia......
AUD. \& M.-E. ÖRsT.

O. FABR. YN. LiI.

G'RUBE

Oтто

Chlaje SAv. in LM.

SARS

LEUCK.

Eschz.

JoHNST.

IT. Busch

Clap.

Lir.
ChutJe

$\left.1841^{3 \mathrm{c}}\right)$

1844 claparedii .

GreefF

$1 \approx 66$

1785 filicornis

1805 spalanzanii ... .

1818 borealis

1855 rubrovittatus .

1820 scutati......

$182 \mathrm{y} p$

1818 monilaris .....

1835 stroemii

1849 cincinnatus....

1825 helgolandica...

1840 forbesi

O. FABR.

Viv.

DAUD.

GRUBE

Raxz.

MÜLI.

SAV.

SARS

O. FABR.

GreefF

JoHNsT.

II. Busch

Cluap.

MöRCH

$\left.1785^{4 \mathrm{~b}}\right)$

$\left.1805^{1}\right)$

$1800^{\circ \mathrm{b}}$ )

$1855^{5}$ )

$1817^{\mathrm{bb}}$ )

$\left.1776^{4 b}\right)$

1818

$\left.1835^{4 a}\right)$

$1780^{42}$ )

$\left.1879^{7}\right)$

$\left.1843^{4 \mathrm{~b}}\right)$

$1851^{7}$ )

$\left.1870^{1}\right)$

1870 formosa

1818 multivaricosa. .

1863

Lit.: $\left.{ }^{1}\right)$ Claparède, Ann. chétop. Naples. 1868, suppl. 1870.

2) M'Intosh, Monogr. Brit. Annelids, v. 21908.

8) SAINT-JosEPH in: Ann. Sci. nat., a) 1888, b) 1894, c) 1895. d) 1898, e) 1906.

4) Mlammaren in: Öfv. Ak. Förh., a) v. 22 1865, b) v. 241867.

5) Ehlers, Borstenwürmer, 1874.

6) Eisia in: Fauna Flora Neapel, v. 16 1887.

7) GreefF in: Z. wiss. Zool., v. 321879.

3) Johnston, Cat. Worms Brit. Jus., 1865.

9) Grube in: Arch. Naturg., v. 211855.

10) Langerhans in: Z. wiss. Zool., a) v. 32 1879, b) v. 331800.

\section{Oligochaeta.}

Acanthodrilus

Aeolosoma .

Allolobophora

Alma

Aulopliorus

Bothrioneurum

Chaetogaster . .

Clitellio

Criodrilus .

Dero .

Enchytraeus

Eudrilus

Fridericia

Glossoscolex.

Henlea .

Limnodrilus

Lumbriculus

Lumbricus

Megascolex
E. Perrier 1872|ungulatus . EHrbG.

EISEN

Grube

Schmarda

STOLC

K. BAER

SaV.

Hoffmistr.

OKEN

Henle

E. Perrier

MchlsN.

F. S. LEUCK.

MCHLSN.

1831

1874

1855

1861

1886

1827

1820

1845

1815

1837

$18 \pi 1$

1889

ClaP.

1889

hemprichi.

caliginosa .....

nilotica.

furcatus

vejdovskyanum

limnaei .......

arenarius

laculum .

albidus

ellgenia

hegemon .

giganteus

ventriculosa..

GruBe

1862 hoffmeisteri . .

(L.) MÜLL.

R. T'Empl.
1844 variegatus

$(1758)$
1774 terrestris

$18+4$ caeruleus
E. Perrier 1872

EHRBG. 1831

SAV. 1826

GRUBE 1855

OKEN 1815

ŚTOLC

1886

K. BAER 1827

MÜLL.

1776

HoFFusTR. 1845

LEWY

HeNLE

KINB.

1852

VEJD.

F. TIDUCK

F. S. LeUCK. 1835

UDEK.

1854

Clap. 1862

MÜLL. 1771

(L.), MÜLL. $\left.\begin{array}{l}(1768) \\ 1774\end{array}\right\}$

R. TEMPL. 1844 


\begin{tabular}{|c|c|c|c|c|c|}
\hline Mesenchytraeus & EISEN & 1878 & i $\ldots$ & MCHLSN. & 1887 \\
\hline & RAPP & & chaetus . . & $R_{A P P}$ & 1849 \\
\hline & & & 1 & Ant. Dug. & 837 \\
\hline Moniligaster & E. Perrier & 1872 & & E. Perrier & 2 \\
\hline Nais ....... & MÜLL. & 1774 & elinguis .. & (MÜLL.) ÖRST. & \\
\hline Ocn & EISEN & 1878 & & EISEN & \\
\hline & ÖRLEY & & & Ant. Dug. & \\
\hline & CLAP. & & & Cluap. & \\
\hline & KINB. & 367 & & KINB. & \\
\hline & HOFF & & & G. L. Ha RTM. & \\
\hline $\mathrm{P}$ & E. Perrier & & & Grube & \\
\hline & SCHUARDA & & $\mathrm{co}$ & FR. MÜLL. & \\
\hline & EHRBG. & & & EHRBG. & \\
\hline & E. PER & & & F. Perrier & \\
\hline Rhynche & HofFMSTR. & & $\operatorname{limo}$ & Hoffustr. & \\
\hline . & LMI. & & lacus & L. & \\
\hline & 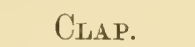 & & & & \\
\hline & BEDDARD & & & & \\
\hline 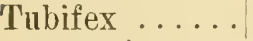 & LM. & & tubifex & MÜLL. & \\
\hline
\end{tabular}

Lit.:"Prguet in: Rev. Suisse Zool., v. 141906.

* Michaelsen in: Tierreich, v. 101900.

Michaeisen (Hamburg).

Gephyrei.

\begin{tabular}{|c|c|c|c|c|c|}
\hline osiphon . . & DIES. & 18.51 & Imülleri & DIES. & $\left.18.51^{1}\right)$ \\
\hline Bonellia .... & RoL. & 1822 & viridis . & RoL. & $\left.1822^{2}\right)$ \\
\hline chiurus & GUÉR. & 183 & echinurus . & Palil. & $\left.177 \pm^{3}\right)$ \\
\hline 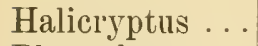 & SIEB. & $18+9$ & spinulosus & SIEB. & $\left.1849^{5}\right)$ \\
\hline na. & F. S. LEUCK. & 1828 & vulg & BLAINV. & \\
\hline & LM. & 1816 & ca & Lал. & \\
\hline & L. & 1767 & Inude & L. & \\
\hline alassema & GäRT'N. & 177 & (nes & GÄRTN. & \\
\hline
\end{tabular}

Lit.: 1) Selenka. Bülow, De Man, Sipunculidae, 1883.

2) Lagaze-Duthigrs in: Ann. Sei. nat., ser. 4, v. 101858.

3) Spengec in: Zool. Jahrb. Syst.. v. 331912.

4) Spenger in: Verh. D. zool. Ges., 1912.

5) Théel in: Svenslia Ak. Handl., v. 401906.

${ }^{6}$ H. L. Jayeson in: Zool. Jahrb. Anat., v. 121899.

Spengel (Gießen).

Hirudinei. 


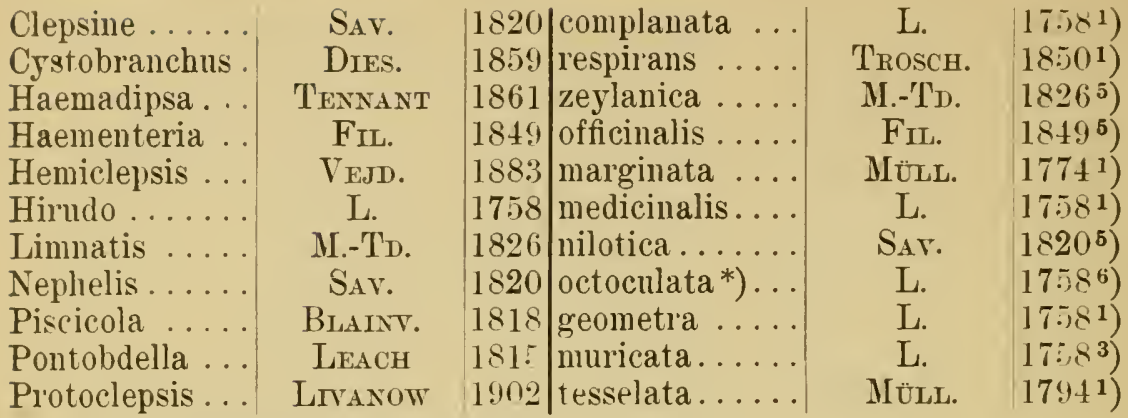

Lit.: 1) Johansson in: Brauer, Süßw., v. 131909.

2) Grube in: Mliddendorff, Reise Sibir.. v. 21851.

3) Blanchard in: Boll. Mus. Zool. Anat. comp., v. 9.

4) Johansson in: Öfvers. Vet. Ak. Förh., v. ño 1898.

5) Leguckart, Paras., ed. 21886 - 1901.

6) Johassson in: Zool. Anz.. v. 361910.

L. Johansson (Göteborg).

Rotatoria.

Aluraea..... Eн Eнвg.

Gusse

PALL.

Asplanchna

EHRBG.

Callidina

Gosse

Cathypna .....

Colurus. . .

EHRBg.

Conochilns

EHRBG.

Dinocharis ....

Euchlanis

EHRBG.

EHRBG.

Floscularia....

Hydatina

Cuv.

EHRBg.

Lacinularia

SchweIGg.

Melicerta .....

Microcodon

Schraxk

Monostyla

Oecistes

Pedalion

Philodina

Polyarthra

EHRBg.

Ehrba.

EHRBG.

Hetos.

EHRBG.

Pterodina.....

EHRBG.

EнRвG.

Rattulus..... Lis.

Rotifer

Cuv.

Salpina .

EhrbG.

Stephanoceros

EнrbG.

$1830 \mid$ aculeata .

1850 priodonta .

1766

1830 e

1886 luna

1830 uncinatus

1834 rolvox

1830 pocillum

1830 triquetra

1798 orn

1830

1820 socialis

1803 ringens

1830 clavus

1830 c

1834

1871 mira (mirum)

1830 roseola

1834 platyptera

1830 patina.

1816 l'attus .

1798 vulgaris

1830 mucronata

1832 eichhorni
EнrbG. 1832

Gusse 1850

EhrbG. 1830

EhrbG. 1830

MÜLL. 1776

NÜLL. 1773

EHRBg. 1834

MÜLL. 1776

EHRBG. 1838

Енквя. 1830

MÜLL. 1773

L. 1758

L. $1 \quad 1758$

Eнrbg. 1830

MÜLL. 1786

EHRBG. 1834

Heds. 1871

EHrbG. 1832

FHrbG. 1838

MÜLL. in 1783

Hermane

MÜLL.

Schraxk

MÜLL.

EнrbG.
1776

1801

1786

(non 1773)

1832 
Synchaeta .... EHrbG. $|1832|$ pectinata ..... Eн Eнrв. $\mid 1832$ Triarthra ..... Eнвв. 1832 longiseta ..... EнRв. 1834 \begin{tabular}{l|l|l|l|l|} 
Trochosphaera. & SEMP. & $1872 \mid$ aequatorialis .. & SEMP. & 1872
\end{tabular} Lit.: Hudson \& Gosse, Rotifera, 1886, suppl. 1889.

Gastrotricha.

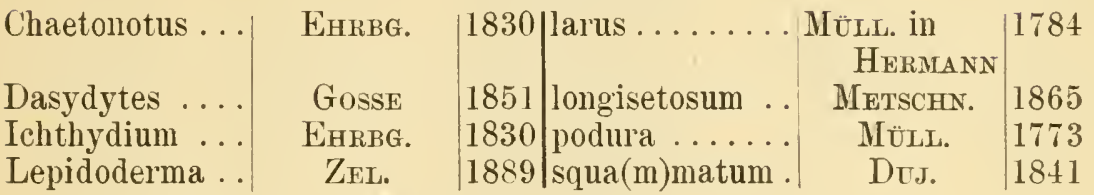

Lit.: ZeLinka in: Z. wiss. Zool,, v. 491889.

\section{Echinoderidae.}

Echinoderes

(Echinodère). DuJ. $|1851|$ dujardini

Cuap. 1863

Lit.: Schepotieff in: Z. wiss. Zool., v. 881907.

Rotatoria, Gastrotricha, Echinoderidae von Collin (Berlin).

(Anhang) Solenogastres.

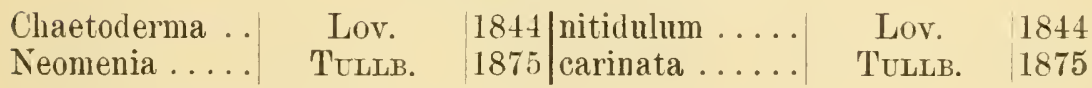

Lit.: THiele in: Tierreich, v. 381913.

\section{Crustaceı.}

Branchiopoda.

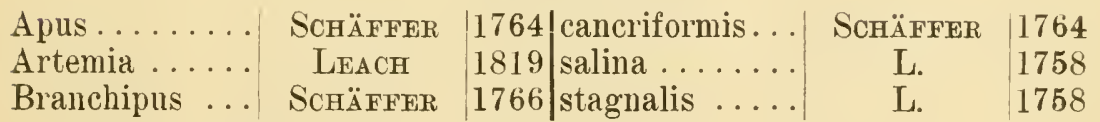

Lit.: Gerstaecker in: Brons's Kl. Ord., v. $5 \mathrm{I}$.

Cladocera.

\begin{tabular}{|c|c|c|c|c|c|}
\hline Acantholeberis & LILLJ. & 1853 & curvirostris & MÜLL. & $\left.1776^{1}\right)$ \\
\hline Acroperus ... & BAIRD & 1843 & harpae .... . & $\mathrm{B}_{\mathrm{AIRD}}$ & $\left.1835^{1}\right)$ \\
\hline lona....... & BAIRD & 1843 & quadrangularis & MÜLL. & $\left.1785^{1}\right)$ \\
\hline lonella & O. SARS & 1862 & excisa ......... & S. FISCH. & \\
\hline lonopsis. & O. Sars & 1862 & elongata..... & O. SARS & 10 \\
\hline Sono & O. SARS & 1862 & ingtus & O. SARS & \\
\hline gis & O. SARS & 1897 & cylir & O. SARS & \\
\hline se & BAIRD & 846 & longirostris & MIÜLL. & \\
\hline Os & J. Rісн. & & & J. RICH. & \\
\hline thotrephes & LEYDIG & 1860 & & LEYDIG & \\
\hline & $\mathrm{B}_{\mathrm{AIRD}}$ & & res & SснӧDL. & \\
\hline 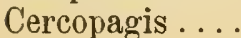 & O. SARS & & & GRIMI & \\
\hline
\end{tabular}


Ceriodaphnia .

Chydorus .....

Dadaya ......

Daphnia......

Diaphanosoma .

Dumheredia ...

Eurycercus ...

Evadne.

Graptoleberis. .

Holopedium ...

Ilyocryptus

Kurzia

Latona

Lathonura ....

Leptodora ....

Leptorhynchus.

Leydigia .

Limnosida

Macrothrix

Moina

Moinodaphnia

Monospilus .

Ophryoxus

Penilia

Peracantha

Pleuroxus.

Podon.

Polyphemus...

Pseudosida . .

Scapholeberis

Sida

Simocephalus .

Streblocerus. .
DaNA

LeACH

O. SARS

MIÜLL.

S. Fisch.

KING

BAIRD

Lov.

O. SARS

ZADD.

O. SARS

DyB.\&GROCH.

Stradus

LLLJ.

LILLJ.

HERRICK

KunZ

O. SARS

BAIRD

BAIRD

Herrick

O. SARS

O. SARS

DaNa

BAIRD

BAIRD

LILLJ.

MÜLL.

HERRICK

SсHÖDL.

Straus

SCHÖDL.

O. SARS
1853 reticulata .

1819 splaericus

1901

1785

1851 brachyurum ...

1853

$18+3$ lamellatus

1836

1862

1855

1861

nordmanni

testudinaria

gibberum .

1894 lati

1820

1853 rectirostris.

1861 kindtii

= hyalina

1874 quadrangularis

1861 frontosa

1843 rosea

1850

1887 macleayi ......

1861

1861

184.

1843

$18+3$

1853 intermedius

178:

1884

1858 mucronata....

1820 crystallina

1858 vetulus . .

1862 serricaudatus . .
1884 falcatus .....

J LR.

$\left.18 \cdot 0^{1}\right)$

MÜLL.

DADAY

GEER

IIEV.

KING

MÜLL.

Lov.

S. FIsch.

ZADD.

LIEV.

KuRZ

MÜLL.

MÜLL.

Focke

LILLJ.

O. SARS

$\left.1785^{1}\right)$

$\left.1890^{\circ}\right)$

17一⿻

$\left.18+8^{1}\right)$

$\left.1853^{\circ}\right)$

17 (A., 1$)$

$\left.1836^{1}\right)$

LEYdig

O. SARS 1861')

JUR.

JUR.

Krver

O. SARS

O. SARS

J. Rісн.

MÜLL.

MÜLL.

LILLJ.

L.

BIRGE

MÜLL.

$1820^{1}$ )

$1820^{2}$ )

$\left.18.53^{9}\right)$

$\left.1862^{1}\right)$

$\left.1861^{1}\right)$

$\left.1895^{8}\right)$

$\left.1785^{1}\right)$

$\left.178.5^{1}\right)$

$\left.18.53^{1}\right)$

$\left.1761^{1}\right)$

MÜLL.

MIÜLL.

$1910^{5}$ )

$\left.178.5^{1}\right)$

$\left.1776^{1}\right)$

(1766)

S. Fisch. 18491)

Lit.: 1) Lilujkborg in: Acta Soc. Upsal,, ser. 3 v. 19 1901. (Cladoc. suecica).

2) Leydig, Daphniden 1860.

3) J. Richard in: Ann. Sei. nat.. ser. 7 v. 181895 (Revis. Cladoc.).

4) Keilhack in: Brauer, Süßw, v. 101909.

5) Stingelin in: Mém. Soc. Neuchâtel. v. 5, 1913.

6) Stingeuin in: Zool. Jahrb. Syst., v. 201904.

?) Stingeuin in: Zool. Jahrb. Syst., v. 211904.

8) G. O. SARs in: Annuaire Mus. St. Pétersb., v. 21897.

9) Herrick \& Turner in: Rep. Surv. Minnesota. ser. 21895.

Stingelin (Olten).

Ostracoda.

Asterope

Рнш.

1840 elliptica

PhIL.

1840

Candolla

MIÜLL.

1776 
Conchoecia....

Cypridina .

Cypris

Cythere.

Gigantocypris

Halocypris . . .

Notodromas

Philomedes ... \begin{tabular}{l|l|l|} 
DAxA & 1852 & elegans . . . . . \\
M.- E. & 1840 & meditelranea . \\
MÜLL. & 1776 & pubera . . . . . \\
MÜLL. & 1785 & lutea. . . . . . .
\end{tabular}

G. W. MÜLL.

DANA

LILL.J.

LILLJ.
1895 agassizi

1852 int

1853

1853|brenda.
O. SARS
1866
O. Costa
MÜLL.
1845
MÜLL.
1776
1785

G. IV. MÜLL. 1895

DANA

1852

MÜLL.

IV. BAIRD
1776

1850

Lit.: G. W. Müller in: Tierreich, v. 311912.

\section{Copepoda.}

a) pelagica

Acartia.

Anomalocera.

Calanus

Calocalanus ...

Candacia

Canthocamptus

Centropages ...

Corycaeus

Cyclops ......

Diaptomus ....

Ectinosoma

Euchaeta .....

Harpacticus ...

Limnocalanus

Metridia......

Oithona

Oncaea

Pontella

Sapphirina .

Setella

Temora

Thalestris
DANA

R. TEMPL.

LEACH

Gresbr.

DANA

WESTW.

KRÖYER

DANA

MÜLL.

WESTW.

BOECK

Phil.

M.-E.

O. SARS

BOECK

IV. BAIRD

PHIL.

DANA 1846|longiremis ....

1837 patersoni

1816 finmarchicus

1888 pavo

1845 pectinata

1836 staphylinus

$\left\{\begin{array}{l}1848 \\ 18+9\end{array}\right\}$

1845 speciosus

1776 strenuus .

1836 castor .

$186 \pm$ atlanticum

1843

$18+3$ nol'vegica .... .

1838 chelifer.

1863 macrurus

1864 longa

1843 similis .

1843 venusta

1852 a

J. V. Thomps. 1829 nigromaculata . Dana W. BAIRD

Claus
$18 \pm 6$ gracilis .

1850 longicornis

186:3|longimana
LILLJ.

R. TEMPL.

GunN.

DANA

G. Brady

JUR.

LILLJ.

DANA

G. A. FISCH.

JUR.

(G. Buad $1820^{\circ}$

G. BRADY \& $1873^{1}$ )

ROBERTS.

BOECK

MÜLL.

O. Sars

LUBB.

Clauds

Phil.

M.-E.

Clatus

D ANA

MÜLL.

Clutus

$\left.\left.1873^{3}\right)^{5}\right)$

$\left.1785^{1}\right)$

$1863^{3}$ )

$\left.\left.1854^{3}\right)^{5}\right)$

$1866^{2}$ )

$\left.1843^{2}\right)$

$1840^{3}$ )

$\left.1863^{2}\right)^{5}$ )

$\left.18 \pm 6^{2}\right)^{5}$ )

$\left.1792^{3}\right)^{5}$ )

$1863^{3}$ )

Steder (Innsbruck).
b) parasitica.

Achtheres ....

Anchorella....

Argulıs

Caligus

Cecrops

Nordy.

Cuv.

MÜLL.

MÜLL.

LFACE

Chondracanthus
Nordm.

$\left.1832^{6}\right)$

MÜLL.

L.

MÜLL.

LEACH

KRÖYER $\left.1777^{6}\right)$

$\left.1758^{6}\right)$

$\left.1785^{6}\right)$

$1816^{6}$ )

$1837 / 8^{7}$ ) 
Ergasilus

NoRda.

1832 |nanus

BENED.

$1870^{6}$ )

Lernaea

L.

\section{7}

Lernaeocera.

Bluatvv.

Lernaeopoda . .

Blainv.

Notodelphis ...

Pandarus .....

AlLLM.

LEACH

Penella.......

OkEN

\section{2}

1822

1847 .

1816 bicol

1815 orthagorisci ...
L. $1767^{6}$ )

L. $\quad 1761^{6}$ )

L. $1761^{6}$ )

Thor. 18591)

Leach $1816^{6}$ )

P. WRIGHT $1870^{6}$ )

Lit. : 1) G. Brady, Cop. Brit. Isl., v. 21880.

2) Giesbrecht in: Fauna Flora Neapel, v. 191892.

3) Giesbrecht \& Schmeil in: Tierreich, v. 61898

4) Schmeil in: Zool., fase. 151892.

5) v. BreEMeN in: Nord. Plaukton. v. 4 pars 81908.

6) T. \& A. Sсотт, Brit. paras. Cop., 1913.

7) Clauds, Lehrb. Zool., ed. 21883.

\section{Cirripedia.}

Anelasma ..... D D D R.

Balanus

Conchoderma..

Coronula.....

Cryptophialus

Kochlorine ....

Lepas

Peltogaster ...

Pollicipes .....

Proteolepas ...

Saceulina a. . T. Y T

Costa

Olf.

LM.

DARW.

NOLL

L.

RATHKE

LEACH

DARW. 1851|squalicola . .

1778 tintinnabulum

1814 auritum

1802 diadema

1854 minutus

1875 hamata

1767 anatifera

$18+2$ paguri

1817 com

185
Lov.
L.
L.
L.

DARW.

NoLL

L.

RATHKE

LEACH

DARW. $\left.1844^{1}\right)$

$\left.1767^{2}\right)$

$\left.1767^{1}\right)$

$17672)$

$1854^{2}$ )

$1875^{3}$ )

$1767^{1}$ )

$\left.18+2^{4}\right)$

$\left.1824^{1}\right)$

$\left.1854^{2}\right)$

J. V.Thomps. $\left.1836^{4}\right)$

LFACH

GM. $\left.1824^{1}\right)$

$\left.1791^{2}\right)$
Tetraclita..... SснUм.

1817 vulgare $\ldots \ldots \ldots$

Lit.: 1) Darwin, Mlonogr. Cirrip. Lepad., 1851.

2) DARW1N, Dionogr. Cirrip. Balan., $1 \times 54$.

3) Grover, Monogr. Cirrip., 1905.

4) Gerstaecker in: Brons's Kl. Ord., $5^{1}$.

\section{Leptostraca.}

Nebalia..... Le. LeaCh $|1814|$ bipes........ O. Fabr. $\mid 1780$

Lit.: Ortmann in: Bronn's Kl. Ord., v. $\tilde{n}^{2}$.

Amphipoda.

\begin{tabular}{|c|c|c|c|c|}
\hline Amathilla. & $\mathrm{BATE}_{\mathrm{AT}}$ & 1863|homari . . & F. & (17791) \\
\hline Ampelisca & KRÖYER & $18+2$ eschrichti & KRÖYER & $\left.18+2^{1}\right)$ \\
\hline mplitoë . & LEACH & 1813 rubricata & Mont. & 1808 \\
\hline 10 & KRÖYER & 1838 nugax ... & Phipps & $177 t^{1}$ \\
\hline prella & Lx. & 1818 linearis . & L. & 1761 \\
\hline iium & Latr. & 1807 grossipes & L. & ; 1 ) \\
\hline amus. & LM. & $\left.1801\right|_{\text {ceti ..... }}$ & L. & \\
\hline
\end{tabular}


Gammalus .... F.

Hyperia

Niphargus

Orchestia

Oxycephalus

Plironima .

Platophium

Podocerus

Pontoporeia

Rhabdosoma

Talitrus
F.

LATR.

Schote.

LEACH

M.-E.

LATR.

DANA

LEACH

KRÖYER

A. White

LATR.
$1793 \mid$ pulex

182:3 galba

1849 puteanus

1814 littorea.

1830 piscator

1802 sedentaria

1852 variegatum

181: pulchellus.

1842 femorata

1850 lineatum

1802 locusta.

\begin{tabular}{c|l} 
GEER & $\left.1778^{1}\right)$ \\
MonT. & $\left.185^{1}\right)$ \\
C. L. KOCH & $\left.1835^{4}\right)$ \\
MonT. & $\left.1808^{1}\right)$ \\
M.-E. & $\left.1830^{3}\right)$ \\
ForsK. & $\left.1775^{2}\right)$ \\
LEACH & $\left.1813 / 4^{2}\right)$ \\
LEACH & $\left.1813 / 4^{2}\right)$ \\
KRÖYER & $\left.1842^{1}\right)$ \\
M.E. & $\left.1840^{3}\right)$ \\
PALL. & $\left.1772^{1}\right)$
\end{tabular}

Lit.: ${ }^{1)}$ G. O. Sars, Crust. Norway, Amph., 1890-1895.

2) Bate \& Westwood, Brit. sess. Crust., v. 1.2. 1861-1868.

3) Claus, Platysceliden 1887.

4) Keilhack in: Brauer, Süßw., v. 111909.

Reibisch (Kiel).

Isopoda.

\begin{tabular}{|c|c|c|c|c|c|}
\hline Aega........ & LEACH & & |psora ...... & Penn. & \\
\hline Anilocra... . & LEACH & 1818 & editerranea. & LEACH & \\
\hline nthura ... & LEACH & 181:3 & 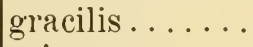 & MonT. & $\left.1808^{2}\right)$ \\
\hline & LEACH & 1814 & sp & O. SARS & $\left.1858^{1}\right)$ \\
\hline is $\ldots$. & Crv. & 1829 & & $\mathrm{SAB}_{\mathrm{AB}}$ & \\
\hline adillidium & BRANDT & 1830 & vulgare. & LAtr. & $\left.1804^{1}\right)$ \\
\hline & GEOFFR. & 1764 & $\mathrm{aq}$ & L. & $\left.758^{1}\right)$ \\
\hline & CARD. & & col'nis . . . & J. Sow. & \\
\hline & Hesse & & & RAтнке & $\left.1843^{1}\right)$ \\
\hline 0 & LATR. & 1804 & squillarum . . . & LATR. & $180+1)$ \\
\hline & LEACH & & lis .... & LILLJ. & \\
\hline & R. Kossmann & & $p y$ & RАTHKE & \\
\hline & F. & & & L. & \\
\hline & 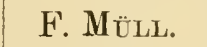 & 1862 & lanae. . & F. MÜLL. & \\
\hline & O. SARS & & $\cdots$ & O. SARS & $\left.3^{1}\right)$ \\
\hline & LEACH & & & LEACH & \\
\hline us . . & EIGHTS & & & Еіянтs & $\left.3^{1}\right)$ \\
\hline & LEACH & & & Moxт. & $\left.4^{1}\right)$ \\
\hline & CoRn.\&PAnc. & & $1 \mathrm{~S} \cdot \ldots$ & ConN. & 1 \\
\hline - & $\mathrm{F}$ & 1798 & bal & PaLL. & $\left.2^{1}\right)$ \\
\hline & LEACH & & & O.Feler. & $\left.0^{1}\right)$ \\
\hline & LEACH & 1813 & & LEACH & $\left.1813^{1}\right)$ \\
\hline & F. & 17 & & L. & $\left.1767^{x}\right)$ \\
\hline & LEACH & & & RАTHKE & $\left.1799^{1}\right)$ \\
\hline & BOECK & & elí.... . & GOODSIR & $\left.1842^{1}\right)$ \\
\hline & L. & 1767 & $\operatorname{arins} . .$. & Cov. & 1792 \\
\hline & Latr. & & & LATR. & $\left.1804^{1}\right)$ \\
\hline rerons . . . . . & LEACH & & paradoxa .... & F. & 1787 \\
\hline
\end{tabular}




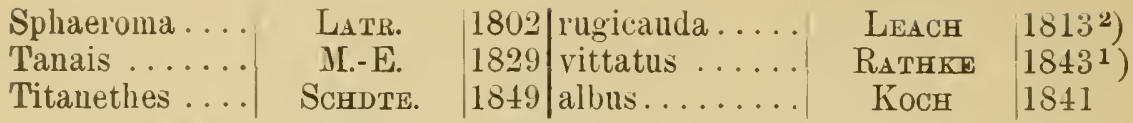

Lit.: URTMANN in: Bronn's Kl. Ord., v. 5 II.

1) G. O. Sars, Grust. Norway, v. 2 1sop.

2) Bate \& Westwood, Hist. Brit. sess. Crust., v. 2 1863/8.

Stomatopoda.

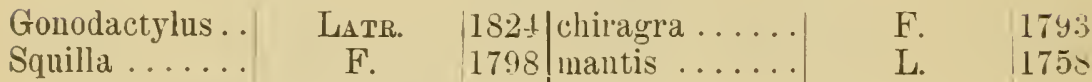

Lit.: Ortmann in: Bronn's Kl. Ord., v. $\tilde{o}^{2}$.

\section{Mysideae.}

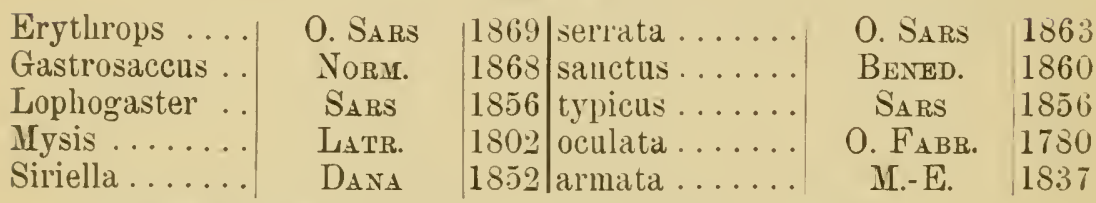

Lit.: ZimMer in: Nord. Plankton, v. 3 pars 61909.

\section{Euphausidae.}

Euphausia .... Dana $\quad 1852 \mid$ pellucida.
Decapoda.

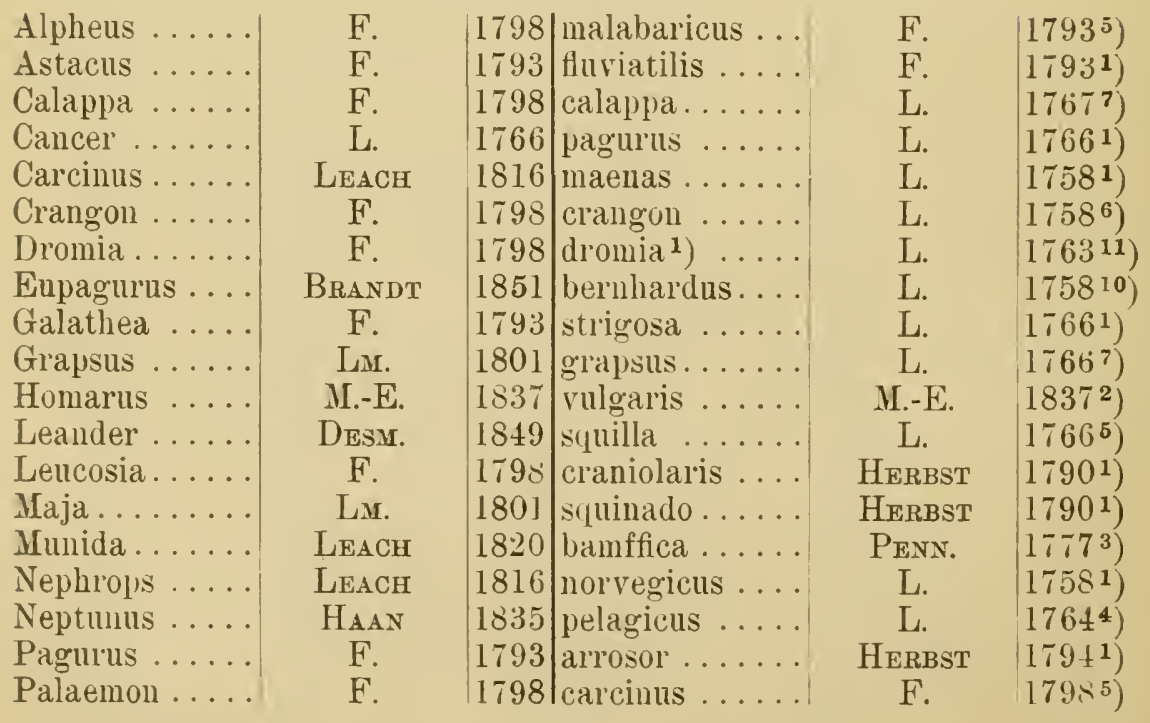

1) non dormia err. 


\begin{tabular}{l|c|c|c|c|c} 
Pandalus .... & LEACH & 1816 & annulicornis ... & LEACH & $\left.1816^{9}\right)$ \\
Penaeus .... & F. & 1798 & monodon ..... & F. & $\left.1798^{8}\right)$ \\
Portunus .... & F. & 1798 & depurator ..... & L. & $\left.1766^{7}\right)$ \\
Sesarma .... & SAY & 1817 & quadratum ... & F. & $\left.1793^{7}\right)$
\end{tabular}

Lit.: 1) HrLler, Crust. südl. Eur., 1863.

2) Milne-Edwards, Hist. nat. Crust., v. $1-3$ 1834-40.

3) Minne-Edwards \& Bouvirr in: Exp. Travailleur \& Talisman 1900.

4) Mrne-Edwakds, Étud. Crust. Portuniens, 1861.

5) Ortmann in: Zool. Jahrb. Syst , v. 5-7 1890-93.

6) Ortmann in: P. Ac. Philad, 1895.

7) Alcocr in: J. Asiat. Soc. Bengal, v. 64-69 1895-1900.

8) Atcock, Cat. Indian Dec., 1906.

9) Calman in: Ann. nat. Hist.. ser. 7 v. 31899.

10) Benedict in: P. U. S. Mus., v. 231901.

11) Ihle in: Siboga Exp., 1913.

BALSS (IIünchen).

Xiphosura.

Limulus ..... MÜLL. $|1785|$ polyphemus ... $\quad$ L. $\mid 1758$

Lit.: Geretaecker in: Bronn's Kl, Ord., $v 5$ I.

\section{Arachnoider.}

Pantopoda.

\begin{tabular}{|c|c|c|c|c|}
\hline Ammothea & LEACH & 1814 echinata & HODGE & 186 \\
\hline Collossendeis & $\mathrm{J}_{\mathrm{ARZ}}$. & 1870 proboscidea & $\mathrm{S}_{\mathrm{AB}}$ & 182 \\
\hline Nymphon ... & F. & 1794 grossipes . & O. $F_{A B R}$. & 178 \\
\hline Pallene....... & ЈоHNST. & 1837 brevirostris & JoHNST. & 18 \\
\hline Phoxichilidium & M.-E. & 1840 femoratum. & RATHKE & 74 \\
\hline Pycnogonum . & BRÜNN. & 1764 litorale ... & STRÖM & 6 \\
\hline
\end{tabular}

Lit.: G. O. SARS in: Norske Nordhavs Exp., v. 61891.

HeLFER (Rerlin).

\section{Myzostomidae.}

Myzostoma ... F. F. S. Lwuck. 1827|cirriferum ....|F. S. Leuck. $\mid 1827$

Lit.: Grapf, Genus Myzostoma, 1877.

\section{Linguatulidae.}

Pentastoma ... RUD. $|1809|$ taenioides..... $\mid$ RoD. $\mid 1809$ Lit.: R. Lgukart, Pentastomiden, 1860.

\section{Tardigrada.}

Echiniscus .... S. Schultze $|1840|$ bellermanni ... S. SCHULtze $\mid 1840$ Macrobiotus... S. SchULtze 1834 hufelandii..... S. SCHULtZe 1834 Milnesinm .... $\mid$ Doy. $|1840|$ tardigradum... $\mid$ Doy. 1840 Lit.: Plate in: Zool. Jahrb. Anat., v. 31888. 


\section{Acarina.}

Amblyomma... Analges

Al'gas

Arrhenurus

Atax . .

Bdella .

Cheyletus

Chorioptes

Damaeus

Demodex

Dermacentor

Dermanyssus .

Dermatocoptes

Dimorphus ... .

Diplodontus

Erythraeus

Evlais.

Gammasus

Glycyphagus

Haemophysalis

Halacarus

Hermannia

Hyalomma

Hydi'achna .

Hygrobates

Ixodes

Limnesia

Limnochares

Listrophorus

Myocoptes

Notaspis

Oribata.

Phytoptus

Psoroptes

Pteroptus

Rhipicephalus

Rhizoglyphus .

Sarcoptes

Tetranyclius.

Trombidium .

'Tyıoglyphus
C. L. КосH

Nitzasch

LATR.

Ant. Dug.

F.

LATR.

LATR.

Gerv.

C. L. KoCH Ow.

C. L. KосH Ant. Dug.

FÜRSTB.

HALLER

Axt. Deg.

Latr.

LATR.

LATR.

Herixt

C. L. KOCH

Gosse

NIC:

C. L. KOCH

MUÜLL.

C. L. $\mathrm{KoCH}$

LATR.

C. L. KocH

LATR.

PGst.

Claap.

Herir.

LATR.

DuJ.

Gerv.

DUF.

C. L. Koch

Clap.

LATR.

DUF.

F.

LATR.
1844 cajennense

1818 passerinus

1796 reflexus

1834 globator

180 ) bonzi

1797 longicornis

1796 eruditus .

1859 equi

1835

$18+3$ folliculorum

1844 reticulatus

1834 avium .

1861 equi

1877 columbae

$183+$ decipiens

1806 parietinus

1796 extendens

1802 coleoptratorum .

1838 domesticus . . . .

1844 concinna.

185.5 ctenopus

1855 picea. .

1844 aegyptium

1776 cruenta.

18.37 longipalpis .

179 ) ricinus

1835 miaculata.

1796 holosericeus

1861 pagenstecheri.

1869 musculinus . . . .

180.1 bipilis

1802 alata.

1851 vitis

1841

1832 vespertilionis

1844

1869 sanghinens ....

1869 robini

1806 scabiei

$18: 32$ telarius

179: holosericeum

1796

F.
L.
F.
MÜLL.
CLAP.
L.
SCHRANK
GERL.
C. L. KoCH
G. SIM.
F.

ANT. Dug.

Hering

Bт्CHH.

MÜLL.

Hersi.

MÜLL.

L.

GEer

C. L. $\mathrm{KoCH}$

Gusse

C. L. KOCH

$\mathrm{L}$.

MÜLL.

HERM.

L.

IIÜLL.

LATR.

Heller

C. L. KocH

Ненм.

Hery.

Laxiols

GERL.

Herur.

LATR.

Clap.

GEeR

L.

L.

LATR. (7n-4)

$\left.17.58^{2}\right)$

$\left.1794^{4}\right)$

$1776^{3}$ )

$\left.1869^{3}\right)$

$\left.17.58^{5}\right)$

$\left.1781^{6}\right)$

$1857^{2}$ )

$\left.1835^{-1}\right)$

$18+22)$

$\left.179 t^{4}\right)$

$\left.1834^{7}\right)$

$\left.1838^{2}\right)$

$\left.1869^{2}\right)$

$178 I^{3}$ )

$1804^{6}$ )

$\left.17.6^{3}\right)$

17.586 )

$\left.1778^{2}\right)$

$\left.18+t^{4}\right)$

18.5. ${ }^{3}$ )

$1840^{1}$ )

$17584)$

$1781^{3}$ )

$\left.1804^{3}\right)$

$\left.175 i^{4}\right)$

$\left.1776^{3}\right)$

$1 \leqslant\left(16^{3}\right)$

$\left.1850^{2}\right)$

$\left.1814^{2}\right)$

$18\left(14^{1}\right)$

$180+1)$

$186+8$ )

$\left.1857^{2}\right)$

$1<04^{7}$ )

$\left.1806^{4}\right)$

$1869^{2}$ )

$\left.177 \aleph^{2}\right)$

$\left.1758^{6}\right)$

$\left.1758^{6}\right)$

$\left.179 i^{2}\right)$

Lit.: 1) Мichael in: 'Tjerreich, v. 31898.

2) Canestrini \& Kramer in: Tierrejch. $v .71899$.

3) Piersia \& Lohmann in: Tierreich, v. 131901.

4) Neumann in: Tierreich, v. 261911. 
Lit.: 5) Thor in: Zool. Anz., v. 281905.

6) C. L. KocH, Übers. Arach., fase. 31842.

7) IĹgrin in: J. Anat. et Physiol., v. 121876.

8) Landois in: Z. wiss. Zool., v. 141864.

Araneida.

Agelena

Amaurobius ...

Anyphaena....

Araneus b) ....

Argiope ${ }^{1}$ ).....

Argyroneta ...

Asagena ......

Atypus

Avicularia ....

Chiracanthi-

$u \mathrm{~m}^{2}$ ) ......

Clubiona

Coelotes

Cteniza

Ctenus

Dendryphantes

Dictyna

Dinopis ${ }^{3}$ ) ....

Dolomedes

Drassodes

c)

Dysdera ......

Eresus.

Erigone.......

Ero

Filistata .....

Gasteracantha.

Gnaphosa .....

Hahnia

Harpactes

Heliophanus...

Hersilia......

Heteropoda ...

Hyptiotes ${ }^{5}$ )...

Latrodectus ...

Linyphia......

Lycosa

Marpissa

Meta
WALCK.

C. L. KOCH

Sund.

(Clemek

$\mathrm{SAv}$.

LATR.

SUND.

LATR.

LiI.

C. L. Косн

LATR.

BLACKW.

LATR.

W ALCK.

C. L. KосH

Sund.

A. M'Leay

LATR.

WEsTR.

LATR.

ITALCK.

SAV.

C. L. K осH

Latr.

Guer.

LATR.

C. L. KocH

TEMPL.

C. L. KocH

SAV.

LATR.

W ALCK.

IVALCK.

LATR.

LATR.

C. L. KocH

C. L. KoCH
180 . labyrinthica...

$18: 37$ claustrarius ...

1833 accentuata ....

1757) algulatus .... .

1826 sericea. .

1804 aquatica

1833

1804

1818

phalerata ....

subterraneus . .

avicularia .....

1839 nutrix

1804

1841 saxatilis

1829 sauvagesii . .

1805

1837

$18: 3$ ar undinacea ...

1839

1804

1851 lapidosus

1804

1805

1826

1837

1837

1810

1830

1804

1841

1834

1833

1826

1804

1837

$180:$

punctoria

cinnabarinus

vagans........

tuberculata

testacea

cancriformis...

melanogaster . .

pusilla.......

hombergi

cupreus.......

candata

regia

paradoxus....

tredecim-

guttatus

1804 triangularis

1804 amentata

1846 muscosa

1836 menardi
(CIERCK

HAHN

WALCK.

(Clikrck

Sav.

(Clerck

Panz.

Rossi

L.

C. L. KoCH

1839 h) 14 )

(Clerek

1757) ${ }^{2}$ )

Blackw.

RossI

$1834^{6 \mathrm{a}}$ )

WALCK.

(Clerek

L.

$\left.1790^{1}\right)$

180.511)

$1857)^{2}$ )

17581)

A. M'Leay

$1839^{5}$ )

(Clerek

W ALCK.

LAтк. ${ }^{4}$ )

OL.

SAv.

1757)a ${ }^{6 \mathrm{c}}$ )

GeER

LATR.

L.

LATR.

C. L. КосH

SCOP.

WALCK.

Sav.

F.

C. L. КосH

$180.21)$

$\left.1804^{1}\right)$

$\left.1789^{1}\right)$

$18 \cdot 64)$

$\left.1778^{2}\right)$

1810 1 )

$1758^{9}$ )

$\left.1804^{1}\right)$

$\left.18+1^{2}\right)$

176.31)

$1802^{2}$ )

$\left.1826^{4}\right)$

$1793^{13}$ )

$1834^{1}$ )

RossI

17901)

(Clekck

$\left.1757 d)^{7}\right)$

(Clerch

1757)i) ${ }^{12}$ )

(Clerek

$1757)^{1}$ )

LATR.

$1804^{2}$ )

Anm.: 1) Argyope, 2) Cheiracanthium, 3) Deinopis, 4) Latr. non Villers, 5) Uptintes. 


\begin{tabular}{|c|c|c|c|c|c|}
\hline & W'ESTR. & & & $W_{A L C}$ & $\left.1802^{2}\right)$ \\
\hline ena ... & SUND. & & clypeata & WALCK. & $\left.1805^{10}\right)$ \\
\hline $\left.\operatorname{nata}^{6}\right)$. & Latr. & 1804 & virescens. & (CLERCK & (1757)a $\left.{ }^{2}\right)$ \\
\hline & LATR. & $180 \div$ & vatia...... & (C'LERCK & $\left.1757^{2}\right)$ \\
\hline & A. M'Leay & 1839 & melanocephala . & A. M'Leay & $\left.1839^{5}\right)$ \\
\hline$\ldots \ldots$ & SAY. & 1826 & cell & SAv. & $\left.1826^{4}\right)$ \\
\hline & LEACH & & min & $\mathrm{F}$. & $\left.1793^{8}\right)$ \\
\hline & & & & SAv. & $\left.1826^{\text {6d }}\right)$ \\
\hline & LUC. & & & Luc. & $\left.1845^{1}\right)$ \\
\hline & WALCE. & & & Def. & $\left.1820^{1}\right)$ \\
\hline & & & $\begin{array}{r}\text { heterophthal- } \\
\text { mus ....... }\end{array}$ & $\mathrm{LA}_{\mathrm{A}} \mathrm{T}$ & $\left.1802^{2}\right)$ \\
\hline & SUND. & 1823 & & & $\left.1823^{2}\right)$ \\
\hline & Dut & & & Do & 1820 \\
\hline & $\mathrm{IT}_{\mathrm{AI}}$ & & & $\left(\mathrm{C}_{\mathrm{LI}}\right.$ & 1757)e ${ }^{2}$ ) \\
\hline & $W_{A}$ & & & Fur & $\left.1775^{1}\right)$ \\
\hline & L. KoCH & 1872 & sit & C. L. Косн & $\left.1833^{1}\right)$ \\
\hline & LATR. & 1804 & & (CLeI & (757)a) $\left.{ }^{2}\right)$ \\
\hline & LAT & 1804 & $\mathrm{t}$ & & $\left.1804^{1}\right)$ \\
\hline & & 04 & & & \\
\hline & $\mathrm{LA}_{\mathrm{A}}$ & 19 & & $\mathrm{LAT}^{\mathrm{T}}$ & 18194) \\
\hline & E. S & & $\mathrm{t}$ & (Clukrok & 1757 \\
\hline & & & & & $\left.1758^{1}\right)$ \\
\hline & E. SIM. & 64 & $\mathrm{glc}$ & F. & 1775 \\
\hline & & & & (Clemerk & $\left.6^{6 c}\right)$ \\
\hline & & & & Scop. & $\left.1763^{2}\right)$ \\
\hline & $\mathrm{LA}$ ? & & & L. & $\left.1758^{2}\right)$ \\
\hline & $\mathrm{Su}$ & & de & OL. & $\left.1789^{2}\right)$ \\
\hline & C. L. Косн & 1837 & formici & (CleERCK & \\
\hline & W ALCK. & & & (Clemere & 1757 \\
\hline & $W_{A I}$ & & & Gм. & $\left.1778^{2}\right)$ \\
\hline & & & & Latr. & $18061)$ \\
\hline & Duf. & 1820 & & LAT & 1809 \\
\hline & C. L. КосH & 1835 & cristatus & (Cleterch & $(1757) g)^{2}$ \\
\hline Zodarion ${ }^{8}$ ) & W ALCK. & & longipes & SAv. & 1826 \\
\hline or: & C. L. Косн & 1848 & spin & Sund. & $\left.1833^{2}\right)$ \\
\hline
\end{tabular}

Lit.: 1) E. Srmon, Arach. France, v. $1-7 \quad 1874-1879$.

2) Chyzer \& KUlCzÝ́SKI, Aran. Hungar., v. 1, 2 1892-1897.

3) F. CAMBRIDGE in: P. zool. Soc. London, 1896.

Anm.: ${ }^{6}$ ) Micromata. ${ }^{7}$ ) Synema, ${ }^{8}$ ) falls 1847 richtig ist, müßte "Zodarium" der "Enyo Sav." Platz machen.

Anm. von Apstein: a) Vor-Linne'ische Namen sind nach den intern. Nomenklaturregeln ungültig. Die mit einem a) versehenen Namen sind von Lisnte übernommen, müssen daher L. 1758 als Autor und Jahr tragen, b) Aramea L. 1758, c) Épeira WALCK. 1805 , diadema (ta) L. 1758, d) WALCK. 1802, e) WALCK. 1802, f) WALCK. 1802, g) WALCK. 1805, h) non WALCK., i) sub: L. saccata L. 
Lit.: $\left.{ }^{4}\right)$ Savigny (resp. Audouin in Sav.) in: Desc. Egypte, v. 1 pars 41826.

5) Mac LeaY in: Ann. nat. Hist., v. z 1839.

$\left.{ }^{6}\right)$ KulczyŃski in: Bull. Ac. Cracovie, a) 1906, b) 1907 , c) 1909 , d) 1911.

7) KULCZYŃsKI, Faune Walonyki.

8) DAHL in: It. Mus. Berlin, v. 61912.

9) $\mathrm{Ic}$ Cook, Amer. Spiders, v. 3, p 211.

10) C. L. K $\mathrm{KocH}$, Arachn., v. 1-16 1831-1848.

11) WALCKENAER, Hist. Apt., v. $1-4$ 1837-1847.

12) DAHL in: N. Acta Ac. Leop.. $v 881908$.

13) Thorelt in: Ann. Mus. Genova v. 101877.

14) C. L. Косн, Arach. Drassiden, 1866-1868.

KULCZYŃSKI (Krakau).

\section{Phalangida.}

Dicranopalpus .

Gagrella.

Gonyleptes....

Liobumum

Melanopa.....

Opilio.

Phalangium ...
DOL.

STOL.

KIRBY

E. Sім.

THOR.

Herbst

L. 1852|gasteinensis ... 1869

1819

1879 rot

1889

1798

1761

teae

horridus

rotundum

atrata.

barietinus....

colnutum

Lit.: 1) Roewer in: Abh. Ver. Hamburg, 1910.

2) Roewer in: Abl. V'er. Hamburg, 1912.

\begin{tabular}{l|l} 
Dol. & $\left.1852^{2}\right)$ \\
ThOR. & $\left.1889^{1}\right)$ \\
KIRBY & $\left.1819^{2}\right)$ \\
LATR. & $\left.1798^{1}\right)$ \\
StoL. & $\left.1869^{1}\right)$ \\
GEER & $\left.1778^{2}\right)$ \\
L. & $\left.1761^{2}\right)$
\end{tabular}

Roewer (Bremen).

\section{Pedipalpi et Scorpiones. $\left.{ }^{*}\right)$}

Telyphonus ... Latr. $\mid$ Le02|caudatus..... .

L.

1758

Lit.: Kraepelix in: Tierreich, v. 81899.

\section{Solifugae.}

\begin{tabular}{l|l|l|l|l|l} 
Galeodes .... & OL. & 1791 & alaneoides ... & PaLL. & 1772 \\
Solpuga ..... & A. Lcht. & 1796 & chelicolnis ... & A. LCHT. & 1769
\end{tabular} Lit.: Kraepelin in: 'Tierreich, v.12 1901.

\section{Pseudoscorpiones.}

Chelifel...... L. GEoffr. 1764 |cancroides.

Chernes

Chthonins....

Obisium
IENGE

C. L. LOOCH

ILL. \begin{tabular}{l|l|}
1764 & cancroides . . . \\
1855 & scorpioides . . . \\
1843 & trombidioides . . \\
1798 & muscortum . . . .
\end{tabular} \begin{tabular}{c|c} 
L. & 1767 \\
HERMI. & 1804 \\
LATR. & 1806
\end{tabular}

C. L. Косн 1843

Lit.: L. Kocn, Chernetiden, 1873.

*) Folgende 3 Genera gehören auch in die Liste, sind aber so gründlich umgetauft, $\mathrm{daB}$ es fraglich ist, ob sie ihre gebräuchlichen Namen behalten können; ich lasse sie daher als Anm. folgen:

Phrynus ..... Latr.

Scorpio

Buthus
L.

LEACH
1802 reniformis

1758 europaeus.... .

1815 afer
LATR.

L.

L.
1802

1758

1758

Lit.: Cuvier, Kègne an. ed.4 Arachn. t. 16. 17. 19. 


\section{Onychophore.}

Peripatus ..... Glud. $1826 \mid$ capensis ...... Grube 1866 Lit.: A. Sredgwick in: Quart. J. micr. Sci., v. 281888 p. $431 \mathrm{ff}$.

\section{Hyrioporte.}

\begin{tabular}{|c|c|c|c|c|c|}
\hline Alipes ....... & ІмноFе & 1854 & multicostis . . . & ТмноF & 1854 \\
\hline Blaniulus ... & Gerv. & 1836 & guttulatus .... & Bosc & 1792 \\
\hline Cryptops ... & LEACH & 1814 & hortensis .... & LEACH & 1814 \\
\hline Geophilus.... & LEACH & 1814 & fer'lugineus.... & C. KосH & 1835 \\
\hline Glomeris .... & LATR. & 1802 & marginata ... & Villers & 1789 \\
\hline Lithobius . . . . & LEACH & 1814 & forficatus... & L. & (5) \\
\hline Pauropus ..... & LuвB. & 1866 & huxleyi .... & Lетвв. & 866 \\
\hline Polydesmus ... & LATR. & 1802 & complanatus... & L. & 1761 \\
\hline Polyxenus .... & LATR. & 802 & laguruts . . . . . . & L. & 7 .) \\
\hline Polyzonium ... & BrantT & 1834 & germanicum . . & BRANDT & 831 \\
\hline Scolopendla . . & L. & 1758 & morsitans . . & L. & 1758 \\
\hline Scolopendrella. & Geriv. & 1839 & immaculata. & NEWP. & 81 \\
\hline Scutigera ..... & LaI. & 1801 & coleoptrata... & L. & \\
\hline Sphaerotherium & BRANDT & 1833 & elongatum ... & BRANOT & \\
\hline Spirobolus .... & BRANDT & $18: 33$ & maximus... & BRANDT & 1833 \\
\hline Spirostreptus . . & Braxut & $183: 3$ & jaranicus ... & BRANDT & 182. \\
\hline
\end{tabular}

Lit.: Latzel, Myriap., v. 1, 2, 1880, 1881.

1) Kraeplin in: Mt. Mus. Hamburg, v. 21) 1903.

\section{Hexriportr.}

\section{Thysanura.}

Camporlea....

II ESTW.

1842 staphyliums

L. Af.

Desoria

Ronv.

$18+1$

Bouri.

Tsotoma

HALID.

1861

lacialis

Japyx . 18.39

\section{I}

Machilis

L.

LATR.

Podural...... L L.

Smintlıums ... Litr.

1863 solifugus . . . . .

WEsTw.

Nic.

I.

Botru.

HILID.

L.

L.

\begin{tabular}{l|l|}
1758 & saccllalina ... \\
1804 & polyporla . . . .
\end{tabular}

1758 anduatica ......

I.

L.

$\left.\left(18+2^{1}\right)^{2}\right)$

$\left.18+2^{1}\right)$

$\left.1758^{2}\right)$

$\left.1839^{1}\right)^{2}$ )

$\left.1863^{1}\right)$

$\left.17581^{2}\right)$

$\left.\left.176 i^{1}\right)^{2}\right)$

1802 viridis . . . . . .

$\left.\left.1758^{1}\right)^{2}\right)$

$\left.\left.1758^{1}\right)^{2}\right)$

Lit.: 1) LubBock, Monogr. Colemb Thys.. 18:33.

2) Wahlgren in: Ent. 'Tidskr., v. 271906 p. $219 \div 270$.

\section{Oithoptera.}


Caloptenns ....

Chelidura .....

Chrysochraon .

Conoceplialus .

Decticus

Ectobia

Enpusa

Eplippigera .

Forficula

Gampsocleis .

Gomphocer'us

Gryllacris .

isyllotalpa

Gryllus .

Labia.

Labidura

Leptophyes ...

Locusta

Mantis

Meconema

Mecostethus ...

Myımecopliila .

Oecanthus ....

Oedipoda

Orplania .....

Pachytylus....

Periplaneta ...

Phaneroptera. .

Phasma

Phyllium

Pliyllodromia.

Platycleis .....

Podisma

Pseudophyllus

Psophus

Saga

Sphingonotus . .

Stenobothrus .

Stethophyma .

'Tettix.

Thamnotrizon

Tridactylus ...

Troglophilus

Tryxalis

'Tylopsis

Xiphidium
Burm.

SFrv.

I.

THUxB.

SERV.

W Es'TW.

ILL.

LATR.

L.

Fiets.

'Thunb.

Serv.

LATR.

L.

LEACH

LEACH

Finв.

GEER

L.

Serv.

Fieb.

LATR.

SERv.

LATh.

L. H. FIsch.

Finb.

Birit.

SERY.

Lснт.

ILL.

Serv.

Finb.

LATR.

SERY.

Fiet.

T. Chake.

FiEB.

L. H. FIsch.

L. H. FIsch.

T. ChiRP.

L. H. Fisch.

LATR.

KraUss

F.

Fieb.

SERY. $1839\left|\begin{array}{l}18 \text { italicus } \\ 1831\end{array}\right|$

1853

1815

1831

1839

1798

1825

1758

1853

1815

1831

1807 vulgalis

1758 campestris

1815 minor

1815

1853

1773

1758

1831

1853

1825

1831 pellucens

1825 coerulescens

1853

1853

1838

1831

1796 fasciatum

1798 siccifolium

1839 germanica

1853

1825

risea

1831

1853 stridulus .

1825 serrata.

1853 coerulans

1853 lineatus

185:3

$18+1$

1853

180 !

fuscum .

subulatus.

cinereus

1878 cavicola

1775 nasuta

1853 liliifolia

1831 fuscum
L.

T. Charp.

GERM.

T. Charp.

L.

Brulle

SERV.

I.

Herbst

Thuxb.

SERr.

LATR.

L.

L.

PAlil.

Bosc:

L.

L.

GEER

L.

Paxz.

Scop.

I.

T. Charp.

L.

I.

F.

GRAY

L.

L.

F.

Kolt.

Piet.\&SALSs. $1892^{2}$ )

L.

F.

L.

Paxz.

Pall.

I.

I.

IATR.

KOLL.

I.

F.

F.
1766

$\left.1825^{1}\right)$

1817

1825

1761

1758

1836

1831

$1758^{1}$ )

1786

1815

$\left.1831^{2}\right)$

1807

1764

$\left.1758^{1}\right)$

$\left.1773^{1}\right)$

1742

1758

1758

$\left.1771^{2}\right)$

1766

1820

1763

1764

1825

1758

1758

1793

1835

$\left.1758^{2}\right)$

$\left.1767^{2}\right)$

1793

1833

1761

1793

1766

1796

1773

1761

1789

1804

1833

1764

1793

1793 
Lit.: Redtenbacuer, Dermapt. Orthopt., 1900.

1) Bormans \& Krauss in: Tierreich, v. 111900.

2) IV. F. KirbY, Cat. Orthopt., v. I-3 1904-1910.

Rasme (Berliu).

Physopoda.

Heliothrips ... H HALD. $1836 \mid$ haemorrhoidalis Linotluips .... HALID. 1836 cerealium ..... Plysopus .... Ge Ger 1773 vulgatissimus.. Thrips ...... L. 1758 communis . . . .

Botche 1833 HALID. HALID. 1836

Lit.: UzeL, Monogr. 'Thysanoptera, 1895.

UZEL 1836 1895

\section{Psocidae.}

\begin{tabular}{|c|c|c|c|c|c|}
\hline Amphigorontia & Kolbe & 1880 & bifisciata. & Latr. & 1799 \\
\hline Atropos ..... & LEACH & 1815 & pulsatoria. & L. & 1758 \\
\hline Caecilins.... . & J. Curt. & 1837 & fuscopterus & LATR. & 1799 \\
\hline Graphopsocus & KoLBE & 1880 & cruciatus . . & L. & 1768 \\
\hline Philotarsus . & KоLBE & 1880 & flaviceps . . & S'TFPH. & 1836 \\
\hline Psocul .... & LATR. & 1794 & saltatıix. & L. & $(1746)$ \\
\hline Pterodela . & KoLBE & 1880 & pedicularia... & L. & 1758 \\
\hline tenopsocus ... & HAGEN & 1866 & immaculatus . . . & STEPH. & 1836 \\
\hline Troctes....... & Burs. & 1839 & divinatorius . . . & MÜLL. & 1776 \\
\hline
\end{tabular}

Lit.: KoLBe-Rostock, Neur. germ., 1888.

\section{Termitidae.}

\begin{tabular}{c|c|c|c|c|c} 
Calotermes.... & Hagex & 1853 & flaricollis .... & F. & 1793 \\
Termes..... & L. & 1758 & fatale ...... & L. & 1758
\end{tabular}

Lit.: Hobmgnin in: Srenslia Ak. Handl., v. 16,18 1911, 1912.

\section{Perlidae.}

Capnia

Chloroperla ...

Dictyopteryx . .

Isopteryx .....

Lenctia

Nemin

Perla

Taeniopteryx . .
A. Pictét

Newall.

A. Pictet

A. P'icteis

STLPH.

LATR.

GEOFFL.

A. PICTIT'T
$18 \pm 1$ lnigra.

1839 grammatica . .

$18+1$ microcephala . .

$18+1$ trijunctata...

1836 nigla .......

1796 valiegata .....

1764 maxima .....

1841 trifasciata ...

Lit.: Kílbe-Rostock, Neur. germ., 1888.

\section{Ephemeridae.}

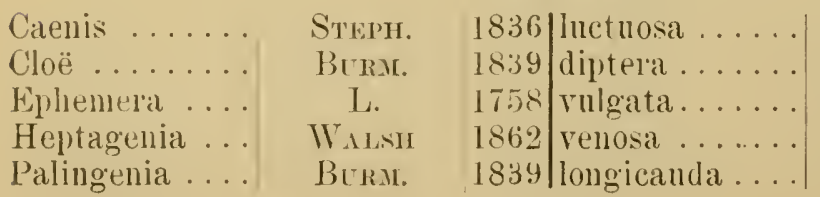

A. Picteт 1841 Scop. 1763

A. Picte:T $18 \pm 1$ SCOP. 1763 OL. $\quad$ I811 OL. 1811 Scope A. Picteit $18+1$ 1763

Lit: Kolbe-Rostock, Neur. germ., 1888. 
Embiidae.

Embia ...... Latr. |1825|savignyi ..... W W Lit.: Krauss in: Zool., Fase. 601911.

\section{Odonata.}

\begin{tabular}{|c|c|c|c|c|}
\hline Aeschua ...... & $\mathrm{F}$. & 1775 & grandis ....... & L. \\
\hline Aglion ....... & F. & 1775 & puella ........ & I. \\
\hline $\operatorname{Anax} \ldots \ldots$ & LEACH & 1815 & imperator..... & LFACH \\
\hline Calopteryx ... & LEACH & 1815 & rilgo $\ldots \ldots \ldots$ & L. \\
\hline Cordulegaster & LEACH & 1815 & annulatus . . & LATR. \\
\hline Cordulia ...... & LEACH & 1815 & aenea ....... & L. \\
\hline Epitheca ..... & Векג. & 1839 & bimaculata .... & Charp. \\
\hline Gomphus ... & LEACH & 1815 & vulgatissimus. . & L. \\
\hline Lestes .... & LEACH & 1815 & sponsa ....... & HANSEM. \\
\hline Libellula ..... & L. & 1758 & depressa...... & L. \\
\hline Lindenia . . . . & HAAN & 1826 & folcipata ..... & L. \\
\hline Macromia . . . . . & RАयв. & 1842 & cingulata ..... & Rамв. \\
\hline rthetrum .... & Newa. & 1833 & caterulescens... & F. \\
\hline Sympetrum . . & Newa. & 1833 & rulgatum . . . . . & L. \\
\hline
\end{tabular}

Lit.: Brader \& Löw, Neur. austr., $185 ̄ 7$.

Ris in: Coll. Selys-Longchamps, Catal. syst. descript, Libellulinae, 1909-1914. Grérberg (Berlin).

Neuroptera (Lit. 1).

Ascalaphus....

Bittacus

Boreus

Clrysopa

Coniopteryx ...

Hemerobius . . .

Mantispa ....

Myrmeleon ....

Nemoptera... .

Panorpa

Raphidia

Sialis

Sisyra

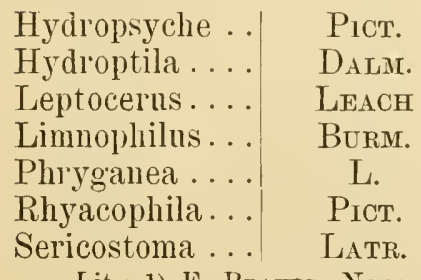

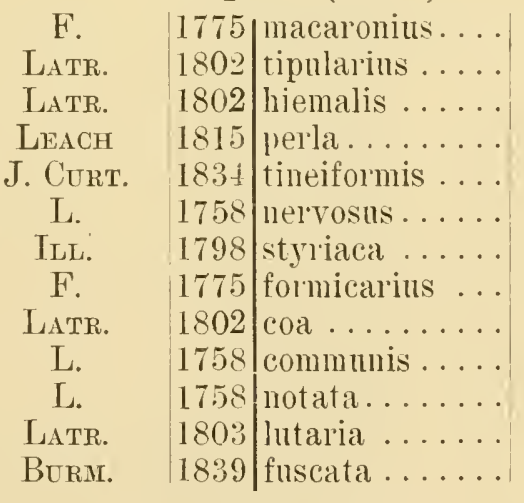

Trichoptera (Lit. 2).

Lit.: 1) F. Brader, Neur. austr., 1857.

2) Ulmer in: A. Brauer, Süßw, v. 5, 61909.

\begin{tabular}{c|c} 
Scop. & 1763 \\
L. & 1788 \\
L. & 1767 \\
L. & 1758 \\
J. CURT. & 1834 \\
F. & 1793 \\
PODA & 1761 \\
L. & 1788 \\
L. & 1758 \\
L. & 1758 \\
F. & 1781 \\
L. & 1758 \\
F. & 1793
\end{tabular}

Pict.

1834

J. Curt.

1834

1759

1758

L.

L.

1758

Pict.

Spence
1834

1826 


\section{Strepsiptera.}

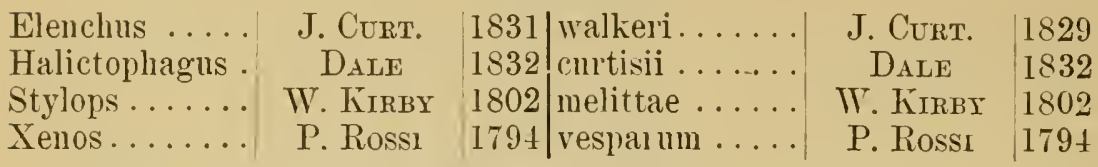

Lit.: Pierce in: Bull. U. S. Hus., v. 6h 1909.

\section{Rhynchota.}

Acalypta ..... Westw. 18t0|carinata. Acanthaspis ... A A. \& SERr. 1843 flavovaria..... Acanthocoris . . Ax. \& SERv. I843 scabrator ..... Acanthosoma . . Acetropis ..... Acocephalus... Adelphocoris . .

J. Curt. 1821 haemor'lioidale Aelia

FIEB.

Gery.

Reuter

F.

Aepophilus :... Agallia . Agapophyta... Agonoscelis ...

Aleyrodes.....

Alloeonemra ... Alydus ....... Amblytylus Anasa ........ Ancyrosoma... Aneurus ...... . Anisops Anisoscelis .... Anoplocnemis. .

Antestia

\section{Sign.} J. Cirtit.

LAP. Spix.

LATR.

F. Löw. F.

Fieb. 1858 1833 carinata ..... 1896 nelvosus

.

Paxz. $1806^{6}$ ) НА̀H $1834^{5}$ ) F.

L.

H.-ScH. SCHRANK

GözE

F.

1803 seticornis

L.

Вон.

Sign. Germ. BoIsD. $\mathrm{F}$.

\section{STÅL}

$1803^{5}$ ) $\left(1758^{6}\right)$ $18+2^{6}$ ) $1781^{6}$ ) $1778^{6}$ ) $\left.1775^{6}\right)$ $\left.1758^{6}\right)$ $1852^{6}$ ) $\left.1879^{6}\right)$ 1833 bomairei ..... 1832 bipunctata .... 1837 unbila . puberula

1795 citri . RlleterHow. $1832^{6}$ ) $\left.1835^{4}\right)$ $\left.1775^{4}\right)$ $\left.1853^{4}\right)$ proletella 1878 radiata . L. Först. $\left.1893^{7}\right)$ A.r. \& SERv. 1803 calcaratus 1858 AM. \& SERT. J. Curt. 18.13 albidus .

SpIN.

LATR.

STÅL

STÅL

Anthocoris .... Aonidia

FALL. 1843 tristis 1825 albolineatum . Aphalara Aphamus Thlig. Först. LAP.

Aphelocheirns

WESTW. 1837 laevis ....... 1829 foliacea 1873 grossipes 1865 variegata plebeja. 1814 cruciata. 1869 nemortum Aphis

\section{8} 1832 alloacuminatus vulgaris .....

\begin{tabular}{c|c} 
L. & $\left.1755^{6}\right)$ \\
HAHN & $\left.181^{6}\right)$ \\
GEER & $\left.1773^{5}\right)$ \\
F. & $\left.1781^{6}\right)$ \\
F. & $\left.1775^{6}\right)$ \\
FIEB. & $\left.1851^{6}\right)$ \\
F. & $\left.1803^{5}\right)$ \\
F. & $\left.1803^{5}\right)$ \\
F. & $\left.1781^{5}\right)$ \\
THUNB. & $\left.1783^{4}\right)$ \\
VOLL & $1868^{4}$ \\
F. & $\left.1775^{4}\right)$ \\
L. & $\left.1761^{6}\right)$ \\
BotCHE & $\left.1833^{3}\right)$ \\
L. & $\left.1761^{1}\right)$ \\
L. & $\left.1758^{6}\right)$ \\
GöZE & $\left.1778^{6}\right)$ \\
SCHUL. & $\left.1829^{6}\right)$ \\
F. & $\left.1803^{6}\right)$ \\
L. & $1758^{\circ}$
\end{tabular}


Aphrophora... Geru.

Apodiphus .... Aradus .

Arma

Arytaena

Aspavia

Aspidiotus

Aspongopus ... Asterolecanium Athysanus .... Atractotomus. .

Aulacaspis .... Bagrada . . . . .

Bathycoelia ... A A. \& SERV.

Belostoma .... Latr.

Berytus

Blissus

Brachyplatys .. Brochymena... Ax. \& Serv.

BuRI.

BOISD.

Bythyscopus... Callidea

Gern.
Lap.

Calocoris

$$
\text { Fieb. }
$$

ANI. \& SERT

REUTER

REUTER

Fieb. An. \& SERv.

Cantao

Capsins

Carcinocoris...

Carpocoris

Centrotus .....

Ceresa F. HANDL. KOL. F. Ax. \& SERv.

Ceroplastes Grax Chermes
L.

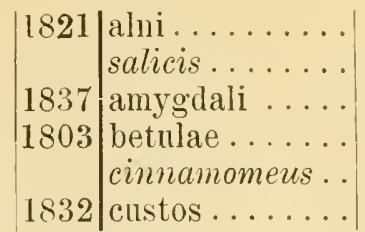

1848 genistae

1865 armigera

1833 perniciosus

britannicus destructor.

1832 jantus

1869

1838

1858

variolosum .

algentatus

mali

magn

1893

1862

pentagona

1

1843

1807

1803

1835

1835

$18 \pm 3$ picta

thalassina

grande

clavipes

lencopterus.

nigriventris

annulata.

obscura.

quadripustulata

1833 flavicollis

1832 bohemani

apicalis

1858

affinis

fulvomaculatus

norvegicus

1843

1896

lateralis

1878

pinastri

verbasci

1860 viroula .

1843

ocellatus.

1803

1897

1846

ater

cas

1803

1843

castetsi .

purpureipennis .

cornutus

borealis

1830 rusci

1758 abietis
FALL.

GeER

Gerur.

L.

Panz.

F.

LATh

F.

Const.

Newst.

Srgn.

H.

RAtzв.

F.

$1805^{6}$ )

$1773^{6}$ )

$\left.1817^{6}\right)$

$1758^{6}$ )

$\left.1794^{6}\right)$

$1794^{6}$ )

$1804^{1}$ )

$1781^{4}$ )

$1881^{3}$ )

$1896^{3}$ )

$\left.1869^{3}\right)$

$\left.1775^{4}\right)$

$1870^{3}$ )

$1794^{6}$ )

MEYER-DÜr. 18436)

FALI.

Targ.

Burn.

F.

H.-ScH.

L.

F.

SAY

WEsTW.

F.

H. $-\mathrm{ScH}$.

F.

L.

STÅL

Schout.

H.-ScH.

GeER

Gir.

Gern.

FALL.

$1807^{6}$ )

$188.5^{3}$ )

18354)

$\left.1775^{4}\right)$

$\left.1844^{4}\right)$

1758

$\left.1775^{6}\right)$

$\left.1831^{5}\right)$

$\left.1837^{4}\right)$

$\left.1775^{4}\right)$

$\left.1840^{4}\right)$

$\left.1775^{4}\right)$

$1758^{6}$ )

$\left.1853^{4}\right)$

$1903^{4}$ )

$\left.1835^{6}\right)$

$1773^{6}$ )

$\left.1788^{6}\right)$

$\left.1817^{6}\right)$

$\left.1807^{6}\right)$

MeYer-Dür. 184.36)

H.-ScH.

$1835^{6}$ )

Thunb.

$\left.1784^{4}\right)$

L.

HANDL.

$1758^{6}$ )

GEER

$1897^{8}$ )

\begin{tabular}{c|l} 
L. & $1758^{6}$ ) \\
F. & 1803 \\
FAIRII. & 1846 \\
L. & $1758^{3}$ ) \\
L. & 1758
\end{tabular}




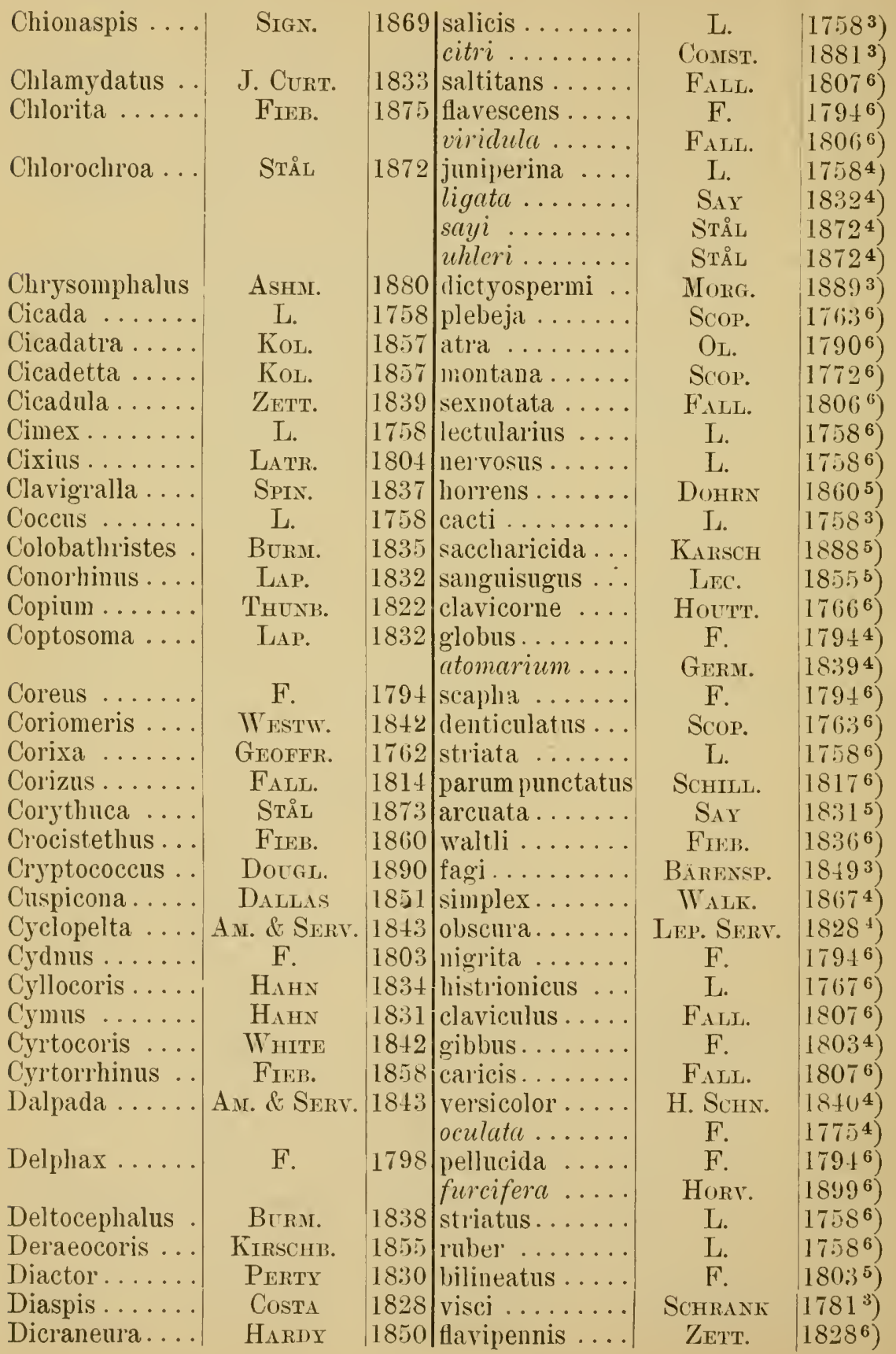


Dictyophara... Dicyplus

Dindymus.....

Dinidor.

Discocephala ..

Dolycoris .....

Dysdercus .... Am. \& SErv.

$\begin{array}{ll}\text { LAP. } & 1832 \\ \text { Muls. \& Rex } 1866 \\ \text { AM. \& SERv. } 1843\end{array}$

Edessa .......

Elasmostethus

Emesa

Empoasca

Entilia .

Epidiaspis

Eriococcus

Erythroneura..

Euacanthus ...

Eupelix .......

Euphyllura ...

Eupteryx .....

Eurydema

Eurygaster....

Euschistus ....

Eutettix

Euthyrhynchus

Filippia

Fiorinia

Flata

Fonscolombea

Frogattia ......

Fulgora .......

Gargara

Geocoris

Gerris.

Germ.

Fieb.

STÅL

LATR.

LAP.

\section{F.}

Fieb.

F.

WALSH

Am. \& Serv.

Cock.

TARr.

Fiтch

LEP. \& SERT. 1827

Gerir.

Först.

J. Curt.

LAP.

\section{LAP.}

Dallas

DUZEE

Dallas

TARg.

'TARG.

F.

LCHT.

Horv.

L.

Ayr. \& Serv.

FALL.

F.

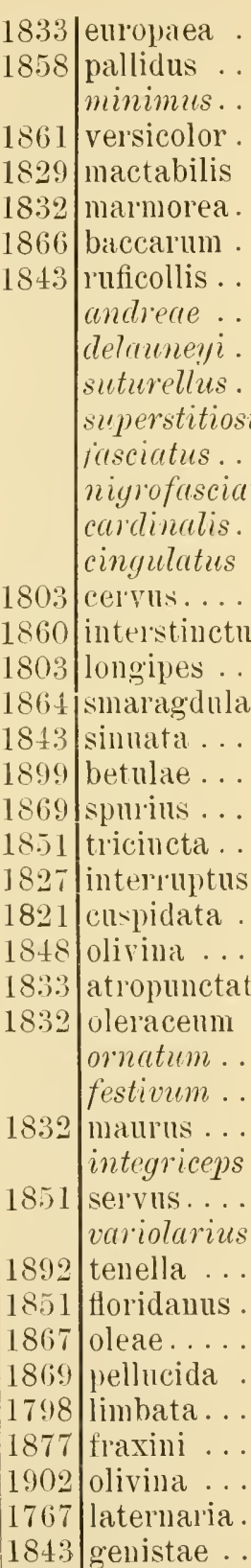

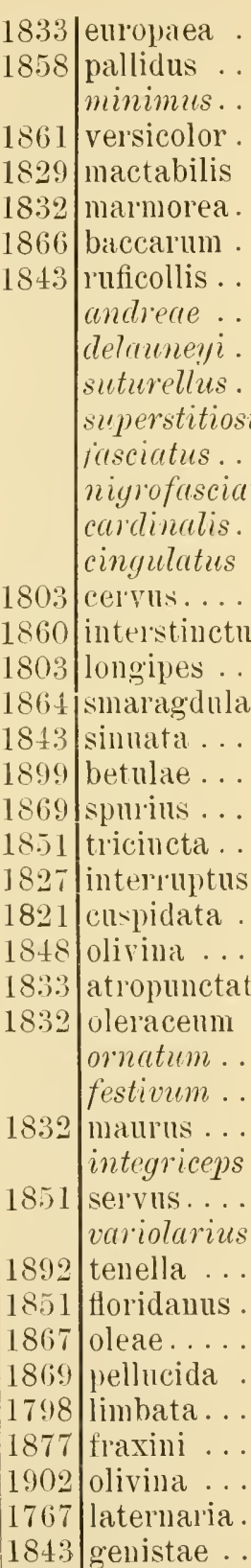

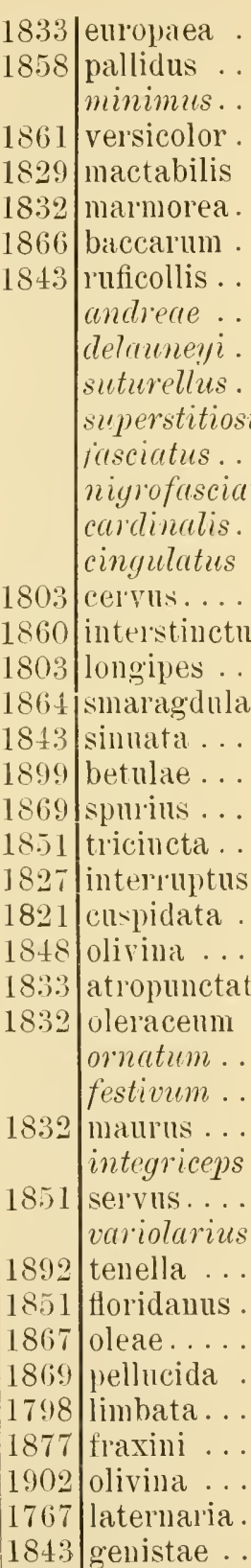

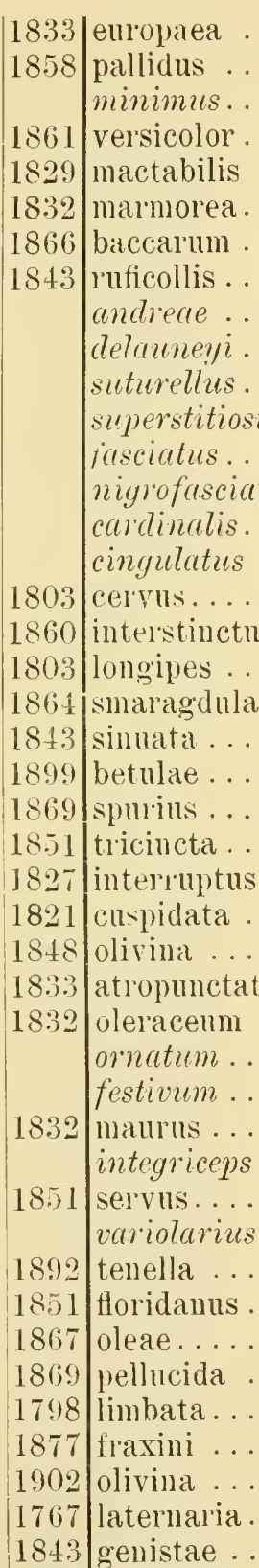

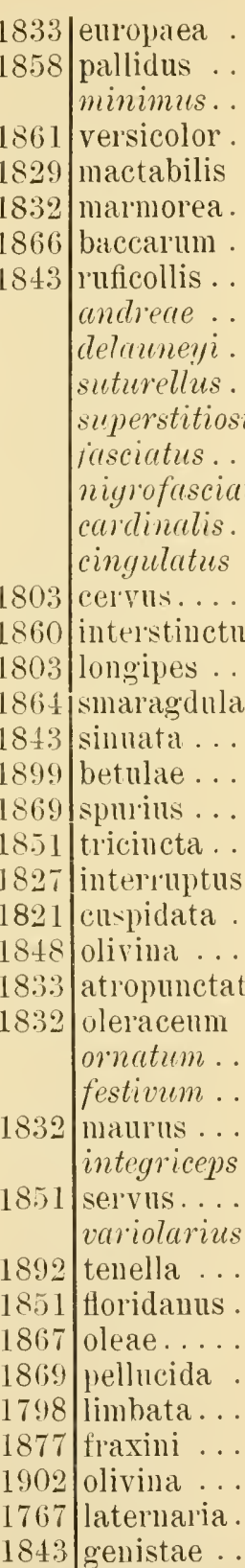

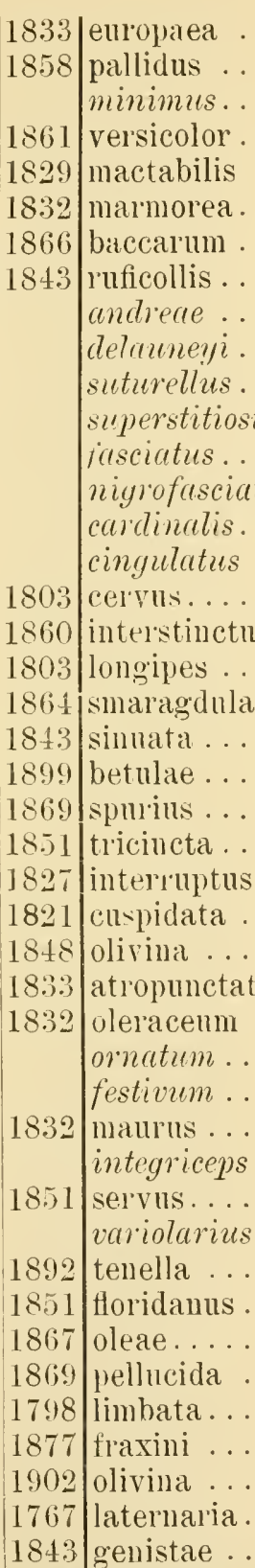

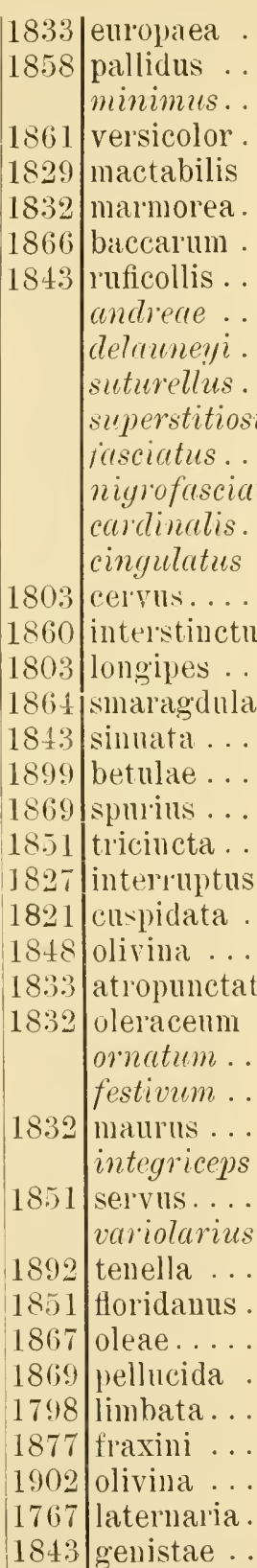

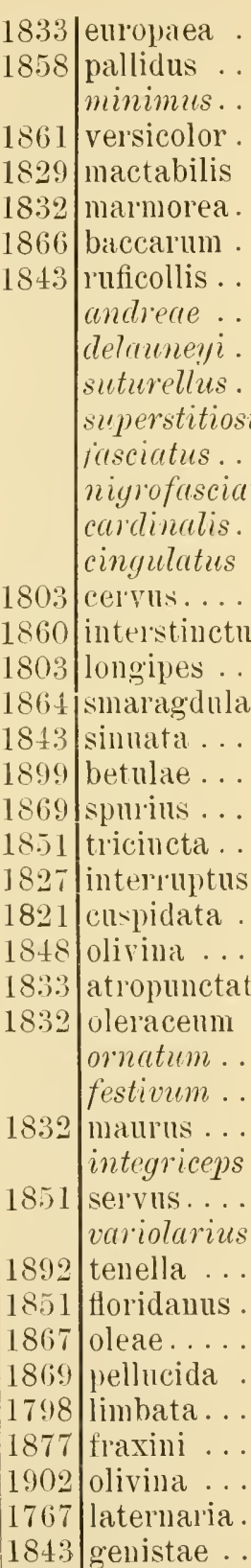

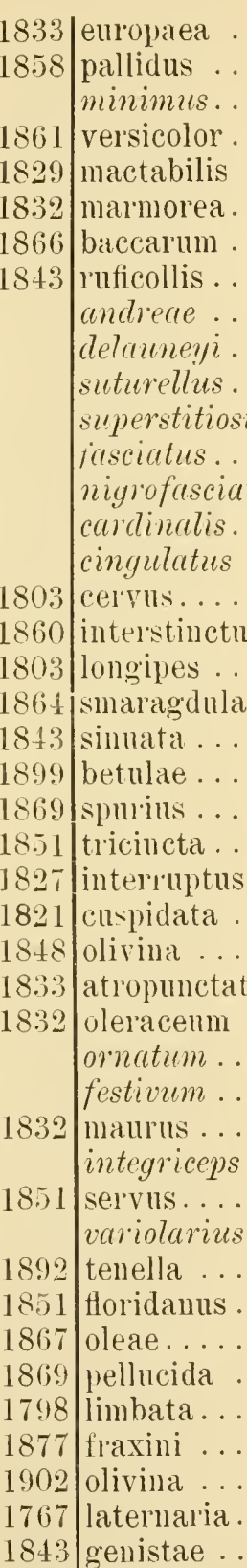

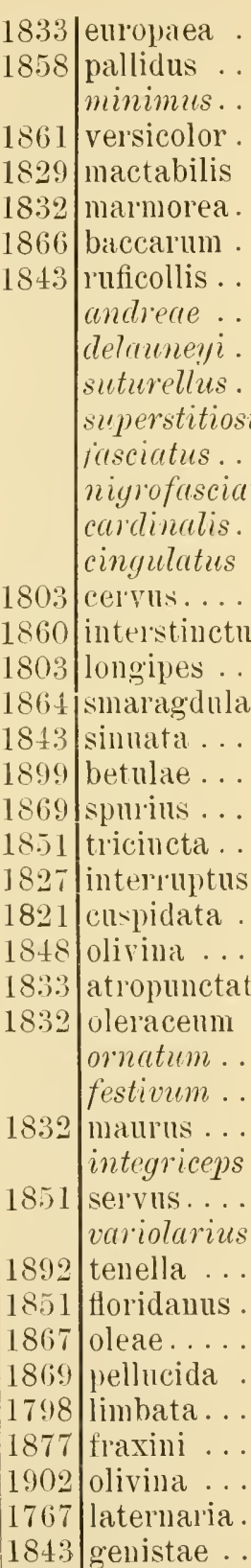

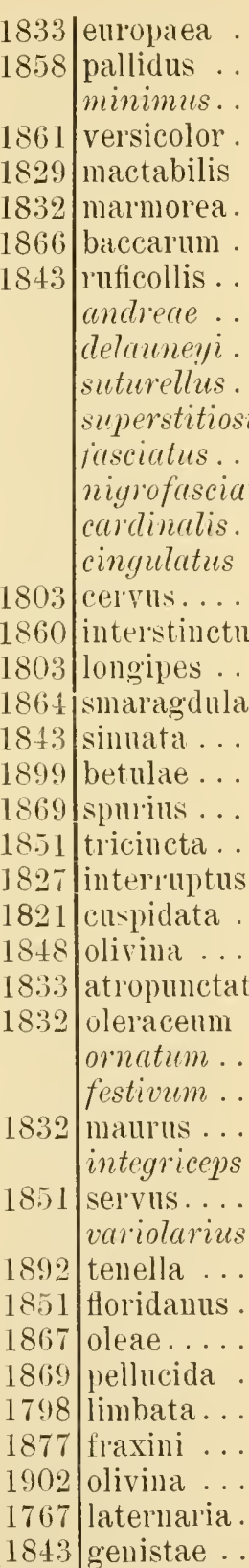

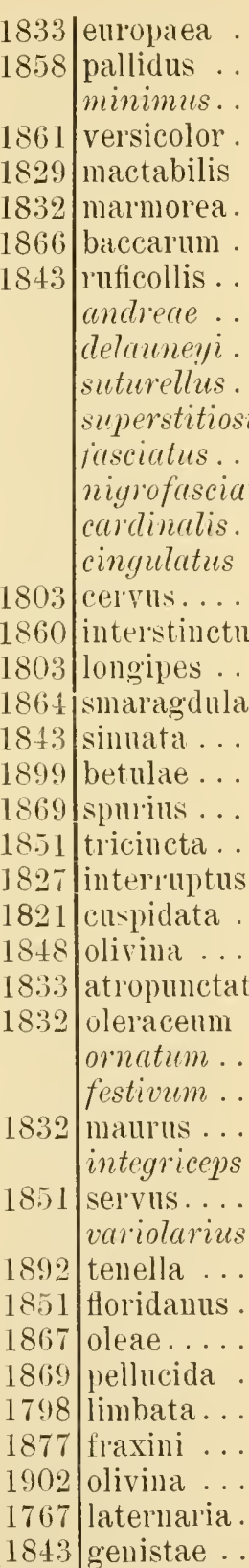

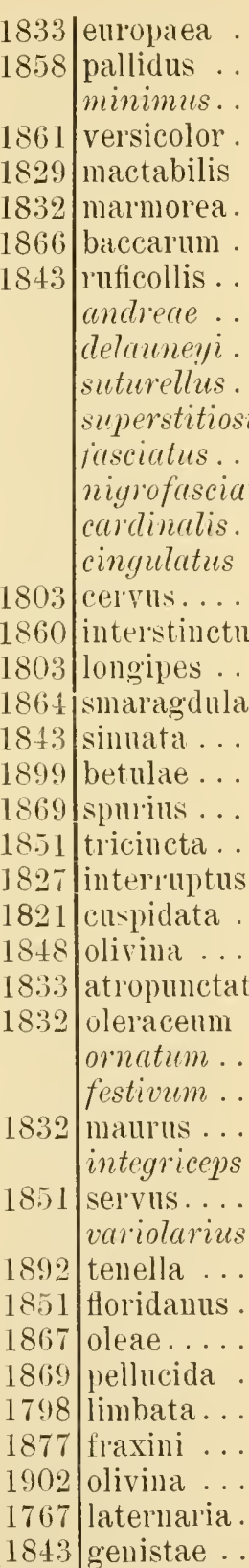

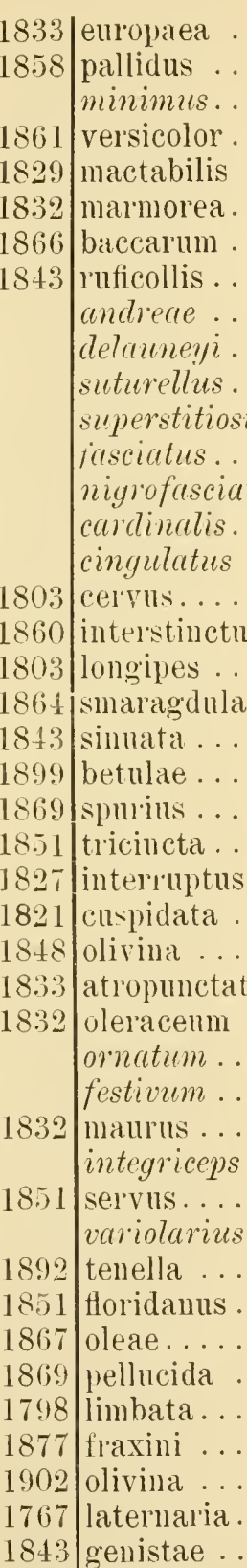

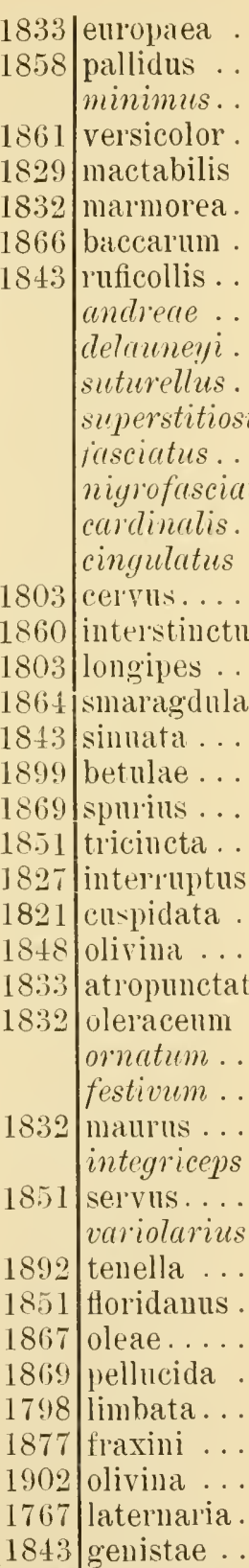

H.-ScH.

UHL.

H.-ScH.

Perty

LAP.

L.

L.

I.

LFTH.

H.-ScH.

F.

Sifin.

STÅL

Gerst.

F.

F.

L.

Geer

F.ILL.

F.

BäRENSP.

MoD.

Fiтch

L.

F.

O. Costa

Göze

L.

L.

I.

I.

Puton

SAY

Pall.

BAKER

L.

Costa

TARG.

F.

Kaltb.

Horv.

I.

F.

L.

I. $\left.1767^{6}\right)$

$\left.1835^{6}\right)$

1899

$\left.1853^{5}\right)$

$1833^{4}$ )

$1832^{4}$ )

$\left.1758^{6}\right)$

$\left.1764^{5}\right)$

$\left.1758^{5}\right)$

$\left.1881^{5}\right)$

$1842^{5}$ )

$\left.1775^{5}\right)$

$1860^{5}$ )

$1855^{5}$ )

$\left.1873^{5}\right)$

$1775^{5}$ )

$\left.1787^{4}\right)$

$1758^{6}$ )

$\left.1773^{5}\right)$

$1806^{6}$ )

1792

$\left.18 \pm 9^{3}\right)$

$1778^{3}$ )

1851

$\left.1758^{6}\right)$

$\left.1775^{6}\right)$

$1839^{1}$ )

$1778^{6}$ )

$\left.1758^{6}\right)$

$1758^{6}$ )

$\left.1767^{6}\right)$

$1758^{6}$ )

$\left.1881^{6}\right)$

$\left.1832^{4}\right)$

$\left.180.5^{4}\right)$

$\left.1767^{4}\right)$

$1828^{3}$ )

$\left.1869^{3}\right)$

1794

$\left.1874^{3}\right)$

1902

1758

$\left.1794^{6}\right)$

$\left.1761^{6}\right)$

$1794 \mid \begin{aligned} & \text { lacustris } \\ & 1814\end{aligned}$ 
Ghilianella.... DoHre 1860 servillei ..... Globiceps ..... Lep. \& SERv. 1825 flavomacnlatus . Gonocer'us .... Latr. 1825 acuteangulatus. Graphosoma... Idap.

Halobates.

Halticus

Halys

Hamamelistes

Harpactor

Hebrus

Helopeltis

Henestaris

Henicocephalus

Heterocordylus

Heterogaster . .

Holoptilus ... TuP. \& S

Eschz.

1822 sericeus

$\mathrm{H}_{\mathrm{AHN}}$

F.

SHIMIER

Spin.

J. Curt.

SIGN.

SpIN.

WESTW.

Fieb.

Schill.

829 urticae .......

Hormaphis ....

Howardia

Hyalopterus

Hydrometra. .

Hysteropterum

Icerya.......

Idiocerus

O.-S.

BERL. \&

\section{LEONARDI}

KосH

LATR.

AM. \& SERv.

SigN.

LEW.

Ischnaspis

Issus .

Ityraea

Jalla

Jassus.

Kermes.

Lachnus

Lecanium .

Ledra .

Lepidosaphes.

Leptocorisa ...

Leptoglossus

Leptopterna...

Lepyronia

Lencaspis .

Livia
DougL.

F.

STTÅ

HAHN

F.

BoIT.

Burar.

Buki.

F.

Shumer

LiATR.

GuER.

Finis.

Aur \& Serv.

'TARG.

LATR.
1867 betulae.

1837

annulatus

1833

1858

1837 halophilus

1837

1858

1829

1825

1861

1896

1854

1796

1843

1875

1834

biclavis

prouni

stagnorum

purchasi .

populi .

cedustus.

1887

longirostris

1803

coleoptratus

1866

1832

1803

1828

1835

1835

180:3

nigrocincta

dumosa.

atomarius

quercus.

exsiccator.

hesperidum

1868

1825

1858

184.3

1869 candida

1807 juncorım
SPIN.

F.

Reuter

Göze

MÜLL.

I.

F.

Eschz.

GEOFFR.

$\mathrm{F}$.

1803 dentatus ..... . . .

ursus .......

grylloides .

MoRDw.

Poda

L.

FALL.

WESTW.

$\left.1837^{5}\right)$

$\left.1794^{6}\right)$

$1879^{6}$ )

$1778^{6}$ )

$\left.1766^{6}\right)$

$\left.1758^{6}\right)$

$1775^{6}$ )

$1822^{6}$ )

$1785^{6}$ )

1775

1 1.01

$1761^{6}$ )

$1758^{6}$ )

$\left.1807^{6}\right)$

BurM. $1835^{6}$ )

W'ESTW. 1837

H.-ScH. $1835^{6}$ )

F.

$\left.1775^{6}\right)$

LEP. \& SERV. 1825

FITCH

1851

Const.

$1883^{3}$ )

Scop.

1763

L.

F.

MAsK.

L.

H.-ScH.

SIGN.

GEOFFR.

WALCK.

I.

$\left.1758^{6}\right)$

$1794^{6}$ )

$1878^{3}$ )

$\left.1758^{6}\right)$

$\left.1836^{6}\right)$

$1882^{3}$ )

$\left.1762^{6}\right)$

1858

F.

$1758^{6}$ )

L.

Altum

$1794^{6}$ )

$\left.1758^{3}\right)$

L.

1882

$\left.1758^{3}\right)$

aurita .......

Botché

ulmi ........

1836 membranaecus .
L.

L.

$\left.1758^{6}\right)$

$\left.18.51^{3}\right)$

$1758^{3}$ )

F.

$1803^{5}$ )

F.

$\left.1781^{5}\right)$

I.

$\left.1758^{6}\right)$

L.

$1758^{6}$ )

TARG.

$1869^{3}$ )

LATR. 
Lopus .

Lyctocoris

HAHN

1831 gothicus .

HAHN

1835 campestris

Lygaeus

F.

Lygidea

Reuter

1794 lencopter'us

Lygils .

$\mathrm{H}_{\mathrm{H} N \mathrm{~N}}$

Macrocorisa ...

Macrolophus

Thons.

1879 mendax

Macropsis .

Fieb.

1831 pratensis

Macrosiphum .

An. \& Serv.

Pass.

Menida

Mesovelia.

Мotsch.

M ULS.

KIRK.

Micronecta....

Microphysa

WESTW.

WESTW.

LEACH

KoCH

Fieb.

Microvelia ....

Mindarms

Miris

EP. \& SERY

Mollanthia

\section{STÅL}

Myodocha.

Myrmecoris

LATR.

Gorski

Myrmedobia

Bärensp.

Myrmus

HAHN

Pass.

LATR.

F.

LATR.

L.

MATS.

Nephotettix ...

Nezal'a

Air. \& Serv

L.

Notonecta

Nysins

Fieb.

DALIAS

Odontotarsus .

LAP.

Oliarius

Ommatidiotus

STÅL

Oncocephalus . .

Oncopeltus....

Orthezia ......

Orthotylus

Oxycarenus

Spin.

KLUG

STTÅL

Bose

Fieb.

Fieb.

18.58

pabulinus.

1869 geoffi'oyi.

1858 nubilis

1843 lanio.

1861

1852 furcata

1897

$18.3 \pm$ pselaphiformis .

1834 pyomaea . . . . .

1814

1857

piofana.

1858

1825

abietinus

1862

1807 serripes .

1852 gracilis

1857 col

1831 miriformis

1860 ribis

1802

1775

1804

1758

1902

$18 \pm 3$

1758

1858

1852

1832

vinitor.

imicoides

tipularius . .

cinelea

picalis.

viridula

glanca

erratica

thymi

purpureo -

lineatus

1862 pallens

1839

1830 squalidus

1868

1784

anlicus

1858

miticae

1836

nassatus .....

lavaterae

hyalinipennis

Palaeococcus

Cock.

1894 fuscipennis

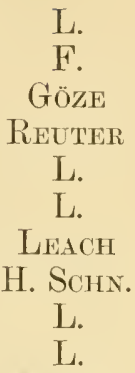

$\left.1758^{6}\right)$

$\left.1794^{6}\right)$

$\left.1778^{6}\right)$

1879

$1758^{6}$ )

$1761^{6}$ )

$1818^{6}$ )

$1835^{6}$ )

$\left.1758^{6}\right)$

$\left.1758^{6}\right)$

Motsch.

$\left.1861^{4}\right)$

Muls.

L.

J. Centr.

$\left.1852^{6}\right)$

$\left.1758^{6}\right)$

DuF.

F.

Кं०CH

L.

Sohrank

$\left.1833^{6}\right)$

$1833^{6}$ )

$\left.1803^{5}\right)$

1852

$\left.1758^{6}\right)$

$1782^{6}$ )

HAHN

OL.

$\left.1834^{4}\right)$

$\left.1811^{5}\right)$

Sahlibg.

FALL.

FALL.

L.

L.

I.

I.

L.

Mo'tseh.

$\left.18+8^{6}\right)$

$1807^{6}$ )

$\left.1807^{6}\right)$

1758

$\left.1758^{6}\right)$

$\left.1758^{6}\right)$

$1758^{6}$ )

$1758^{6}$ )

$1859^{6}$ )

L.

I.

I.

WOLFF

$\left.1758^{6}\right)$

$1758^{6}$ )

$\left.1758^{6}\right)$

$1804^{6}$ )

BERGR.

Rossi $1790^{6}$ )

GERMI. 18216)

FALL. $1806^{6}$ )

RossI

F.

L.

F.

F.

$\left.1790^{6}\right)$

$\left.1775^{5}\right)$

$\left.1766^{3}\right)$

$\left.1787^{6}\right)$

Costa

$\left.1787^{6}\right)$

Burm. 
Palomena ..... Muls. \& Rey 1866 prasina .......

Parlatoria .... TARg.

Pediopsis

Pelogonus

Pempligus

Pentatoma

Pentlimia

Perkinsiella .

Pliantia

Pliellacoccus

Plilaenus ..... STÅ

Phloeal ....... Le Lep. \& Serr.

Phorodon

Phylliphis

Phyllomorpha .

Phyllontocheila

Phylloxera

Plymata

Physatochila

Pliytocoris

Picromerus....

Piesma

Piezodorus

Pilophorus

Pineus

Pinnaspis .....

Pirates

Plagiognathus

Plataspis

Platypleura

Plea

Plesiocoris

Ploiaria

Ploiariola

Poecilocoris ...

Pollinia

Porphyrophora

Psacasta......

Psallus

Psendococcus . .

Psendoparlatoria

Psylla.

Psyllopsis
Pass.

KOCH

LAP.

Puton

Fonsc.

LATR.

Firb.

Fall.

An. \& Serv. 1843 LEP. \& SERT. 1825

FIEIs.

IVESTW.

ShIMIER

Cock.

Serr.

Fieb.

STÅL

A M. \& SERY.

LEACH

Fieb.

Srop.

Rectrer

Dalulas

Thise.

Branit

Geris.

Fieb.

WESTW.

Cock.

GrofFr.

F. Löw 1839 1869 189.2 18.31 1858 1865 1817 1861 1786 1888 1848 1869 1833 1839
1860 1843 1839 exanthematica . 1858 pergandel blanchard . ii... marginatus. bursarius rufipes

nigra ... -.... 1789 1903 saccharicida. . subquadrata... aceris . spumarius corticata liumili . fagi laciniata cardui . vastatrix quercus. crassipes quadrimacuiata populi ........ bidens. capitata lituratus...... clavatus ...... strobi . aspidistrae hybridus. . . arbustorum coccinelloides . stridula

minutissima... rugicollis domestica.... vagabunda druraei pollini . polonica variabilis ..... citri

1892 parlatorioides. 1762 alni .

1878 fraxinicola
L.

Const.

TARG.

F.

LATR.

L.

I

GöZE

KiRK.

H. Schx. SII.

FALL.

DrERT

SchraNk

L.

VILL.

L.

Planchon

Foxse.

F.

WOLFF

L.

L.

IT OLFF

F.

L.

Hartig

SIGX.

Scop.

F.

LAP.

L.

Fuessiy

FAlL.

Scop.

L.

I.

Costa

I.

Scop.

FALL.

Risso

Const.

L.

Fölist.
$1761^{6}$ ) $1881^{3}$ ) $\left.1869^{3}\right)$ $\left.1794^{6}\right)$ $1807^{6}$ )

1758

$\left.17: 8^{6}\right)$

$\left.1778^{6}\right)$

190.3

$\left.1837^{6}\right)$

$1880^{3}$ )

$\left.1826^{6}\right)$

$\left.1773^{4}\right)$

781

1767

$\left.1789^{6}\right)$

$\left.1758^{6}\right)$

1868

18.31

$\left.1775^{6}\right)$

$\left.180 \div^{6}\right)$

$\left.17.8^{6}\right)$

$\left.17: 8^{6}\right)$

$1804^{6}$ )

$\left.179 \pm^{6}\right)$

$\left.1767^{6}\right)$

$\left.1869^{3}\right)$

$\left.176.3^{6}\right)$

$\left.1794^{6}\right)$

$1832^{4}$ )

1758

$17756)$

$1807^{6}$ )

$\left.1786^{6}\right)$

$1758^{6}$ )

$\left.1771^{4}\right)$

$\left.1828^{3}\right)$

$\left.1758^{3}\right)$

$17\left(0: 3^{6}\right)$

$1829^{6}$ )

$\left.181: 3^{3}\right)$

$\left.1883^{3}\right)$

$1758^{1}$ )

$\left.1848^{1}\right)$ 
Ptilocnemus ...

Pulvinaria

WESTW.

Pycanlum

Pyrrhocoris

Rallatia

Reduvius

Rliaphigaster . .

Rlinocola.

Rhoeocoris ....

Rhopalosiphum

Rhopalus .....

Ricania.

Ripersia

Riptortus

Sallbergiella.

Saldil

Schizoneura ...

Sciocoris .

Scolopostethus .

Scutellera

Scutiphora

Selin'us .

Spliaerococcus

Spluaerocoris

Spilostethus

Stenocephaius

Stenotus

Stictocephala.

Strachia

Syromastes.

Systellonotus

Tectocoris

Tenthecoris

'Tesseratoma.

Tetraneura.

Tettigia.

'Tettigometra .

'T'ettigonia

Thamnotettix. .

'Therapha.

'T'byantlıa.

Thyreocoris

Tibicina
TARG.

Air. \& SERT.

FALL.

F.

F.

LAP.

Först.

Bergh.

Pass.

FiEB.

Geru.

SigN.

STAL

HAGL.

F.

Hantig

FALL.

Fieb.

LAI.

LAP.

An. \& SERY.

MAsK.

Btrat.

STAL

IATR.

J Ak.

STÅL

HAHN

LATR.

FIEI.

HAHN

ScotT

BERTHOLd

HARTig:

Kú.

LATR.

GeOFFr.

(nec. L.)

ZETTS.

A II. \& SERT.

STÅL

Schrank

Kon.
$1840 \mid$ lemur

1869 betulae

1843 rubens

1814 apter'us

1790 linearis

1775 personatus

1832 nebulosa.

1848 aceris

1895 sulciventris

1860 lactncae

1861 crassicornis

1818 fenestrata

1875 falcifera

185y linearis .

1895 sinuularis

$180:$ saltatoria

1840 humigera

1829 cursitans

1860

1860

1832 pedicillata

1843 bicolor

1891 marlatti

1835 ammulus

1868 equestris

1825 agilis

1877 binotatus

1869 inermis .

1833 crucigera

1825

18.58 triguttatus

1834 lineola

1886 bicolor

1827

1841

papillosa.

18.7

1804

ulmi

1798

or'ni .

bliqua

vilidis.

1830

(1)

1843 liyoscyami

1862

custator.

perditor.

1801

scarabaeoides. .

pulicarius

1857 haematodes
W Histw.

L.

F.

L.

L.

I.

Poda

L.

STÅL

Kantb.

L.

F.

KÜNCK.

F.

HAGL.

L.

Hausm.

F.

Schili.

$\mathrm{F}$.

Kinby

L.

CoCK.

F.

I.

Scop.

F.

F.

HАHN

L.

L.

F.

Scot'T

Drers

Geien

L.

Panz.

L.

FALL.

I.

F.

F.

L.

$1840^{5}$ )

$1758^{3}$ )

$\left.1794^{4}\right)$

$\left.1758^{6}\right)$

$\left.1758^{6}\right)$

$1758^{6}$ )

$1761^{6}$ )

$\left.1758^{1}\right)$

1863

1843

$1758^{6}$ )

1803

$\left.1878^{3}\right)$

$1775^{5}$ )

1895

$1758^{6}$ )

1802

$1794^{6}$ )

$1829^{6}$ )

$1775^{4}$ )

$1826^{4}$ )

$1758^{6}$ )

$1899^{3}$ )

$\left.1775^{4}\right)$

$\left.1758^{6}\right)$

$\left.1763^{6}\right)$

$\left.1794^{6}\right)$

1775

$18.33^{4}$ )

$\left.1758^{6}\right)$

$\left.1767^{6}\right)$

$\left.1781^{4}\right)$

1886

$\left.1770^{4}\right)$

1773

$\left.1758^{6}\right)$

$\left.1799^{6}\right)$

$1758^{6}$ )

GERM.

$\left.1826^{6}\right)$

$1758^{6}$ )

$1803^{4}$ )

$\left.1794^{4}\right)$

$\left.1758^{6}\right)$

$1839^{2}$ )

Scop. 
Tingis

F.

1803 pyri

Ay. \& Serv. 1843 furcata .

F.

$\left.1775^{6}\right)$

Tomaspis ..... An. \& SE

Trama ....... Herdex

1852 graminum

GERM.

$\left.1821^{9}\right)$

Triatoma

LAP.

1837 t

RoND.

Hetien 1837

Triecphora... Aar. \& SERv. 1843 sanguinolenta. .

Trigonotylus .. F. Fев.

1858 ruficol'nis .....

Trioza ....... Först.

1848 urticae .......

Triphleps ..... F. Fisв.

Troilus

\section{STÅL}

1860 minuta

Typhlocyba ...

Ulopa ...

Tacuna......

Geris.

1867 lmidus

KL.

L.

$\left.1834^{5}\right)$

GEOFFR. $\quad 1785^{6}$ )

L.

$\left.1758^{1}\right)$

L. $1758^{6}$ )

F. $\left.1775^{6}\right)$

Fitl. 1814 reticulata....

Velia ....... Litk.

Verlusia..... Sp Spr.

1837 dryophila ....

L.

$\left.1758^{6}\right)$

F.

SChrate

$\left.1794^{6}\right)$

Heye ex

1804

F.

F.

1801

L.

$\left.1794^{6}\right)$

$\left.1775^{6}\right)$

1837 rhombea.....

Lit.: 1) Aulmaxn. P'syllidarum Cat., Berlin 1913.

2) Basks, Uat. nearetic Hemipt.-Heteropt., 1910.

3) F'ernald, Cat. Coccilae, 1903.

4) Kirkatdy, Cat. Hemipt.-Heteropt., Berlin 1909.

5) Lethierky-Severix, Cat. gén Hlemipt., 1893-189t.

6) Osfanis, Verz. paläarkt. Hemipt., 1906-1910.

7) Quaintance in: (ien. Ins., fase 871908.

8) Haxplnksch in: Aun Hofmus. Wient, v. 121897.

9) Lalleasand in: Gen. Ins., fase. 14:3 1912.

Schumacher (Charlottenburg).

Anoplura.

Haematopinus .

Pediculus

Phthirius .... Ger.
LEACH

L.

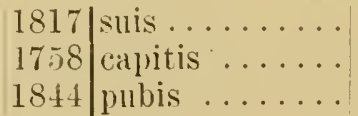

\begin{tabular}{l|l|l|}
1817 & suis . . . . . . \\
1758 & capitis . . . . \\
1844 & pubis . . . .
\end{tabular}

\section{Mallophaga.}

Colpocephahm.

Docophorus ...

Goniocotes....

Gyropus .......

Lipeur'us

Menopon......

Nirmuls

NITZ:CH

NITZNCH

Butrit.

NIT\%:CH

NITZSCH

NITZsel

NITZSCHI

Trichodectes .. NIrzsch
1818 flatrescens..... N. Nitzsch 1818 1818 communis .... N N 18.35 compar ...... NITzsch 1818 1818 oralis ....... Nitzso 1818 1818 variabilis ..... N. Nт̈зон 1818 1818 pallidum....... Nitzsch 1818 1818 fuscus . . . . . . . NITZSC H

(Grebel) 1814 NITZNCH 1818

Lit.: Kelloga in: (ren. Ins., fise 661908.

Densy, Monogr Anopl. Britan., 1812. 


\section{Diptera.}

\begin{tabular}{|c|c|c|c|c|c|}
\hline Acrocera ..... & MEIG. & $|1803|$ & globulus ..... . & Panz. & 1803 \\
\hline Anopheles .... & MEIG. & 1818 & bifucatus ... & L. & 1758 \\
\hline Anthomyia.... & MEIG. & 1803 & pluvialis ...... & L. & 1758 \\
\hline Anthiax ..... & F. & 1794 & nolíl $0 \ldots \ldots$ & I. & 1758 \\
\hline Asilus ........ & L. & $17 \div 8$ & crabr oniformis . & I. & 1758 \\
\hline Atherix ..... & MeIG. & 1803 & ibis ......... & F. & 1798 \\
\hline Beris . . . . . & LATR. & 1802 & clavipes ...... & $\mathrm{L}$. & 1767 \\
\hline Bibio ...... & GEOFFR. & 1764 & hol'tulanms .... & L. & 1758 \\
\hline Bombylius .... & L. & 1758 & major ........ & L. & 1758 \\
\hline Borborus ..... & MEIG. & 1803 & equinus ........ & FaLL. & 820 \\
\hline Caliiplıor"a .... & R. D. & $18: 30$ & vomitoria .... & L. & 1758 \\
\hline Calobata . . . . . & M eIG. & 1803 & petronella.... & L. & 758 \\
\hline Cecidomyia.... & MEIG. & 180.3 & pini .......... & GEER & 1782 \\
\hline Ceratopogon ... & MEIG. & 1803 & bipunctatus . & I. & 1767 \\
\hline Chilosia ..... & $M_{\mathrm{EIG}}$. & 1822 & oestracea .... & I. & 58 \\
\hline Chironomus ... & MEIG. & 1803 & plumosus $\ldots$. & L. & 758 \\
\hline Chlorops..... & MEIG. & 1803 & lineatus ...... & F. & 791 \\
\hline Chrysogaster . . & MeIG. & 1803 & coemeterior'm . & L. & 758 \\
\hline Chrysops .... & MFIG. & 1803 & coecutiens .... & L. & 758 \\
\hline Chrysotoximm . . & МEIG. & $180: 3$ & arcuatum .... & L. & $17: 8$ \\
\hline Clinocera ..... & MeIG. & 1803 & nigrad . . . . . . & MEIG. & 804 \\
\hline Clitellaria. . . . . & MEIG. & 1803 & epphippim ... & $\mathrm{F}$ & 781 \\
\hline Conops ....... & L. & 1761 & vesicularis ... & L. & 1761 \\
\hline Cordyla ..... & MeIG. & 1803 & fusca . . . . . . . & MEIG. & 1804 \\
\hline Corethra..... & MEIG. & 1803 & culiciformis ... & GEER & 1776 \\
\hline Ctenophora ... & Meig. & $180: 3$ & pectinicornis & L. & 758 \\
\hline Culex ....... & L. & 1758 & pipiens . . . . . & L. & $7 \div 8$ \\
\hline Dasypogon ... & MeIG. & 1803 & tentonus.... & L. & 1767 \\
\hline Dilophus..... & MeIG. & 1803 & vulgalis ... & MeIG. & 1818 \\
\hline Dioctria ..... & MeIG. & 1803 & oelandica .... & I. & 758 \\
\hline Dolichopus ... & LATR. & 1796 & ulıgulatus . . . . & L. & 158 \\
\hline Doros ....... & MeIG. & 1803 & conopseus... & F. & 775 \\
\hline Empis...... . & L. & 1758 & borealis ... & L. & 1758 \\
\hline Erioptera . . . . & MEIG. & 1803 & flavescens . . . & L. & 1758 \\
\hline Eriothrix . . . . & Mètg. & 1803 & rufomar'ulatus & GEFR. & 1776 \\
\hline Eristalis . . . . . & LAтR. & 1804 & arbustorum ... & I. & \\
\hline Emmerus..... . & MEIT. & 1822 & tricolor . . . . . . & Meit. & 1822 \\
\hline Exorista ...... & MEIG. & 1803 & larvarum ..... & L. & 1758 \\
\hline Gastrophilus . & LеACH & 1817 & lua emor rhoidalis & L. & 1758 \\
\hline Gonia ....... & МЕIG. & 1803 & fasciata ..... & MEIG. & 1826 \\
\hline Gymnosoma . . & MFIG. & 180.3 & I'otundatum ... & I. & 17 \\
\hline Haematopota. & MEIG. & 1803 & pluvialis ...... & L. & 17 \\
\hline Helophilus . . . . & MEIG. & 1803 & pendulus. . . . . . & L. & \\
\hline Heptatoma.... & Meig. & 1803 & pellucens ..... & F. & 177 \\
\hline
\end{tabular}


Hippobosea ...

Hybos...

Hypoderma

Laphria .

Leptis.

Leptogaster ...

Leucostoma ...

Limonia

Limosina

Lonchoptera .

Loxocera .....

Lucilia .

Macrocer'a ....

Melanopliora . .

Merodon......

Metopia .....

Microdon .....

Micropeza

Musca.

Mycetopliila .

Myopa

Nemotelus

Nephrotoma . .

Ochithera

Ocypter'x

Odontomyia.

Oestrus.

Oncodes

Oxycel'd

Pachygastel

Pangonia

Pedicia .

Penthetriat ....

Pliasia

Phor'a

Phthiriit

Pipunculus....

Platypeza.

Platystoma

Platýura. . .

Pollenia

Psila

Psichoda

Ptychoptera...

Pyrellia

Rlingia
L.

MIEIG.

LATr.

MEIG. F.

MEIS.

MEEIti.

ME:IG.

NACQ.

MeIg.

IIEIG.

R. D.

NEIG.

MeIG.

NEIG.

MEIG.

MIEIG.

IIIEIt.

L.

MIEIG.

F.

Geufrr.

IIEIG.

LATti.

Latri.

Mrig.

L.

LATR.

Mieig.

IIEIG.

IATti.

LaTR.

Mliti.

LATR.

Latr.

MI Lifi.

LATr.

MI:ITi.

Ml Liti.

II litis.

R. D.

MIsis.

LATTi.

IIsigi.

R. D.

Scup.

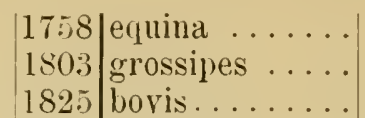

180:3 gibbosa......

180.5 scolopacea

180:3 tipuloides . .

180:3 a terrimum

1803 quadrimaculata

1835 pusilla

1803 lutea.

1803 ichnemmonea

1830 caesar.

1803 luteal.

1803 roralis

1803 equestris

180:3 lencocephala

180.3 mutabilis .

$18(13.3$ corrigiolata

1758 domestica.

1803 punctati.

1775 testacea

1764 pantherimus

180.3 dorsalis

180. mantis

1802 brassicaria

1803 hydroleon .

1758

1796 gibbosus.

1803 hiypoleon

1803 at $+\mathrm{H}^{\circ}$

1802

1809

180:3

1802

1796

180 ?

1802

1803

180:3:

ladistellata....

rivosa........

1803

1830

180:3 fime

1796 phalaenoides

1803 contaminata

1830 cada verina .

1763 rostrata
L.

1758

L.

1767

L.

1758

I.

1758

L.

1758

L.

1758

VILL. 1769

L.

1761

Meig. 1830

Paxz.

1809

L.

1758

L.

1758

MEIG.

1804

I.

F.

$17: 8$

Rossi

L.

1794

I

I. 1767

Meig.

1804

L.

1759

L.

1758

F.

1781

Geer

1782

F.

1775

L.

1758

L.

1761

L.

1758

L. $\quad 1767$

Paxz.

1798

F.

1781

L.

1758

MEIG.

1818

F.

17 ()!

MIEIT.

1804

MIKix 1796

LATR. $\quad 1804$

MeIr. 1804

L. 1761

MIEIT. 1804

F. $\quad 1786$

L. 1758

L. $\quad 1758$

L. 11758

L. $\quad 1758$

L. $\quad 1758$ 


\begin{tabular}{|c|c|c|c|c|c|}
\hline Rhyphiss...... & LATR. & $\mid 1804$ & fenestralis .... & Scop. & 1763 \\
\hline Sarcophaga ... & МeIG. & $18 \div 6$ & carnaria ...... & L. & 1758 \\
\hline Sargus ....... & F. & 1798 & cuprarius .... . & L. & 1758 \\
\hline Scatophaga ... & MEIG. & 1803 & scybalaria .... & $\mathrm{L}$. & 1758 \\
\hline Scatopse..... & GEOFFR. & 1764 & notata....... & L. & 1758 \\
\hline Scenopinus .... & LATR. & 1802 & fenestralis .... & L. & 1758 \\
\hline Sciara ...... & MEIG. & 1803 & thomae ...... & L. & 1767 \\
\hline Sericomyia .... & MEIG. & 1803 & lappona ...... & L. & 1758 \\
\hline Sicus . . . . . . . & Scop. & 1763 & ferrugineus ... & L. & 17 \\
\hline Simulium ..... & LATR. & 1803 & reptans....... & L. & 175 \\
\hline Siphona ..... & NeIG. & 1803 & irrritans ....... & L. & 17. \\
\hline Spilomyia . . . . & MEIG. & 1803 & diophthalma... & L. & 758 \\
\hline Stomoxys ..... & GEOFFR. & 1764 & calcitrans ..... & L. & \\
\hline Stratiomys .... & GEOFFR. & 1764 & chamaeleon ... & L. & \\
\hline Syritta...... & SERV. & 1825 & pipiens ....... & L. & 17 \\
\hline Syrphus ...... & F. & 1775 & ribesii . . . . . . & L. & 175 \\
\hline Tabanus ...... & L. & 1758 & bovinus . . . . . . & L. & 1 \\
\hline Tachina ...... & Мeig. & 1803 & grossa..... & L. & \\
\hline Tachydromia . . & MEIG. & $180: 3$ & cursitans ... & $\mathrm{F}$. & 17 \\
\hline Tanypus . . . . . . & MeIG. & 1803 & monilis ....... & L. & 17 \\
\hline Thereva...... & LATR. & 1796 & plebeja...... & L. & \\
\hline Tipula........ & L. & 1758 & lumata..... & L. & \\
\hline Toxophora .... & MeItr. & 1803 & maculata .. & Rossi & 17 \\
\hline Trichocera .... & MEIG. & 1803 & hiemalis ... & GEER & 17 \\
\hline Trinemra...... & MeIG. & 1803 & aterrima.... & F. & \\
\hline Trypeta ..... & MeIG. & 180.3 & florescentiae... & L. & \\
\hline Tolucella ..... & GEOFFR. & 1764 & bombylans .... & L. & 17 \\
\hline Xylophag'us ... & MEIG. & & cinctus ...... & GEER & 17 \\
\hline Xvlota ........ & MIEIG. & 1822 & segnis . . . . . . . & L. & 17 \\
\hline
\end{tabular}

Lit.: IIetgen, Germ. IIag., v. 21803.

Becker, Bezzi, Kertész, S'trein, Kat. paläarkt. Dipt., v. 1-4 1903-1907.

GrünBerg (Berlin).

Aphaniptera.

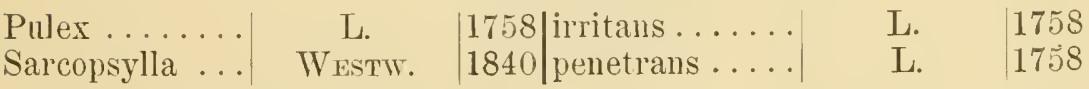
Lit.: Taschenberg, Flöhe, 1880.

Lepidoptera.

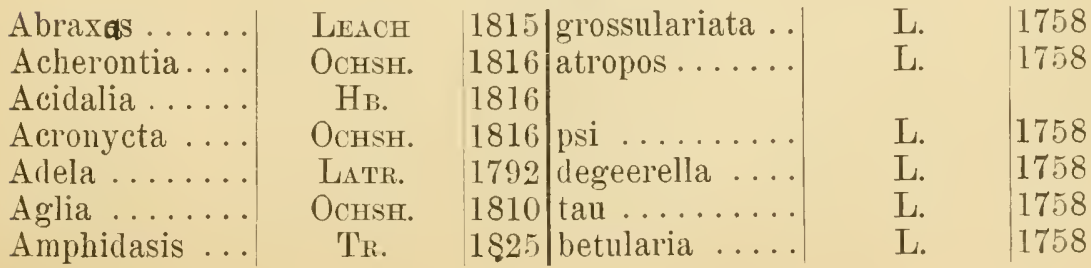


Amphipyra... .

Apatura ......

Aporia .......

Arctia.

Argynnis

Biston .....

Boarmia

Bombyx .....

Brephos

Callimorpha...

Caradrina.

Carpocapsa

Catocala......

Charaeds

Cheimatobia.

Coenonympha

Coleophora...

Colias

Cossus

Crambus

Cucullia .

Danais

Daphnis

Dasychila

Deilephila

Dilina.

Drepana

Endromis .

Epinephele.

Erebia

Fidonia

Fumea

Galleria

Gastropacha .

Gelechia......

Geometra....

Gnophos......

Hadena.

Hepialus.

Hesperia

Hibernia

Hypena

Hyponomeuta

Lno ...... .

Lasiocampa
OCHSH.

$\mathrm{F}$.

$\mathrm{HB}$.

SCHRANE

F.

LEACH

Tr.

L.

Ochsh.

LATR.

Нв.

Tr.

Schrank

STEPH.

Steph.

HB.

$\mathrm{HB}$.

F.

F.

F.

SchratK

Latr.

$\mathrm{HB}$.

STEPH.

Ochsh.

Datir.

SCIIRANE

OCHSH.

Нв.

DALM.

'T'R.

STEPH.

F.

OcHsh.

Нв.

L.

Tr.

SchrdNk

F.

F.

LAT'R.

Schrank

Sonoff.

LEACH

T'R.

Schrank
1816|

1807 il'is

L.

1758

1816 crataegi

L.

1758

1801 caja.

1807 paphia

L.

1758

L.

1758

181 j hirtaria

Clenck 1759

1825

1758 mori

L.

1758

1816 parthenias

L.

1761

L. $\quad 1758$

1822

1829 pomonella....

L.

1758

1802

L.

1758

1829

1829

glaminis . . . . .

bl'umata .....

L.

1758

L.

1758

I.

1758

F.

1787

F. $\quad 1794$

1794 ligniperda

1798

1801

1819 chrysippns ....

1822

1829 nerii.

1816

1816

pudibunda ....

1802

euphorbiae....

tiliale.

1810

1816

falcatania

1816

1825

rersicolora ....

janira . . . . . .

medusa

1829

1798

limbaria ......

casta

L.

1758

L. $\quad 1758$

L. $\quad 1758$

L. $\quad 1758$

L. $\quad 1758$

L. $\quad 1758$

L. $\quad 1761$

L. $\quad 17 \overline{8}$

L. $\quad 1758$

F. $\quad 1787$

F.

1775

PALL. 1767

L. $\quad 1758$

L. $\quad 1758$

L.

1758

1767

1827

1802

1775 hunผ

1793 malvae

1825 defoliaria .....

1802

1837 eronymellus ...

1815 statices.

L.

1758

1828

1802 quel cus

L.

1758

L.

1758 
Leucania

Lellcophasia

Liby thea

Limenitis

Lithocolletis

Lithosia ...

Lophopteryx .

Lycaena

Macroglossa...

Mamestra .....

Mania .

Melitaea ......

Nepticula .....

Neptis

Nola

Notodonta

Odonestis .....

Orgyia

Papilio

Pararge ......

Palluassius

Phalera

Pieris

Plnsia

Polia.

Psodos

Pterophorus...

Pyralis

Saturuia

Satyrus .

Scoliopteryx...

Sesia .

Smerinthus....

Sphinx

Spilosoma

Syntomis

Thecla

Thyris

Tinea

Tortrix

Trochilium.

Vanessa

Xanthia

Zeuzera

Hв.

S'TEPH.

F.

F.

Hв.

F.

1818

STEPH.

F.

Scop.

Нв.

T'r.

F.

HEID.

F.

LEACH

OCHSH.

Gerir.

OCHSH.

L.

$\mathrm{H}$.

LATR.

H.B.

Schrank

Ochsh.

Ochsн. \& Tr. 1816

1807 celtis

1807 populi

1818

1798

1828

1807 argus

1776

1822

1825

1807

stellatal'um

$18+3$

1807

1815

1810 ziczac ........

1812 pruni

1810

1758 podalirius

1816

1805 apollo

1822 bucephala

1801 brassicae

1816

$\mathrm{T}_{\mathrm{R}}$.

1828

GroFrr.

1762

L.

1767 farinalis

Schrank

1802 pavonia

LATR.

1810 briseis .

Germ.

1811

F.

LATR.

L.

1775

1802 populi .

1758 ligustri

STEPH.

1828

Ochsh,

F.

Ochsh.

L.

I.

Scop.

F.

Ochsh.

LATR.

F.

lubricipeda

18081

1807 pruni

1808 fenestrella

1758 pellionella

1758 viridana.

1777 apiforme.

1807 io

L.

1758

L. $\quad 1758$

L. $\quad 1758$

L. $\quad 1758$

L. $\quad 1758$

L. $\quad 1758$

F. $\quad 1787$

L. $\quad 1758$

L. $\quad 1758$

I. $\quad 1758$

L. $\quad 1758$

L. $\quad 1758$

L. $\quad 1758$

L. $\quad 1758$

L. $\quad 1758$

L. 1758

L. $\quad 1758$

L. $\quad 1758$

L. $\quad 1758$

L. $\quad 1761$

L. $\quad 1764$

L. $\quad 1758$

L. $\quad 1758$

L. $\quad 1758$

L. $\quad 1758$

L. $\quad 1758$

L. $\quad 1758$

\begin{tabular}{l|l} 
SCOP. & 1763
\end{tabular}

L. 1758

I. $\quad 1758$

\begin{tabular}{l|l} 
Clemer & 1759
\end{tabular}

L. $\quad 1758$

L.

1761

L. $\quad 1767$

L. $\quad 1758$

1804 aesculi .......

1775 Ifilipendulae ...

Lit.: Staudivger \& Rebel, Cat. Lepidopt. paläarkt. Fauna, ed. 3 v. 1.2 1901. 


\section{Coleoptera.}

Acilius ...... Le. LeH 1817 sulcatus Acrocinus.... IL. IL. 1806 longimanus.... Adelocera.... LATr. Adesmia...... Fisch.-

Adimonia ..... IT ALDH.

Adrastus .... Eschz.

Agelastica .... Redr.

Agonum

Agrilus .

Agriotes

Amara

Amorplioce-

phalus ....

Anisoplia

Anobium

Anomith......

Anophthalmus.

Anthaxia .....

Antlia ...

Anthicus

Box.

Sтерн.

Eschz.

Box.

Anthonomus.

Antlurenus

Antluribus

Apate

Aphodins

Aphthona

Apion.

Apoderus

Sснӧхн.

1829 fasciata

1822 longipes

1781 tanaceti

1829 limbatus . .

1858 alni ...

1809 nliginosum.

SERT.

F.

1830

1829

biguttatus

1809

lineatus

Sa Motelelie

Sturix

Eschz.

F. Weber

PaYk.

Gers.

L. GEOFFr. 1762 scrophulariae.

Lacord.

F.

ILL.

Aromia .

Asida

Astynomus

Ateucluns

Athouts . .

Atomaria

Atractocerus .

Attagemis

Attelabus

Balaninus

Baris

Batocera

Bembidium . .

Blaps

Bledius . .

1866 albinus .

1775 terebrans

1798 fossor .

Bostrychus....

Cherr.

Herbst

OL.

SERV.

Latr.

STEPII.

F. Weber

Eschz.

Steph.

Palis.

LATR.

L.

Gerar.

GERM.

CAST.

LATR.

$\mathrm{F}$.

1842 euphorbiae .

1797

1807

1833 conyli .

1804

1839

1801

1829

1830

1801

1807 pellio

$175 \mathrm{~s}$ curculionoides

1817

1824 coerulescens .

1810 r'ubus

1804 velox

1775

1832

mortisagia.

LeAch

L. GEOFFR
1832 taulus....

$\begin{array}{cc}\text { L. } & \left.1758^{1}\right) \\ \text { L. } & \left.1764^{1}\right) \\ \text { L. } & \left.1764^{1}\right) \\ \text { F. } & \left.1775^{1}\right) \\ & \\ \text { L. } & \left.1758^{3}\right) \\ \text { F. } & \left.1787^{1}\right) \\ \text { L. } & \left.1758^{3}\right) \\ \text { ER. } & \left.1837^{1}\right) \\ \text { F. } & 1837^{3} \text { ) } \\ \text { L. } & 1758^{3} \text { ) } \\ \text { PANz. } & \left.1797^{1}\right)\end{array}$

Gerni.

$\left.1817^{1}\right)$

F.

$\left.1775^{1}\right)$

L.

$\left.1761^{2}\right)$

F.

$1775^{1)}$

Sturu

1844 1 )

Felite

$\left.1841^{1}\right)$

F.

$\left.1775^{1}\right)$

Rossi

$1792^{1)}$

L.

17581)

L.

$\left.1758^{1}\right)$

L.

$17581)$

Palli.

L.

$\left.1772^{4}\right)$

SchrdNe

$\left.1761^{1}\right)$

Heribst

I.

$\left.1781^{2}\right)$

L.

Sol.

L.

L.

L.

Sтерн. $1836^{1}$ )

$17971)$

$\left.1758^{1}\right)$

$\left.1758^{1}\right)$

$\left.1836^{1}\right)$

$\left.1758^{3}\right)$

$\left.1758^{\prime}\right)$

$\left.1758^{1}\right)$

L.

17581)

L.

$\left.1761^{1}\right)$

L.

$\left.1758^{1}\right)$

L.

$\left.1758^{1}\right)$

Scop.

L.

$\left.1763^{1}\right)$

L.

$1758^{1)}$

L.

1761$.

Gen.

$\left.1758^{\prime}\right)$

L. 
Brachyuus .... F. WEBer $1801 \mid$ crepitans ....

Brachonyx ....

Brichycerus...

Brachyderes. . .

Bracliytarsus .

Brenthus .....

Brontes

Broscus

Bruchus

Buprestis .....

Byilums

Byturus

Calandra

Callidium

Calosoma

Campylus

Cantliaris

Carabus

Cardiophorus . .

Cassida.......

Cebrio

Cerambyx.....

Cetonia.

Centhorliynchus

Chalcophora...

Chalcosoma ...

Chrysobothrys .

Chrysomela ...

Cicindela

Cis

Cistela

Cleonus

Clerus .

Clytra

Clytus.

Cnemidotus
Chaetociema . .

Chilocorus.....

Cionus

Claviger

SсHӧнн.

OL.

SchönH.

Schonn.

F.

F.

Panz.

L.

L.

L.

LATR.

Clatrv.

$\mathrm{F}$.

F. W Feber

FISCH.-

WALDH.

.L.

L.

Eschz.

L.

OL.

L.

F.

Gerni.

STEPH.

SoL.

Hope

LEACH

Eschz.

L.

L.

Clairy.

LATR.

F.

Preysst.

(1736) vesicatoria . . . .

1758 amratus . .

1829

1758 vignttatus .

1758

1790 gigas

1758

1775

$182 !$

1831

1833

1837

1815

1829

1758 staphylea

1758 campestris

1798

scrophnlariae . .

1796 boleti

1775 ceramboides . . .

1790 testaceus

SсHӧхн.

L. Geoprrr.

LAICH.

IAICH.

ILL.

1826 sulcirostris

1762

1781

1784

1802
Cneorhimus ....

Coccinella.

ScHöNH.

L.

Collyris

F.

F.

1826 geminatus

1758 septem-

punctata.

Colydium

F.

I.

$\left.1761^{1}\right)$

PAYK.

L.

L.

F.

L.

L.

I.

L.

I.

I.

F.

L.

L.

L.

I.

L.

L.

OL.

L.

F.

Scop.

L.

Germ.

Marshaum

L.

L.

L.

F.

L.

L.

L.

Scop.

L.

PremssL.

L.

L.

L.

I.

DUFT.

F.

$1758^{1}$ )

$\left.1761^{1}\right)$

$\left.1790^{1}\right)$

$1758^{1}$ )

$\left.1787^{1}\right)$

$\left.1763^{1}\right)$

$\left.1758^{7}\right)$

$1824^{1}$ )

$1802^{1}$ )

$\left.1758^{1}\right)$

$\left.176 t^{1}\right)$

$\left.1758^{1}\right)$

$1794^{1}$ )

$\left.1758^{1}\right)$

$1758^{1}$ )

$1758^{1}$ )

$17\left(63^{1}\right)$

$\left.1761^{1}\right)$

$1790^{1}$ )

$1758^{2}$ )

$1761^{1}$ )

$\left.1758^{1}\right)$

$1758^{2}$ )

$1805^{2}$ )

$1787^{1}$ )

L.

1758 1)

F.

$1787^{1)}$

F. 
Copris........ L. Geoffr. $1762 \mid$ lunaris

Corymbites....

Corynetes.

Crioceris.

Cryptoceplalus

Cryptophagus

Cryptorhynchus

Crypturgus...

Cucujus

Cupes

Cybister

Cychrus

Cyphon

Dascillus

Dasytes

Dendroctonus. .

Dermestes

Desmonota....

Dicranorhina ..

Dictyopterus

Ditomus

Donacia

Dorcadion

Dorcus ...

Doryphora

Dorytomus

Drasterius

Drilns ...

Dynastes

Dyschirius

Dytiscus.

Elaphrus

Elater.

Eleodes

Emenadia

Endomychus

Epilaclina..

Eroates

Erotylus.

Euchirus

Eumolpus

Falagria

Galeruca

Geotrypes

Gibbiun . .

Gnathocerus. .
LATR.

HERBST

L. GEOFFR.

L. Geofrr.

Herbst

ILL.

ER.

F.

F.

J. Curt.

F.

PAIK.

LATR.

PaYk.

ER.

L.

Hope

Hope

LATR.

Bon.

F.

Dalir.

IV. M'Leay

ILL.

STEPH.

Eschz.

OL.

KIRBY

Box.

L.

F.

L.

Eschz.

Cast.

Panz.

Chifrr.

SERv.

F.

Burry.

F. IV EBER

MANAERH.

L. GEOFFR.

LATR.

Scop.

Thunb.
1834 aeneus

1791 coeruleus

1762 merdigera

1762 coryli

1792

1:07 lapathi

18.36 pusillus.

1775 sanguinolentus.

1801 capitatus .....

1832 roeseli.

1794 rostratus

1798 valiabilis

1796

1798

1836

1758 lardarius

1839 variolosa

18:37 micans

$1+29$ rubens

1809

1775

1817

1819 parallelepipedus

1807 aestuans . . . . . .

1831 tortrix

1829

1790

$18+0$

1813

1758

1801 uliginosus .

1758 .

1829

1840

1795

1844

1832

1775

1840

1801

1830

1762

1796

1777

1814
L.

L.

Degrer

L.

L.

Herbst

L.

GILL.

L.

F.

Fuessur

L.

Thunb.

L.

L.

KLG.

L.

F. WEBER

Drury

GILL.

DEJ.

F.

L.

I.

L.

I.

Rossi

Rossi

L.
DE.J.
L.
F.
L.

Eschz.

GEBL.

L.

F.

L.

L.

L.

F.

Patk.

L.

L.

F.

F. $\left.1758^{1}\right)$

$1758^{1}$ )

$\left.1775^{1}\right)$

$1758^{1)}$

17581)

$\left.1793^{1}\right)$

$1758^{1)}$

$\left.1813^{7}\right)$

$1758^{1}$ )

$\left.1801^{1}\right)$

$\left.1775^{1}\right)$

$\left.1761^{1}\right)$

$\left.1787^{2}\right)$

$\left.1758^{1}\right)$

17611)

$\left.1794^{2}\right)$

$\left.1758^{1}\right)$

$\left.1801^{1}\right)$

$\left.1773^{1}\right)$

$\left.1817^{1}\right)$

$1826^{1}$ )

$\left.1775^{1}\right)$

$\left.1758^{1}\right)$

$17581)$

$17581)$

$1761^{1}$ )

$\left.1790^{2}\right)$

$\left.1790^{1}\right)$

$\left.1758^{1}\right)$

18251)

17581)

18011)

$1758^{1)}$

$18291)$

$\left.18: 30^{1}\right)$

$\left.1758^{1}\right)$

$17751)$

$\left.1761^{1}\right)$

$\left.1758^{1}\right)$

17581)

17751)

$\left.1789^{1}\right)$

$\left.1758^{3}\right)$

$\left.1761^{1}\right)$

17811)

17981) 
Guathosia..... FISCH.-

Gnorimus IVALDH.

Goliathus SERv. Lir.

Gymmetroll.... SсHÖNH.

1821 glabra

Fisch.-

Gyrinus

L. Geoffrr.

1825 nobilis

IV ALDH.

Haliplus LATR.

1801 giganteus

L.

1826 noctis

Haltica.

Harpalus

Heilipus

Helodes

Helops

Hispa

1762

d. GEOFFr.

1806

LATR.

1762

Gerir.

1804

LATR.

F.

L.

L.

Hister

TuL.

Hydrobius

LEACH

1824

natator

1796

1775

1767

1758

1803

LATR.

1817

Hydrochares*).

Hydrophilus ...

Hydroporus

L. Geofrr.

1825

ulvus.

oleracea

1762

Clatrv.

1806

Hylastes......

Er.

BEDEL

I 836

aeneus

catagraphus

minuta

coeruleus

LM.

Herbst

L.

F.

L.

F.

Germ.

L.

L.

L.

Herbst

L.

L.

L.

L.

F.

PAYK.

Hylastinus ....

Hylecoetus....

LATR.

F.

1876

ater

1806 dermestoides

Marshaje

$\left.1821^{1}\right)$

Hylesinus .

Hylobius

Hylurgus

Inca

Ips

1801

GerM.

1817 abietis

LATR.

1807

Serv.

F.

1825

ligniperda....

1776

clathratus

adripustu-

Julodis

Eschz.

1829

latus.

Lacon

Cast.

F.

F.

Lamia

Cast.

1836

1775

1775

Laemophloeus

1840

JAQUEL.

1859

Lampyris . .

Larinus

Lathridius

Lathrobium

Lebia

Leistotrophus

Lema

Leptinotarsa

Leptoderus. .

Leptura

Lepyrus

L. GEOFFr. 1762

GeriI.

1824

Herbst

1793

Grav.

1806

LATR.

Perty

1804

F.

STÅL

SturM

L.

I.

F.

17581)

$\left.1817^{1}\right)$

$\left.1795^{1}\right)$

$\left.1761^{1}\right)$

180] ${ }^{1}$ )

17581)

$\left.1775^{1}\right)$

$\left.1824^{1}\right)$

$1758^{2}$ )

17641)

17671)

$\left.1792^{1}\right)$

17581)

$\left.1761^{1}\right)$

17611)

$\left.1761^{1}\right)$

$\left.1792^{1}\right)$

$\left.1800^{7}\right)$

$\left.1802^{7}\right)$

$1761^{2}$ )

L.

$\left.1787^{7}\right)$

F.

$\left.1785^{1}\right)$

Ox.

$\left.1792^{7}\right)$

$\left.1792^{1}\right)$

L.

F.

L.

17611)

17871)

L.

17581)

L.

17581)

StEPH.

L.

$1758^{1}$

L.

$1831^{1}$ )

$1758^{2}$ )

$1758^{2}$ )

F.

$\left.1775^{1}\right)$

Degeer

$\left.1775^{2}\right)$

F.

$1792^{2}$ )

L.

$1761^{2}$ )

F.

$1792^{3}$ )

L.

$\left.1758^{1}\right)$

SAY

18231)

Schm.

$\left.1832^{7}\right)$

L.

$1758^{3}$ )

SchöNH.

1758 virens

L.

$1771^{3}$ ) 
Lethrus

Limobius

Limonius

Lina

Lixus

Longitarsus ...

Lucanus .

Luciola .

Lycoperdina...

Lymexylon .

Macrochirus...

Macrotoma....

Magdalis

Malachins.....

Malthinus.....

Malthodes

Manticora

Necinus .

Megasoma

Meligethes

Meloe

Melolontha....

Mesosa

Metoecus

Miarus

Microzoum

Molytes

Mononychus.

Nordella.

Mormolyce ....

Myelophilus

Mylabris .

Myrmedonia. .

Nanophyes ....

Nebria

Necrophorus

Necydalis .

Nemosoma

Niptus

Nitidula

Nosodendron .

Notoxus

Oberea

Oedemera

Omalium

Omophron

Scop.

ScHöNH.

Eschz.

REDT.

F.

LATR.

Scop.

CASt.

LATR.

F.

SсHÖNH.

SERv.

GerM.

F.

LATR.

Kiesentr.

F.

Germ.

KIRBY

Steph.

L.

F.

SERv.

Gerst.

Steph.

REDT.

SchÖNH.

Germ.

L.

Hagenb.

ЕІонн.

F.

ER.

Schönh.

Latr.

F.

I.

LAtr.

BoIELD.

F.

LATR.

L. GEOFFr

Muls.

OL.

Grav.

LATR.
1777 cephalotes

1817

1829 cylindricus

1858

1801

1829 atricillus

1763 cervus.

1833 italica

1829 bovistae

1775 navale

1838 longipes

1832 palmata

1818 cerasi

1775 bipustulatus . .

1806 fasciatus

1852

1801

1821

1825

1830 aeneus

1758 proscarabaeus

1775 vulgaris

1835 nebulosa.

1855 paradoxus

1831 campanulae . .

$18+9$ tibiale

1826 germanus

1824 psendacori

1758 aculeata

1825

1879

1775 cichorii .

1837 canaliculata ...

1845 lythri

1806 brevicollis

1775

1758

1804 elongatum

1856 hololeucus

1775

1807

1762

1839

1795

bipustulata.

fasciculale.

monoceros

1802 rivulare

1804 limbatus
PALL.

Herbst

$\left.1771^{3}\right)$

PAYK.

L.

$1795^{3}$ )

Herbst

L.

L.

L.

F.

L.

Drury

F.

L.

L.

Ol.

KIESENT.

F.

Germ.

F.

F.

L.

F.

F.

$1771^{3}$ )

$1758^{3}$ )

$1784^{2}$ )

$1761^{2}$ )

17581)

$\left.1758^{2}\right)$

$\left.1792^{2}\right)$

$\left.1761^{2}\right)$

17931)

1801 ${ }^{1}$ )

$\left.1758^{2}\right)$

$1761^{2}$ )

$1790^{2}$ )

$\left.1852^{2}\right)$

$\left.1801^{1}\right)$

$1821^{3}$ )

$\left.1775^{1}\right)$

$\left.1775^{3}\right)$

$1758^{3}$ )

$1775^{3}$ )

L.

$1781^{3}$ )

$1761^{3}$ )

I.

$\left.1758^{2}\right)$

F.

$1781^{2}$ )

L.

$1758^{3}$ )

F.

$1801^{3}$ )

L.

$\left.1761^{1}\right)$

HAGENB.

$1825^{1}$ )

L.

$1758^{8}$ )

L.

$\left.1758^{1}\right)$

F.

17871)

F.

1787 1 )

F.

$1792^{1}$ )

L.

1761 $\left.{ }^{1}\right)$

L.

17581)

I.

$\left.1761^{1}\right)$

FALd.

L.

$1835^{2}$ )

OL.

$1761^{1}$ )

$\left.1790^{1}\right)$

L.

$1761^{1}$ )

L.

17581)

L.

17671)

PAYK.

$\left.1789^{1}\right)$

F. 


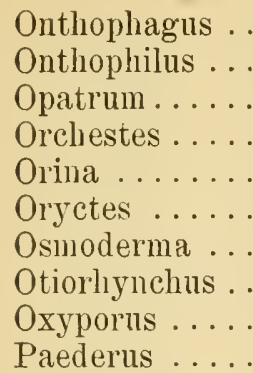

Panagaells

Parnus

Paussits

Pedinns

Pelobius

Peltis

Philonthus

Phosphaenus

Phyllobius

Phyllopertha..

Phyllotreta ...

Phytonomus ...

Pimelia.

Pissodes

Platycerus

Platysoma

Poecilonota

Polydrosus

Polyphylla

Prionus

Pristonychus .

Pselaplius

Psylliodes

Pterostichus...

Ptinus

Pyrochroa

Pyrophorus

Pytho

Rhagium

Rhipidius .....

Rhizophagus .

Rhizotrogus

Rhynchites.

Rhynchophorus

Rhyncolus ....

Rhysodes

LATR.
LEACH
F.
ILL.

LACORD.

ILL.

Serr.

Germ.

F.

F.

LATR.

F.

I.

LATR.

ScHÖNH.

ILI.

J. Cunt.

CAST.

Geric.

Steph.

Foudras

SCHONH.

$$
\text { F. }
$$

Geru.

L. Geofrer.

LEACH

Eschz.

Geru.

IV. HARR.

L. GEOFFr.

DE.J.

Herbst

Latr.

Bow.

L.

L. GEOFFH

ILI.

LATR.

F.

Thunb.

Herbst

LATR.

Herbst

Herist

Geril.

DALite.
|1807 |camelus

1817 striatus .

1775 sabulosum

1804 quercus .

1874 gloriosa

1798

1825 eremita.

1824 ligustici

1792

1775

1804

1792

1775

1796 femoralis

1808 hermanni

1798

1825 splendens

1833 hemipterus

1824 argentatus

1830 horticola.

1860 nemorum

1826 punctatus

1775 bipunctata

1824 notatis .

1762

1817

1829

1824

1842

1762

1828

caraboides

depressum

rutilans

undatus

fullo

1792

1829

1809

1767

coriarius

oblongus.

heisei

chrysocephalus

1762

1809

1796

1775 inquisitor

1806 pectinicornis

1793

1825 stolstitiali

1797

1795

1824 truncorum

1823 sulcatus
F.

F.

L.

I.

F.

I.

L.

I.

L.

L.

L.

ER.

FrIV.

L.

F.

I.

F.

L. Geoffr.

L.

L.

L.

F.

F.

F.

L.

F.

F.

F.

L.

L.

Ders.

HERBST

L.

SChallier

L.

L.

L.

L.

L.

Thuns.

F.

L.

L.

L.

GeriI.

F.
17871)

18011)

$1761^{1}$ )

$1758^{1)}$

$\left.1781^{1}\right)$

17581)

17581)

$\left.1758^{1}\right)$

$\left.1761^{1}\right)$

$\left.1761^{1}\right)$

$\left.1758^{1}\right)$

$\left.1848^{1}\right)$

$\left.1835^{1}\right)$

17671)

1792 1 )

$\left.1761^{1}\right)$

17921)

$1762^{1)}$

17581)

$1761^{1}$ )

$1758^{1)}$

$\left.1775^{1}\right)$

$\left.1781^{1}\right)$

$\left.1787^{1}\right)$

$\left.1758^{1}\right)$

$17871)$

17771)

$\left.1781^{1}\right)$

17611)

17581)

$1828^{1}$ )

$\left.1792^{1}\right)$

17581)

$\left.1783^{1}\right)$

17611)

1761 1 )

$\left.1764^{1}\right)$

17671)

$\left.1758^{3}\right)$

$1806^{1}$ )

$\left.1801^{1}\right)$

$\left.1761^{1}\right)$

17581)

$\left.1764^{1}\right)$

$\left.1824^{1}\right)$

17871) 
Rosalia ........
Sagra .......

Salpingus

Saperda

Saprinus......

Scarites

Scolytus

Scydmaenus ...

Serica .

Sibinia

Silpha.

Silvanus ......

Sisyphtıs .

Sitaris

Sitona.

Smicronyx ....

Sphodi'us .....

Staphylinus ...

Stenus

Strangalia

Strophosomus .

Telephorus....

Temnochila ...

Tenebrio

Thamnurgus . .

Tomicus

Trachys .....

Trechus

Tribolium .....

Trichius

Trichodes.....

Trichopteryx . .

Trogosita.....

Trox.........

Tychius

Valgus

Xyleborus

Xylophilus

Zabrus .
SERv. $1833 \mid$ alpina.

F.

GILL.

F.

Er.

F.

L. Geoffr.

Latr.

IV. M'Leay

Germ.

L.

Latr.

LATr.

LATR.

GerMI.

SсHÖNH.

Clatiry.

L.

LATr.

Serv.

SCHÖNH.

SchäfFer

WESTW.

L.

ЕІснн.

LATR.

F.

Clairt.

W. M'Leay

F.

Herbst

KIRBY

OL.

F.

SснӧNн.

Scriba

Етснн.
1792 jurpurea

1810 ater

1775 carcharias

1834 aeneus

1801 arenarius

1762

1802 tarsatus . .

1819 brunnea

1824 femoralis

1758

1807 .

1807

1802 muralis .

1824

1843 jungermanniae.

1806 leucophithalmus

1767 erytliropterus. .

1796 biguttatus ....

1835 ar

1826 coryli

1766 fuscus .

1835 coerulea

1758 molitor .

1864 kaltenbachi

1810 typographus.

1801 1

1806

ninuta ......

minutus

1825 ferrugineum . .

1792 fasciatus. . . . .

1792 apiarius

1826 fascicularis.

1790 mauritanica

1792

1792 sabulosus ....

1826 quinque-

maculatus. .

1790 hemipterus .

1864 monographis . .
Latr.

Clairv.

1825

1806

lygmaeus.....

Lit.: 1) Lacordaire, Gen. Col., v. $1-121854-1876$.

2) Seidlitz, Fauna Balt., od. a 1887-i891.

3) Redtendacher, Fauna Austr., ed 31874.

4) Legne in: Ann. Soc. ent. France, 1909 p. 480.

5) Lesne in: Ann. Soc. ent. France, 1898 p 442.

-) Castelinu \& Gory, Mlonogr. Buprest.

7) Reitter, Bestimmungstabellen.

8) Eicnнoff, Europ, Borkenkäf. \begin{tabular}{c|c} 
L. & $\left.1758^{1}\right)$ \\
A. Lсhт. & $\left.1796^{1}\right)$ \\
PAYK. & $\left.1798^{1}\right)$ \\
L. & $\left.1758^{1}\right)$ \\
F. & $\left.1775^{1}\right)$ \\
Bon. & $\left.1813^{1}\right)$ \\
RATZEB. & $\left.1837^{1}\right)$
\end{tabular}

Рн. W. MÜLL. $1822^{1}$ )

L.
GERM.
L.
L.
L.

Fonst.

$\mathrm{L}$.

REICH.

L.

L.

L.

Herbst

F.

L.

OL.

L.

$\mathrm{BACH}$

L.

L.

F.

F.

L.

$\mathrm{L}$.

$\left.1758^{1}\right)$

$\left.1824^{1}\right)$

17581)

17581)

17581)

$\left.1771^{1}\right)$

$\left.1758^{2}\right)$

17971)

17611)

17581)

1761 $\left.{ }^{1}\right)$

$\left.1784^{1}\right)$

$\left.1775^{1}\right)$

$\left.1758^{1}\right)$

$\left.1790^{1}\right)$

17581)

$\left.1849^{7}\right)$

17581)

1758 1 )

18011)

1781')

17581)

Herbst

17581)

L.

17931)

L.

17581)

17581)

L.

17581)

L.

F.

17581)

Degeer

$\left.1792^{1}\right)$

F.

17741)

1794 1 ) 
Anhang: Rutelinae, Subfam. der Scarabaeidae.

\begin{tabular}{|c|c|c|c|}
\hline Adoretus & LAP. & 1840 & lobscurus. \\
\hline Anomala...... & SaMOUELIE & 1819 & frisclit. . \\
\hline Anoplognathus & IV. MI'LEAY & 1819 & viridiaenens \\
\hline intichira .... & Escuz. & 1818 & virens.... \\
\hline rachystel'uus . & GUER. & 1830 & prasinus \\
\hline uchiruts .... & BURM. & 1840 & longimanus. \\
\hline eniates...... & IV. KIRBI & 1818 & barbatus. \\
\hline acraspis.... & II. M'Lear & 1819 & cincta ... \\
\hline Parastasia ... & W EsTW. & 1842 & canaliculata \\
\hline Pelidnota .... & II. M'LEAY & 1819 & punctata... \\
\hline haenomeris . & Hope & 835 & ckei.. \\
\hline Platycoelia .. & BURM. & 1844 & valida .... \\
\hline otis. . . . & BuRM. & 1844 & victorina \\
\hline $\mathrm{Pon}$ & SERV. & & bipmetata . . \\
\hline & LATR. & & lineola ..... \\
\hline Spodochlamys & Butra. & & caesarea. \\
\hline
\end{tabular}

F. 1781

F. $\quad 1775$

IV. M'Leat 1819

DRURY 1773

Guér. $\quad 1830$

L. $\quad 1758$

IV. KIRBY 1818

Drery 1782

WESTW. $18+2$

L. $\quad 1758$

Mannerh. 1838

BCRM. 1844

Hope 1840

F. $\quad 1787$

L. $\quad 1758$

BurM. 1855

OHaus (Berlin-Steglitz).

\section{Hymenoptera.}

Agriotypus.... Alysia.

Ammopliila ...

Ampulex......

J. Cunt.

18.2. armatus

J. Corr.

LATR.

IV. KIRBI

1802 manducator

JUR.

1798

(1iv

Paxz.

Scop.

JUR.

1832

1799

1763

1807

Andrena ......

Anthidium ....

Anthophora...

Aphidius......

A pis

Astata

F.

F.

1775 majginata

F.

L.

L.

LATR.

1804 manicatum

NeEs

L.

LATR.

Athalia.

LEACH

Atta

F.

JUR.

F.

Bembex

1802 acervorum

Bethylıs

1818

1758

picipes

1796

mellifica

1817 rosae

1801 cephalotes ....

1807 striatus .

LATR.

Blastophaga...

Bombus ......

Bracon

Gravenh.

LAtr.

F.

Camponotus

1775 rostrata

NeEs

L.

SCHRANK

I.

L.

Cephus

Ceraphron

Cerceris

Ceropales

MAYR

LATR.

JUR.

1802 cenopterus ....

1826 psenes.

LATR.

1802 terrestris

1804 urinator

Chalcis

LATR.

1861

1802

1807

ligniperda.... .

pygmaens .....

sulcatus

1802 arenaria

F.

1796

maculatus.

1789

LEP.

1841 muraria

$\left.1776^{1}\right)$

$\left.1758^{2}\right)$

$1758^{3}$ )

1818

$\left.1761^{4}\right)$

1781

1758

1758

J $\mathrm{CR}$.

L.

Panz.

L.

I.

F.

LATR.

L.

JUR.

L.

F.

L.

1807

1758

Chalicodoma

RETZ.
1801

1758

$1758^{3}$ )

1798

$1802^{10}$ )

1767

1807

1758

1775

1767

$1783^{5}$ ) 


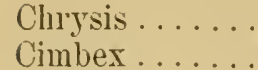

Colletes

Crabro

Crematogaster*)

Cryptus

Cynips

Dasypoda....

Diapria

Dorylus

Dryinus

Dryoplianta ..

Emplyytus.....

Encyltus

Ephialtes

Eucel'il

Englossa

Eumenes

Eurytoma.

Erania.

Formica.

Gorytes

Halictus

Hylotoma .....

Ichneumon

Larra

Lasins

Leptothorax . .

Lophyrus .... .

Lyda.

Masa:is

Megachile.

Megalyra

Melecta

Melipona

Mellinus

Methoca

Microgaster ...

Mintilla

Mymar

Myrmica.....

Nomada

Nisson.

Odynerns
L.

Or.

LATR.

F.

LUND

F.

L.

LATR.

LATR.

F.

LATR.

A. FöRST.

KLUG

LATR.

Schraxk

Scop.

LATR.

LATR.

ILL.

F.

L.

LATR.

LATR.

LATR.

L.

F.

F.

MATR

LATR.

F.

F.

LATR.

WESTW.

LATR.

ILL.

F.

LATR.

LATR.

L.

J. Curnt.

LATR.

Scop.

LATR.

LATR.
1761 ignita

1790 femorata

1802 succinctus

1775) fossorius

1831 sordidula

1804 sponsol .

1758 calicis .

1802

1796 rerticillata

1793 helrolus

180.) formicarius

1869 folii.

1813 cinctus

1809 iufidus

1802 manifestator.

1770 longicornis

1802 cordata.

1802

1807 abrotani

1775 a pnendigaster

1758 l'ufia

1805 mystaceus

180 ร

1802

1758

1793

1804

1855

1802

1804

1793

1802

1832

1802

1806

1790

180 .5

1802 globata .......

$175 \mathrm{~s}$

1832

1805

1770

1796 maculatus....

1802 parietum
I.

1758

L.

1758

L. $\quad 1758^{3}$ )

L. $\quad 1758$

NiL.

F.

$\left.18+9^{10}\right)$

1793

Gir.

1790

F.

$\left.1793^{3}\right)$

LATR. 180:

L.

LATR.

$17\left(j t^{10}\right)$

L.

L.

1805

1758

1758

RossI

L.

17901

1758

L.

$1758^{6}$ )

L.

L.

$\left.1758^{3}\right)$

1758

ILL.

L.

1807

L.

1758

L.

$\left.1758^{10}\right)$

L.

1761

L.

$1758^{3}$ )

I.

1758

1758

RossI

1790

I.

$1758^{10}$ )

F.

$1775^{10}$ )

L. $\quad 1758$

L. $\quad 1758$

F.

L.

1793

$\left.1758^{3}\right)$

TVEST.

1832

Panz.

F.

L.

$\left.1799^{3}\right)$

$\left.1798^{7}\right)$

LATR.

1758

L.

L.

1805

1758

1758

J. Curt.

1832

NYL.

$\left.18+6^{10}\right)$

F.

L. $\quad 1758$ 
Ophion

Osmia.

Oxybelus

Pelopoeus .

Pezomachus

Pheidole.

Philanthus

Pimpla .......

Polistes

Polyergus

Pompilus

Ponera

Proctotrupes

Prosopis .

Pteromalus

Rhodites. .

Rhyssa

Sapygit .

Scelio

Scolia

Sirex

Specodes

Sphex

Steplianus

Stizus........

Telenomns

Tenthredo ....

Tetramorium ..

Thynmus.....

Tiphia

Trachusa .....

Trigona ......

Trigonalys ....

Tryphon ......

Trypoxylon

Vespa

Xiphydria

Syela

Xylocopa
F.

PANz.

LATR.

LATR.

Gravenh.

WTESTW.

F.

F.

LATR.

LATR.

F.

LATR.

LATR.

F.

Swederts

Hartic

Gravenh.

LATR.

LATR.

F.

L.

LATR.

I.

Jur.

LATR.

HALID.

L.

NAYR

F.

F.

JUR.

JUR.

WESTW.

FALL.

LATR.

L.

LATR.

DALM.

LATR.
1798 luteus

1806

1796

1802

1829

$18 \pm 1$ megaceplala

1790 triangulum .

1804 instigator .

1802 biglumis

1805 rufesceus

1798 viaticus

1805 contracta

1796 1

1801 bipunctata

1795 puparum.

1810 rosae.

1829

1796 quinquepunctata

1805 Ingulosus . . . . .

1775 flavifrons

1761 gigas

1805 gibbus

1758 indicus

1807

1802

1833

1758 atra

1855 caespitum.

1775 dentatus.

1775 femorata

1807 ser.

1807 :

1835

1813

1796

1758 vulgaris

1802 camelus

1819 pusilla

1802 violacea

\begin{tabular}{c|l} 
L. & 1758 \\
PANZ. & $\left.1798^{8}\right)$ \\
L. & 1758 \\
LATR. & 1802 \\
L. & 1758 \\
F. & 179311 \\
F. & 1775 \\
F. & 1804 \\
L. & 1758
\end{tabular}

LATR.

L.

LATR.

Paxz.

F.

I.

L.

L.

$\mathrm{F}$.

LATR.

F.

L.

L.

L.

F.

F.

HALID.

L.

L.

F.

F.

Panz.

JUR.

SPIN.

I.

L.

I.

L.

DALAI.

L.
179810)

1758

$1802^{10}$ )

1805

$1804^{2}$ )

1758

1758

1758

1781

1805

1775

1758

$\left.1758^{3}\right)$

1758

1798

1775

1833

1758

175810)

1775

1775

$1805^{9}$ )

$1807^{9}$ )

$18+0$

1761

1758

1758

1758

1819

$1758^{3}$ )

Lit.: Dalla Torre, Cat. Hym., v. 1-10 1892-1902.

1) J. C. Fabricios, Syst. Ent., 1775.

2) J. C. Fabricius, Syst. Piez., 1804.

3) Latreille, Hist. Crust. Ins., v. 1-14 1802-1805.

4) Linne, Syst. Nat., ed. 101758.

5) Lepelfier, Ins Hym., v. 1-4 1836-1846.

6) Scopoli, Annus hist.-nat., 1-5 1769-1772.

7) Illiger, Mag. Isektenk., 1806. 
Lit.: 8) PAxzer, Krit. Revis., 1805, 180 t.

9) Jurine, Nouv. Méth. Hym., 1807.

10) MaYr, Europ. Formic., $1 \subset 61$.

11) Andre, Spec. Hym. II.

Friese (Schwerin) Apidae, Stitz (Berlin) Formicidae, Hasdulirsch (Wien) Rest.

\section{Molluscoiden.}

Bryozoa.

Aetea $\ldots . . . . . .$.
Alcyonidium .

Amathia ......

Bicellaria .....

Bugula

Caberea

Cellepora.....

Cellularia

Crisia .

Cristatella

Flustra .

Gemellaria.....

Hornera

Idmonea .

Lepralia

Lophopus.....

Loxosoma.

Nembranipora .

IIenipea ...... Lux.

Myriozoum. ... (Dox.) PaLL.

Paludicella.... GERT.

Pedicellina.... S SARS

Plumatella.... Lar.

Retepora..... Lx.

Schizoporella..

Scrupocellaria .

Tubulipora....

Vialkeria.......

Vesicularia.
Lux.

Lax.

Lix.

BLAIXT.

OKEN

O. FABR.

Pall.

LaTX.

Cer.

SAY.

Lax.

Lix.

Jонхет.

Dumort.

KEF.

Hoiss.

BENED.

Lis.

FIEN.
1812 anguina

1813 gelatinosum

1812 lendigera .

1830

1815

1816 elis

1780 I

$1766 \mathrm{P}$

1812 e

1798

1767

1811 loricata

1821

1821

1838
1835

1862

1834

1816

crystallinus ...

singulare .....

pilosa ........

te

(1750)

$1766^{\circ}$

1838 ehrenbergii ...

1835 cernua

1816 repens

1801 cellulosa

1880 hyalina.

$18+4$ sermposa

1816 flabellaris

$182 ; 3$ ura

ura.........

spinosa.

Lit.: 1) Hartueyer in: Brauer, Süßw., v. 191909.

2) Hincks, Brit. Mar. Polyzoa, v. 1, 1880.

\section{Brachiopoda.}

Crania ...... Retz.

Discina...... Lx.
1781 anomala

1819 striata
L.

L.

L.

L.

L.

FLeMT.

L.

Busk

L.

Cur.

L.

L.

Lix.

L.

ELL. \&

SOLAND.

PALI.

KEF.

I.

ELL. \&

Solaxd.

PALL.

1766

BENED.

PAll.

L.

L.

L.

L.

$\left.18+8^{1}\right)$

$\left.1771^{2}\right)$

17.581)

$\left.1758^{2}\right)$

$17\left(i 7^{2}\right)$

O. FABR.

L.

L.

$\left.1767^{2}\right)$

$\left.1780^{2}\right)$

$\left.1758^{2}\right)$

$1758^{2}$ ) 


\begin{tabular}{|c|c|c|c|c|}
\hline Lingula ...... & BRUG. & 1791 & anatina..... & Liг. \\
\hline Terebratula ... & MÜLL. & 1776 & vitrea ... & Born \\
\hline Terebratulina . & Orв. & 1847 & $\begin{array}{l}\text { caput } \\
\text { serpentis }\end{array}$ & \\
\hline
\end{tabular}

Lit.: Oenlert in: P. Fischer, Man. Concyl., fase. 111887.

1st $=$ Agrees with Simpen 1900 P. H. 8.h. M. V.22
Mollusca.

Lamellibranchiata.

\begin{tabular}{|c|c|c|c|c|}
\hline $\begin{array}{l}\text { Anatina ........ } \\
\text { Anodonta. }\end{array}$ & $\begin{array}{l}1818 \\
1799\end{array}$ & $\begin{array}{l}\text { anatina ........ } \\
\text { cyonea. }\end{array}$ & L. & $\begin{array}{l}1758 \\
1758\end{array}$ \\
\hline Arca........ & 1758 & noite ........ & L. & $\frac{10}{1758}$ \\
\hline Astarte....... & 1816 & sulcata ...... & Da Costa & 1778 \\
\hline Avicula ...... & 1789 & hil'undo . . . . . . & L. & 1758 \\
\hline Cardita....... & 1789 & calyculata .... & L. & 1758 \\
\hline Cardium ....... & 1758 & eostattrm aculeater. & L. & 17.58 \\
\hline Chama ........ & 1758 & lazarus . . . . . . & L. & 1758 \\
\hline Corbula ...... & 1797 & sulcata...... & Brug. & 1818 \\
\hline Crassatella.... & 1799 & kingicola ..... & Lм. & 1818 \\
\hline Cuspidaria ..... & 1840 & cuspidata ..... & OL. & 1792 \\
\hline Cyprina ....... & 1818 & islandica...... & I. & 1767 \\
\hline Dollax ........ & 1758 & Aenticulata tausio & wis L. & 1758 \\
\hline Dreisseua ...... & $18: 35$ & polymorpha ... & PALL. & 1771 \\
\hline Gastiochaena.. SPrEngL. & $178: 3$ & hians........ & Chenin. & 1788 \\
\hline Glycimeris ... to to & 1799 & glycimeris .... & BURAL. & 177858 \\
\hline Isocardia ..... & 1799 & corduta...... & L. & 1758 \\
\hline Leda.......... & 1817 & pella ......... & L. & 1758 \\
\hline Lima. . . . . . . . & 1784 & scabra $\ldots . .$. & Born & 1778 \\
\hline Lucina ...... . & 1792 & pensylvanica .. & L. & 1758 \\
\hline Lyousia ....... & 1822 & norvegica ..... & Сhemin. & 1788 \\
\hline Nactra ........ & 1767 & Stult tavum & $I$ & 1758 \\
\hline Malleus........ & 1799 & malleus... & $\mathrm{L}$ & 1758 \\
\hline IIargaritana.... & 1817 & margaritifera.. & L. & 1758 \\
\hline Meleagrina.... & 1819 & margaritifera.. & L. & 1758 \\
\hline Modiola ....... & 1801 & modiolus.... & L. & 1758 \\
\hline Montacuta.... & 1819 & substriata.... . & МоNт. & 1808 \\
\hline Mra ........ & 1758 & truncata ...... & $\mathrm{L}$. & 1758 \\
\hline Mytilus....... & 1758 & edulis. & $\mathrm{L}$. & 1758 \\
\hline Nucula ... . . . . & 1799 & nucleus & L. & 1758 \\
\hline Ostrea ....... & 1758 & Lerlulis. & I. & 17.58 \\
\hline Pandora ....... & 1792 & inaequivalvis & L. & 1758 \\
\hline Pecten ....... Wucur In.1776 & 1799 & jacobaetts mast: & L. & 1758 \\
\hline Pectunculus ... L Lar. & 1799 & glycymeris .... & $\mathrm{L}$. & $1758=$ \\
\hline Petricola ..... & 1801 & lamellosa .... & LaI. & 1818 \\
\hline Pholas ....... & 1758 & dactylus ...... & L. & 1758 \\
\hline
\end{tabular}


C. APstein.

\begin{tabular}{|c|c|c|c|c|c|}
\hline Pinna & L. & 1758 & |robilis ?ud!? & L. & 1758 \\
\hline Psammobia. & Lir. & 1818 & |jespertina .. & СHEMN. & 1782 \\
\hline Saxicava .. & FleUR. & 1802 & I'llgosa ... & & 1758 \\
\hline obicularia & Schum. & 1817 & piperata blava 9 & Costa Gur. & 1791 \\
\hline Solen & L. & 1758 & vagina...$\ldots$ & L. & 1758 \\
\hline Sphaerium ... & Scop. & 1777 & corneum .. & $\mathrm{L}$. & \\
\hline Spondylus. . & L. & 1758 & gaederopus & L. & \\
\hline & MEG. & 1811 & pullastra lit mí & HOXт: $\mathcal{L}$ & 1808 \\
\hline & L. & 1758 & virgata ....... & L. & 1758 \\
\hline Teredo & L. & 1758 & navalis .... & L. & 178 \\
\hline Tridacna ... & BRUG. & 1789 & gigas.. & L. & 1758 \\
\hline Trigonia . . . . . . & BRUG. & 1789 & margaritacea . & Lis. & $180 t$ \\
\hline & RETZ. & 1788 & pictor & L. & \\
\hline nus . . . . . . & L. & & mercenaria.. & L. & 78 \\
\hline
\end{tabular}

Scaphopoda.

Dentalím.... L L. $|1758|$ elephantinum... L. L. 1758 op.94

Placophora.

Chiton ...... L. $\quad 1758 \mid$ tuberculatus...| L. 1758

Gastropocla.

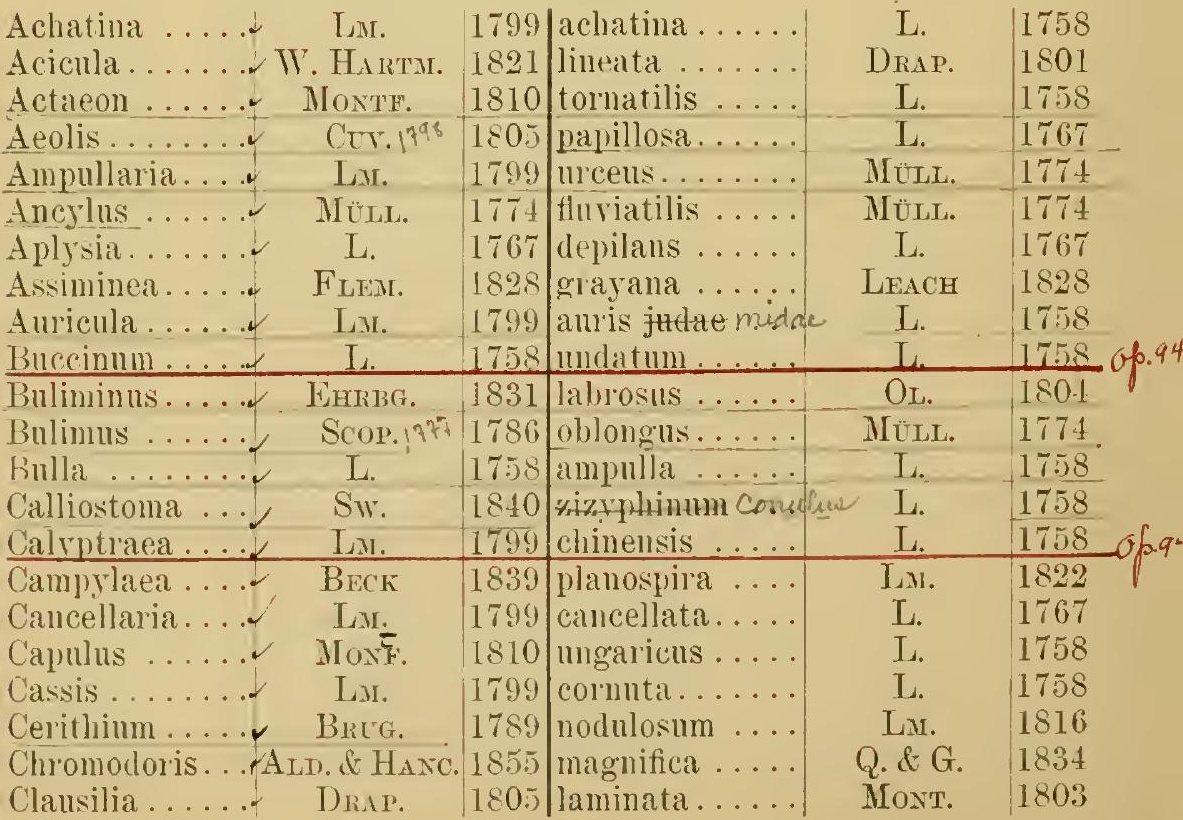


Columbella.... L L

Conus

Crepidula

L.

1799 mercatoria

Cyclostoma ... Drap.

Cypraea ...... L.

Delphinula

Lis.

Dolimm ...... (Нuмгн.)

Doris

L.

Eulima .......

Risso

Fasciolaria....

Fusts ....... Lu.

Haliotis ...... L.

Helicina ...... Lur.

Helix

Lis.

Hydrobia ..... TV. HART'r.

Lamellaria..... Mont.

Limax ....... L L.

Littorina ..... FE FE

Lymmaea.

LiI. 179

Mlargarita

LEACH

Lis.

Marginella .....

Melania

Lis.

Mitra

Lis.

Iurex .......

I.

Narica

ORB.

Nassa

Lis.

Natica

SCOP.

Nerita

L.

RAF.Sam

Odostomia

Fleit.

Oliva

Brutr.

Ovula ........ Brovtr.

$\uparrow$ Paludina .....

Persona .......

Lin.

MoNTF.

Phasianella

LiI.

Pliysa

Planorbis

DRAP.

Plemrotoma

Pleurophyllidia/

MÜLL.

LiI.

МЕeк.

Pomatias

STLD.

Proserpina .... Sow. II.

Pterotrachea. Fonsk.

Pterocera.....

Pupa.

LiI.

Drap.

Pripura ...... Brog.

Pyramidella... Lu.

1758 marmoreus ... .

1799 porcellana

1801 elegans.

1758

1803 laciniata ......

1797 galea

1758

1826 polita .......

1799 tulipa

1799 colus

1758 tuberculata

1799 neritella

1758 pomatia

1821 stagnalis

1815 perspicua

1758 maximus.

1821 littorea.

1804 stagnalis

1819 helicina

1799 glabella

1799 amalula

1799 episcopalis

17.58

$18+1$ cancellata

1799 mutabilis

1777 canrena

1758

1815

1813

1789

1789

1816

1810

1804

1801 fontinalis

1771 coruens.

1799

1816 undulata.....

1789

1839

1775

1799

1805

1789

1799 dolabrata
I.

1. 1758

L. $\quad 1758$

MÜLL. $177 \pm$

L. 1758

Lir. $\quad 1819$

L. $\quad 1758$

L. $\quad 1758$

L. $\quad 1758$

L. $\quad 1758$

L. $\quad 1758$

L. $\quad 1758$

LiN. $\quad 1822$

L.

L.

L.

1758 op.94

1767: hace to

1758

L.

175806.94

1758

1758

L.

O. FABR.

1780

L.

1758

L.

L.

$1758=$ Fina?

L.

1758

1758

Chemr.

1788

L.

L.

1758-Cutru

1758

1758

L.

Irif. 7 m. 1822

MonT.

L.

1808

Li:

1758

L. $\quad 1758$

L.

L.

$1758=$ Viripas

Gis.

L.

1758

1791

1758

1758

L.

MECK.

1758 Jursis

Drap.

1816

Gray

1805

Forsk.

1842

L.

1775

L.

1758

L.

1758

L. 


\begin{tabular}{|c|c|c|c|c|c|}
\hline Ranella........ & Lir. & 1816 & bufonia . ....... & Gus. & 1788 \\
\hline Rissoa........ & FREM. & 1814 & ventricosa .... & DESM. & 1814 \\
\hline Rostellaria.... v & LII. & 1799 & fusus $\ldots \ldots \ldots$ & L. & 1767 \\
\hline Scalaria ...... & LiI. & 1801 & scalaris....... & L. & 1758 \\
\hline Solarium ..... & Lu. & 1799 & perspectivum . . & L. & 1758 \\
\hline Strombus . & I. & 1758 & giguts \& : ?: . . . & L. & 1758 \\
\hline Succinea....... & Drar. & 1801 & putris ......... & $\mathrm{L}_{\mathrm{n}}$ & 17.58 \\
\hline Telebra ....... & BRUG. & 1789 & subulata ...... & L. & 1767 \\
\hline Tetliys ....... & L. & 1758 & leporina ...... & L. & 1758 \\
\hline Trochus ....... & L. & 1758 & niloticus . . . . . & L. & 1767 \\
\hline Truncatella ... & Risso & 1826 & trumcatula ... & Drap. & $180 \bar{J}$ \\
\hline Turbinella .... . & Lir. & 1799 & pyitum $\ldots \ldots$ & L. & 1758 \\
\hline Turbo ....... & L. & 1758 & marmoratus ... & I. & 1758 \\
\hline Turritella..... & LiI. & 1799 & ter"ebra . . . . . . . & L. & 1758 \\
\hline Umbonium . . . . & LINK & 1807 & restiarium .... & L. & 1758 \\
\hline Umbrella ..... & Lir. & 1819 & sinica ....... & G.I. & 1788 \\
\hline Taginula ..... & FER. & 1821 & alta.......... & FÉR. & 1821 \\
\hline Vermetus ..... & BRUG. & 1789 & adansonii ..... & DAUD. & 1800 \\
\hline Vitrina ........ & DrAP. & 1801 & pellucida ..... & MÜLL. & 1774 \\
\hline Voluta ...... & L. & 1758 & vespertilio .... & $\mathrm{L}$ & 1758 \\
\hline Senophora.... & ISCH.- & 1807 & trochiformis... & BORN & 1778 \\
\hline
\end{tabular}

Lit.: P. Fischer, Man. Conchyl., 1880-1887.

Thiele (Berlin).

Pteropoda.

\begin{tabular}{|c|c|c|c|}
\hline Clio........ & $1767 \mid$ pyramidata & L. & $\left.1767^{1}\right)$ \\
\hline Pall. & 1774 limacina ... & Phipes & $\left.1771^{1}\right)$ \\
\hline Trosch. & 1854 krolmii. & Trosch. & $\left.1854^{1}\right)$ \\
\hline RANG & 1828 acicula & Rang & $18281)$ \\
\hline Cymbulia .... VPÉr. \& Lst. & 1810 peronii & BLAINT. & $\left.1818^{1}\right)$ \\
\hline Desmopterus . C CHux & 1889 papilio & CHUx & $18891)$ \\
\hline La. & 1801 tridentata & ForsK. & 1775 \\
\hline (Cuv.) Las. & $\left.\begin{array}{l}(1817) \\
1819\end{array}\right)$ helicina & Phipps & $\left.1774^{1}\right)$ \\
\hline neumoderma . & 1804|peronii & Lis. & 1819i; \\
\hline
\end{tabular}

Lit.: 1) Tesch in: Tierreich, v. 361913.

Cephalopoda.

\begin{tabular}{|c|c|c|c|c|c|}
\hline Algonauta.... & L. & 1758 & argo & L. & 1758 \\
\hline Loligo.......... & Lir. & 1799 & vulgaris = lolige & Ext.. & 1799 \\
\hline Nautilus ....... & L. & 1758 & pompilius ..... & L. & 1758 \\
\hline Octopus ..... & Lar. & 1799 & rulgalis. & Lar. & 1799 \\
\hline Sepia ....... & L. & $175 \mathrm{~s}$ & officinalis. & I. & 1758 \\
\hline Spirula ....... & La. & 1799 & australis. . & LM. & 1816 \\
\hline
\end{tabular}

Lit.: P. Fischer, MIan. Conchyl., 1880-1887. 


\section{Prochorderta.}

Enteropneusta.

Balanoglossus . Ptychodera ...
Chlaje Eschz.
1829 clavigerus 1825 Hlava.
Chit.je 18291)

Eschz. $\left.\quad 1825^{1}\right)^{2}$ )

Spengel (Gießen).

\section{Pterolranchia.}

Cephalodiscus . M'INT. Althis.

W'INT.

Alilim. $\left.1882^{3}\right)$ $1869^{3}$ )

\section{Phoronidea.}

Phoronis...... Str. Wraght 1856|lippocrepia ... Str. Wright 18562) Lit.: 1) Spenged in: Zool. Jahrb. Syst., v. 151901.

2) Defage-Hérodard, T'raité Zool., v. 81898.

3) Harmer in: Siboga-Exp., par's $26^{2} 1905$.

\section{Tunicata.}

\section{Ascidicue.}

\begin{tabular}{|c|c|c|c|c|c|}
\hline Amaroucium ..' & II.-E. & $18+1$ & |proliferum .. & M.-E. & $\left.18+1^{1}\right)$ \\
\hline Aplidinm .... & $S_{\Lambda V}$ & 1816 & zostericola .... & GLARD & $\left.1872^{1}\right)$ \\
\hline Ascidia ....... & L. & 1767 & mentula ..... & MÜLL. & 1776 \\
\hline Ascidiella ..... & Rothe & 1883 & aspersa ...... & MÜLL. & $\left.1776^{1}\right)$ \\
\hline lia... & Siv. & 1816 & ovifera ....... & L. & 1767 \\
\hline Botryllus ... & C.F.GAERTA. & 1774 & schlosseri . . . & PALL. & $\left.66^{1}\right)$ \\
\hline & & & aurens .. & SARS & \\
\hline ison & FLED. & 1822 & intestinalis . . & $\begin{array}{l}\text { M.-L. } \\
\text { L. }\end{array}$ & $\left.1767^{1}\right)$ \\
\hline avenina... & SAV. & 1816 & lepadiformis ... & MULLL. & $\left.1776^{1}\right)$ \\
\hline orella.... & ALD. \& HANe. & 1870 & parallelo- & & \\
\hline$T_{x}$ & RASCHE & 1884 & $\begin{array}{r}\text { gramma } \\
\text { dellechiaiae ... }\end{array}$ & $\begin{array}{l}\text { MÜLL. } \\
\text { D. V VLLE }\end{array}$ & \\
\hline endrodoa & M'LEAX & 1825 & agoregata... . & RАтнкЕ & $\left.86^{1}\right)$ \\
\hline & & & grossularia... & Beneu. & $\left.6^{1}\right)$ \\
\hline$\ldots$ & SAT. & & viol & S.IV. & $61)$ \\
\hline ก11um . & $\mathrm{SAV}$ & & candidum ... & SAT. & $\left(6^{1}\right)$ \\
\hline ma . & MACDON. & 1859 & listerianum. . & MI.-E. & 41 \\
\hline Dis & D. VALLE & 1881 & magnilarva. . & D. TALLE & \\
\hline$S \ldots$ & C. F. Gaerta. & & variolo & C.F.GAERTN. & \\
\hline & ALD. \& HANC. & & arenosa. & ALD. \& HANC. & \\
\hline Halocynthia. . & TERRILI & 1879 & papillosa. . & Guxx. & 6.5 \\
\hline osmus & HELL. & 1877 & sulcatus .. & Coqueb. & 17971) \\
\hline Mc & Forb. & & oculata. & Fonв. & 48 \\
\hline ס1 .... & $\mathrm{II}^{\top} \mathrm{GM}$. & & listeri .... . & Forb. & \\
\hline Phallusia ..... & SAT. & & Imammillata. & Cut. & 15 \\
\hline
\end{tabular}




\begin{tabular}{|c|c|c|c|c|}
\hline Polycarpa. & Hell. & 1877 pomaria ... & $\mathrm{S}_{\mathrm{AV}}$. & 1816 \\
\hline Polycitor . & REN. & 1788 crystallinus & REN. & $\left.1788^{1}\right)$ \\
\hline Polyclinum. & $\mathrm{SAV}_{\mathrm{A}}$ & 1816 satunium. & SAY. & $\left.1816^{1}\right)$ \\
\hline Polyzoa ... & Less. & 1830 opuntia... & Less. & $\left.1830^{1}\right)$ \\
\hline Pyura. . & MoL. & 1782 chilensis & MOL. & $\left.1782^{1}\right)$ \\
\hline Sidnyum & SAY. & 1816 turbinatum. & SAY. & $\left.1816^{1}\right)$ \\
\hline & FLeMI. & 1822 canopus ... & SAY. & 1816 \\
\hline & LESS. & 1830 sigillinoides & LEss. & $\left.1830^{1}\right)$ \\
\hline & Phipps & $177+$ turgens.... & Рнipps & $\left.1774^{1}\right)$ \\
\hline Trididemnun & D. VALLEE & 1881 cereum . & GIARD & $\left.1872^{1}\right)$ \\
\hline
\end{tabular}

Lit.: Hartueyer in: SB. Ges. Freunde Berlin, 1915.

1) Hartieyer in: Brons's Kl. Ord., v. 3 suppl. p. 1280-1774 1909-11. Hartmeyer (Berlin), Michaflisen (Hamburg), Siluiter (Amsterdam).
Appendicularia
Cyclosalpa ....
Doliolum .....
Fritillaria.....
Pyrosoma.....
Salpa $\ldots . . .$.

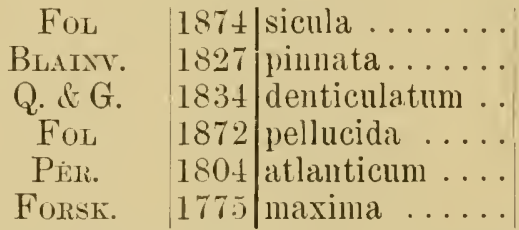
Lit.: 1) Lohmann in: Ergeb. Plankton Exp., v. $2 \mathrm{E}$ e 1896.
2) IHLe in: Tierreich. v. 321912.
3) G. Nedmann in: Tierreich, v. 401913.
A pstein (Berlin), Bongekt (Bonn), Farran (Dublin), Fowler (Aspley- Guise), Hartmeyer (Berlin), Mlerdan (Liverpool), Ihle (Utrecht), Lohmany (Hamburg), Mlichafisen (Hamburg), (t. Neumanx (Dresden), Ritter (La Jolla). Sugiter (Ansterdam). Todaro (Rom).

\begin{tabular}{c|c} 
Fol & $\left.187 t^{1}\right)$ \\
Forsk. & $\left.1775^{2}\right)$ \\
Q. \& G. & $\left.1834^{3}\right)$ \\
W. Busch & $\left.1851^{1}\right)$ \\
PER. & $\left.1804^{3}\right)$ \\
FURSK. & $\left.1775^{2}\right)$
\end{tabular}

T. pelagica*).

\title{
Cephalochordata.
}

\begin{abstract}
Amphioxus.... Yarr. 1836 planceolatus.... PaLL. 1774 Lit.: Delage-IIérouard, Traité Zool., v. 81898.
\end{abstract}

\section{Tertebrata.}

Pisces.

\begin{tabular}{|c|c|c|c|c|}
\hline Abramis . & Cots. & 1817|brama. & L. & 1758 \\
\hline Acanthias. & Risso & 1826 vulgaris & Risso & 1826 \\
\hline Acerina .. & Cer. & 1817 cermua. & L. & $175 \pi$ \\
\hline Acipenser & L. & 1758/sturio . & L. & 1758 \\
\hline Agomis. . & Bl. \& Scux. & 1801 cataphractus & L. & 1758 \\
\hline Alburnus & Heck. & 1840 lucidus . . . . & Heck. & 1858 \\
\hline Amia .... & L. & 1766 calva .... & L. & 1766 \\
\hline Ammodytes & & 17.8 tubianus. . & L. & 1758 \\
\hline Amplipirion & Bu. \& Scuis. & 1801 bifasciatus & Bl. & 1797 \\
\hline
\end{tabular}


Anabas . . . . . .

Annarlichas

Antemmarius .. . Comn. in LAc.

Argyropelecus .

Aspro .

Atherina

A ullastoma

Balistes

Barbus

Bdellostoma. .

Belone

Blennius

Brosmins

Calamoichthys .

Callionymus . . .

Callorhynchus

Canthar'us ....

Caranx .

Car*assius ....

Carcharias

Centriscus

Centroulutus ...

Cepola

Ceratodus

Cestracion

Chaetodon

Charax.

Chanliodus ....

Chiasmodus ...

Chimaera

Chondrostoma

Chrysophrys...

Clupea

Cobitis

Conger

Coregonts

Corvina

Coryphaena

Cottns. . .

Crenilabrus

Ctenolabrus

Cyclopterus

Cyprinodon

Cyprinus

Dactylopterus

Cuv.

Shaw

L.

Cocco

Cuv.

L.

Lac.

L.

Cuv.

J. MÜLL.

Cev.

L.

Cuv.

J. A. SiI.

L.

Cur.

Cur.

LAC.

Niliss.

Cut.

L.

BL. \& SchN.

L.

L. AG.

Cers.

L.

RIsso

Bl. \& SchN.

JoHxs.

L.

L. Ag.

Cuv.

L.

L.

Cuv.

Cuv.

Cuv.

L.

L.

Cuv.

Cuv. \& VaL.

L.

LAC.

L.

LAC. \begin{tabular}{l|l}
1817 & scandens \\
1804 & vulgaris
\end{tabular}

1758 lupus

1798

1829 hemigymnus . . .

1829 vulgaris

1758 hepsetus

1803 chinense .

1758 maculatus .

1817

1835

1817

17.58

1827

1865

1758

1817

1829

1802

1832

1817

1758

1801 gunellus

1758

1838

1817 plilippi

$1758 \mathrm{fas}$

1827

vulgaris

cirrhatum. .

acus

ocellaris

brosme.

yra

antarcticus.

lineatus

trachurus .

vulgaris

glaucus

scolopax

lubescens

1801

1863

17 -8

1758 monstrosa

1837

1829

1758 harengus

1758 fossilis

1817 vulgaris

1817 wartmanni

1829 nig'a

17581

1758

1817
1839 rupestris......

1758 lumpus ......

1803 calaritanus

1758 carpio

1802 volitans
DALD. 1797

Tere. 1807

L. $\quad 1758$

Cuv. \& VAL. 1837

Cocco 1829

Cur. 1829

L. $\quad 1758$

L. $\quad 175 \mathrm{~s}$

Gis. $\quad 1788$

Flent. 1828

BL. \& Schn. 1801

RIsso 1826

L. $\quad 1758$

MÜLL. $\quad 1776$

J. A. Sม. 1860

L. $\quad 1758$

LAC. 1798

Mont. 1815

L. $\quad 1758$

Nilss. 1832

L. $\quad 1758$

L. $\quad 1758$

L. $\quad 1758$

L. $\quad 1758$

KrefFt 1870

BL. \& Schn. 1801

Forse. 1775

GM.

1788

Bu. \& Schn. 1801

Johxs. 186.3

L. $\quad 1758$

L. $\quad 1758$

L. $\quad 1758$

L. $\quad 1758$

L. $\quad 1758$

RichaRds. 1844

Bu. $\quad 1784$

BL. $\quad 1797$

L. $\quad 1758$

L. $\quad 1758$

BRÜNN. 1768

L. $\quad 1758$

L. $\quad 1758$

Cur. \& VAL. 1846

L. 1758

GII. 


\begin{tabular}{|c|c|c|c|c|c|}
\hline Dentex. & Cur. & 1817 & |vulgaris . . . . . & Cur. \& VaL. & 1830 \\
\hline Diodon . . . . . . & L. & 1758 & hysstrix ...... & L. & 1758 \\
\hline Echeneis ..... & L. & 1758 & nancrates ..... & L. & 1758 \\
\hline Engraulis . . . . . & Cuv. & 1817 & encrassicholus . & L. & 1758 \\
\hline Esox . . . . . & L. & 1758 & lucius ........ & L. & 758 \\
\hline Exocoetus .... & L. & 1758 & evolans....... & L. & 1758 \\
\hline Fielasfer ..... & Cur. & 1817 & acus ........ & BRÜNN. & 1768 \\
\hline Fistularia..... & L. & 1758 & tabacaria ..... & L. & 8 \\
\hline Gadus........ & L. & 1758 & morrhua ...... & L. & \\
\hline & & & aeglefinus..... & L. & \\
\hline Galeus . . & Cor. & 1817 & canis........ & BOxAP. & \\
\hline Gasterosteus . & L. & 1758 & aculeatus ..... & L. & \\
\hline Gobio........ & Cut. & 1817 & fluviatilis ..... & FLEM. & \\
\hline Gobius ....... & L. & 1758 & niger ... . . . . & L. & \\
\hline Gymuotus.... & L. & 1758 & electricus .... & L. & \\
\hline Hippocampus . . & LFACH & 1814 & antiquorum ... & LEACH & \\
\hline Hippoglossus . . & Cor. & 1817 & rulgaris . . . . . & Flexi. & \\
\hline Histiopliorus . . & CUT. \& VAL. & 1831 & gladius . . . . . . & Brouss. & 6 \\
\hline Labrax ....... & Cur. & 1817 & lupus ......... & Cur. & \\
\hline Labrus . . . . . . & L. & 1758 & maculatus.... . & BL. & \\
\hline Lamna ....... & Cur. & 1817 & cornubica ..... & GM. & \\
\hline Lepidosiren ... & Fitz. & 1837 & paradoxa ..... & Fitz. & 337 \\
\hline Lepidosteus ... & LAC. & 1803 & platystomus ... & RAF. & \\
\hline Leuciscus . . . . . & Cur. & 17 & lutilus ....... & L. & \\
\hline Lophius ...... & L. & 1758 & piscatorius ... & $\mathrm{L}$. & 8 \\
\hline$\ldots \ldots \ldots$ & Cur. & 1829 & vulgaris . . . . . & JEN. & 835 \\
\hline Luciopelca. . . . & Cuv. & 829 & sandra ...... & Cuv. & \\
\hline Macropodus ... & $\mathrm{L}_{A C}$. & 1802 & viridi-auratus . & LAc. & \\
\hline Ма & BL. & 1787 & atlanticus.... & LowE & 39 \\
\hline pterurus. . & LAC. & 1803 & electricus .... & Gar. & 788 \\
\hline$\ldots \ldots$ & Ctr. & 17 & ertilio .... & L. & 758 \\
\hline Maurolicus .... & Cocco & 1838 & borealis ..... & Nitss. & \\
\hline Meluccius .... & RAF. & 1810 & vulgaris . . . . . & Fliem. & \\
\hline 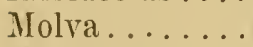 & LsR. & 1819 & rulgaris ..... & FLEM. & \\
\hline ylus .... & L. & 58 & lloides ... & L. & 758 \\
\hline Nugil . . . . . . . & L. & 58 & cephalus ...... & L. & \\
\hline Mullus ... . & L. & 1758 & barbatus..... & L. & \\
\hline$a \ldots$. & L. & 158 & lrelena....... & I. & 1758 \\
\hline istelus.... . & Cut. & 1817 & vulgaris ... . & J. MÜLL. \& & 1 \\
\hline Myliobatis & Dux. & 1817 & aqu & L. & 17 \\
\hline & L. & 1758 & glutinosa .. & L. & 175 \\
\hline tes.... & RAF. & 10 & ductor. . . . . . & L. & 175 \\
\hline optis..... & RAF. & 1810 & ophidion .... . & L. & 17 \\
\hline Notidanus.... & Cur. & 1817 & gliseus . . . . . . & Gu. & \\
\hline Ophidion ..... & I. & 1767 & barbatum ..... & L. & 1758 \\
\hline
\end{tabular}


Orthagoriscus Osmer'us .....

Ostracion

Pagellus ..... Cuv. \& VAL.

Pegasus

Pelamys

Perca

Periophthalmus

Petromyzon ...

Phoximus

Pleuronectus

Poecilia

Polyodon

Polypterus ....

Pomacentrus

Pristis

Protopterus

Raja

Regalecus.....

Rhinobatus.

Rhodeus

Rhombus

Saccobranchus

Saccopharynx

Salmo

Sargus

Scaphirhynchus

Scartus

Sciaena

Scomber

Scomberesox

Scopelus . .

Scorpaena

Scyllium . .

Sebastes

Selache.

Serranus

Silurus .

Siphonostoma. .

Solea

Squatina.

Synguathus ...

Tetrodon

Thymallus

Tinca Cuv.

L.

Bu. \& SchN.

L.

L. Ag.

L.

BL. \& SchN.

Ijac.

Geoffr.

LAC.

цатн.

Ow.

L.

NARDo

BL. \& SCHN.

L. Ag.

Lac.

TAL.

Мттен.

L.

Cuv.

Heck.

Forsk.

L.

L.

LAc.

Cuv.

L.

Cur.

Cuv.

Cur.

Cuv.

L.

RAF.

Cuv.

DUN.

L.

L.

Cuv.

Cuv.
Bu. \& Schn.

1817 eperlanus

1758 triqueter.

1830 ery thrinus

1758 volans.

Cuv. \& Vax.
1831 sarda

1758 fluviatilis

1801 koelreuteri

1758 fluviatilis

1837 laevis

1758 platessa

thesus

1801 vivipara

1798 folium .

1802 bichil

1802

1794

1837

1758

1827

1801

1836

1800

1840

1824

1758

1817

1835

1775

1758

17.58

1803

1817

1758

1829

1829

1817

1829

1758

1810

1817

1806 vulgaris

1758 acts

1766 lagocephalus

1829 vulgaris

1817/vulgaris
L.

L.

1758

L.

L.

L.

BL.

1758

1758

1758

L.

1758

Palis.

1797

L.

1758

L. Ag.

L.

L.

1770

1758

1837

1758

1758

BL. \& SchN. 1801

Lac.

1798

GeofFr.

1802

Cuv. \& Val. 1830

Latн.

1794

Ow.

1839

L.

1758

Cuv. \& Val. 1835

Cuv.

1829

BL.

1782

L.

1758

Cuv. \& VAL. 1840

Мттсн. 1824

L.

1758

Cuv. \& Vau. 1830

Grar

1834

L.

1758

Risso

1826

L.

1758

IVALB.

1792

RICH.

1844

L.

1758

L.

1758

MÜLL.

1779

Guns.

1765

L.

1758

L.

1758

L.

1758

Quensel

1806

RIsso

1810

I.

1758

L.

1758

Niuss.

1832

Cuv. 


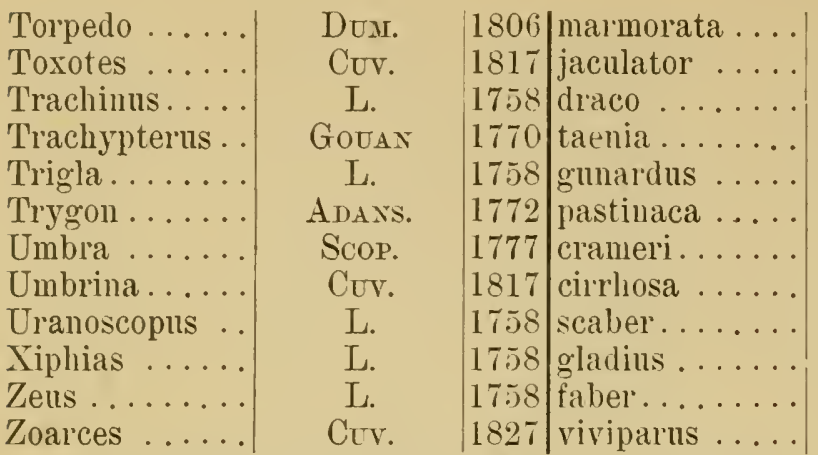

Lit.: Günther, Cat. Fish. Brit. Nus., v. $1-8$ 1859-70.
Risso 1810

PALL. 1770

L. $\quad 1758$

BL. \& S SнHк. 1801

I. $\quad 1758$

L. $\quad 1758$

J. MÜLL. 1842

L. $\quad 1758$

L. $\quad 1758$

L. 1758

L. 1758

L. $\quad 1758$

\section{Amphibia.}

Anura.

Alytes .......
Bombinator ...

Brericeps .....

Bufo .

Callula

Ceratophrys ...

Dendrobates ..

Discoglossus

Engystoma.

Hyla

Hylodes

Ixalus

Mantophryne . .

Megalixalus .

Megalophrys

Nototrema

Paludicola

Pelobates

Pelodytes

Phrynoba-

trachus.

Phyllomedusa

Pipa ..

Pseudis

Rana
NAGL.

Merr.

MERR.

LAUR.

GÜNTHER

(BOIE) WIED

IVAGL.

Оттн

Fitz.

LAUR.

Fitz.

Dux. \& Bibr.

BLGR.

GÜNTHER

KएHL

GÜxтHER

IVAGL.

IVAGL.

(FITZ.) BP.

GÜNTHER

W AGL.

LACR.

WAGL.

L. 1830|obstetricans ...

1820 ignens .

pachypus

1820 gibbosus .

1768 vulgaris

1864

1825

1830

1837

1826

1768

1826

pulchra.

cornuta

tinctorius

pictus

ovale

arborea.

martinicensis .

1841

1897

1868

1824

1858

1830

1830

1838

aurifasciatus .

lateralis ......

seychellensis

montana.....

marsupiatum .

albifrous.....

fuscus .......

punctatus

1862

1830

1768

1830

1758
LACR.

LACR.

(FItz.) BP.

$\mathrm{I}$.

LACR.

Griay

$\mathrm{L}$.

SCHNEID.

Оттн

Schineid.

$\mathrm{I}$.

(Tsch.) DणN. $\left.18+11^{2}\right)$

\& BIBR.

SCHL.

$\left.1839^{2}\right)$

BLAR. 18974)

Tsсн. 1838²)

КенL 182 $18 t^{2}$ )

Dux. \& Bibr. 18412)

SPIX

$\left.1824^{2}\right)$

LACR.

$\left.1768^{2}\right)$

DALD.

$1803^{2}$ )

A. SuI.

$\left.1859^{2}\right)$

BODD.

LAUR.

L.

L.

NIISS.

I.

A. Thomas $\left.1772^{2}\right)$

$1768^{2}$ )

$\left.1758^{2}\right)$

17581)

18+21)

$\left.1758^{1}\right)$

$\left.1855^{1}\right)$ 
Rappia ...... Günther |1864|marmorata... RAPP $_{\text {A }} 1842^{2}$ ) Rliacophorus .. (BoIE) Tsch. 1838 reinwardtii... . (BoIE) WAGL. 18302) Rhinoderma ... Dum.\&Bibr. 1841 dalwinii . . . . . Dum. \& Brbr. 18412) Xenopus..... Wagt. 1827/laevis....... Daud. 18032)

\section{Urodela.}

Amblystoma...(Tsch.) Cope 1838 |tigrinmm ...... Green 18253) Amphiuma .... Garden. 1821 means....... GARden. $1821^{3}$ ) Cryptobranchus F.S. Leuck. 1821 alleghaniensis . DAUD. 1803 ${ }^{3}$ ) Desmognathus .

BAIRD Megalobatrachus... Necturus Protens

Salamandra ...

Salamandrina. .

Siren .......

Spelerpes .....

Triton....... LAUR.

TSCH.
RAF.
LATR.
LATR.
Fitz.
L.
RAF.
LAUR.

1849 fuscus ....... RAF.

$1820^{3}$ )

ScHL. $1837^{3}$ )

RAF. $1819^{3}$ )

LAUR. $1768^{3}$ )

LAUR. $1768^{3}$ )

SAVI $1823^{3}$ )

L. $\quad 1767^{3}$ )

BР. $\quad 1837^{3}$ )

LAUR. $\quad 1768^{3}$ )

\section{Gymnophiona.}

Caecilia ........

Ichthyophis ...

Siphonops....

Typhlonectes . .

\begin{tabular}{|c|c|c|c|c|}
\hline L. & 1758 & |tentaculata.... & I. & $\left.1758^{5}\right)$ \\
\hline Fitz. & 1826 & glutinosus. . & I. & $\left.1758^{5}\right)$ \\
\hline :L. & 1828 & anmulatıs. & MIKAN & $\left.1820^{5}\right)$ \\
\hline Peters & 187 & compressicauda & Dũ. \& Bibr. & $\left..1841^{5}\right)$ \\
\hline
\end{tabular}

Lit.: 1) Boulenger, Taillous Batr. Eur., v. 21897.

2) Bodlenger, Cat. Batr. sal. Brit. II us, ed. 2 188\%.

3) Bodlenger, Cat. Batr. grad. Brit. Hus., ed. 21882.

4) Bodlenger in: Ann nat. Hist. ser. 6 v. 191897 .

5) Nieden in: Tierreich. v. 271913.

Nieden (Berlin), L. (t. Andersson (Stockholm), van Kampen (Amsterdam), E. Lönneerg (Stockholm), L. v. Méreley (Budapest), N. de Rooy (Amsterdam), J. Roux (Basel), R. Sternfeld (Frankfurt), Tornier (Berlin), Th. Vogt (Berlin), F. Werner (Wien).

\section{Reptitia.}

Ophidia (Lit. 4).

\begin{tabular}{|c|c|c|c|}
\hline Atractaspis & Lar. & 1849 |irregularis . & Reinh. \\
\hline Bitis ...... & GRAY & 1842 arietans ... & MERR. \\
\hline Boa.... & L. & 1766 constrictor. & L. \\
\hline Bungarus . . & DAUD. & 1803 candidus . . & L. \\
\hline Calamaria... & Bote & 1826 calamaria.... & L. \\
\hline Cerastes .... & WAGL. & 1830 cornutus ...... & L. \\
\hline Coluber. . . . & L. & 1766 longissimus ... & LAUR. \\
\hline Coronella ..... & LAUR. & 1768laustriaca .... & LAUR. \\
\hline
\end{tabular}


Crotalus ......

Dipsado-

morphus ....

Elaps .

Glauconia....

Hydrophis

Ilysia .......

Lachesis ......

Naia

Platurus

Psammophis..

Python .......

Tropidonotus .

Typhlops .....

Uropeltis

Vipera

Xenopeltis

Zamesis

\begin{tabular}{|c|c|c|}
\hline L. & 1766 & horridus . \\
\hline$W_{A G L}$. & 1830 & scabra ...... \\
\hline FITZ. & 1826 & irregularis \\
\hline YHNEID. & 1801 & fulvius . . . \\
\hline GRAY & 1845 & albitrons... \\
\hline $\mathrm{D}_{\mathrm{AUD}}$ & 1803 & fasciatus.... \\
\hline HEMPR. & 1820 & scytale .... \\
\hline DAUD. & 1803 & mutus ... \\
\hline LAUR. & 1768 & haje .... \\
\hline LATR. & 1802 & laticaudatus. . \\
\hline BoIE & 1827 & sibilans.... \\
\hline DAUD. & 1803 & molurus . . \\
\hline Кчнц & 1824 & natrix ...... \\
\hline CHNEID. & 1801 & lumbricalis . . \\
\hline & & grandis. . \\
\hline LAUR. & 1768 & berus ...... \\
\hline REINW. & 1827 & unicolor $\because .$. \\
\hline WAGL. & 1830 & gemonensis . . . \\
\hline
\end{tabular}

\begin{tabular}{c|c} 
L. & 1766 \\
L. & 1766 \\
MEIR. & 1802 \\
L. & 1766 \\
WAGL. & 1824 \\
SCHNED. & 1799 \\
L. & 1766 \\
L. & 1766 \\
L. & 1762 \\
L. & 1766 \\
L. & 1766 \\
L. & 1766 \\
L. & 1766 \\
L. & 1766 \\
KELAART & 1853 \\
L. & 1761 \\
REINW. & 1827 \\
LAUR. & 1768
\end{tabular}

\section{Hydrosauria et Chelonia (Lit. 5).}

Alligator .....

Caiman

Chelone

Chelydra

Chelys

Cinixys . ......

Clemmys .....

Crocodilus ....

Dermochelys . .

Emys .......

Gavialis

Hatteria

Pelomedusa

Sternothaerus

Testudo

Thalassochelys

Trionsx ......

\section{Cuv.}

Sptx

BROGN.

SCHWEIGG.

Dun.

BELL

IVAGL.

LAUR.

Blainv.

E. DUM.

Cuv.

GraY

WAGL.

BELL

L.

FIтz.

Geofrr.
1807 /mississipiensis .

1825

180.5

1814

1806

1827

1830

1768

1816

1806

1807

gangeticus ....

1842 puuctata......

1830 galeata . . . . . . . .

1825 nigricans .....

1766 giraeca

1835) caretta

1809 ferox.
DAUD. 1802

SchNED. 1801

L. $\quad 1768$

L. $\quad 1766$

SCHNEID. 1783

BeLL 1827

Gมr. 1774

LAUR. 1768

\begin{tabular}{c|c} 
L. & 1766 \\
L. & 1766 \\
GM. & 1789 \\
GRAI & 1842 \\
SCHOEPFF & 1792 \\
BELL & 1825 \\
L. & 1766 \\
L. & 1768 \\
SCHNEUD. & 1783
\end{tabular}

Sauria.

Agama .......

Algiroides .... Amblyrhynchus Amplisbaena..

\begin{tabular}{c|r|l|} 
Daud. & 1802 & stellio . . . . . . \\
Bibr. & 1833 & moreoticus . . . \\
Bert & 1825 & clistatus . . . . \\
L. & 1766 & alba . . . . . .
\end{tabular}

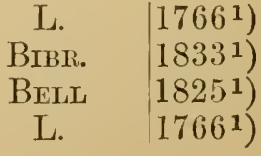


Anguis Anolis.

Basiliscus .... .

Calotes

Chalcides .....

Clıamaeleon ...

Chlamydosaurus

Draco .

Egernia

Eremias

Eublepharis

Gecko

Gerrlıosaurus .

Gymmodactylus

Heloderma ... .

Hemidactylus

Iguana

Lacerta

Lygosoma ....

Mabuya .....

Metopoceros...

Moloch .

Plielsuma

Phrynosoma...

Physignatis...

Pygopus......

Scincus

Tarentola.....

Teius

Tiliqua

Tupinambis

Uromastix

Uroplatus

Varanus

Zonnrus
L.

DAUD.

LAUR.

Cuv.

LAUR.

LAUR.

GRAY

L.

Gray

TVGI.

Gray

GraY

IVGI.

Spix

IVGM.

Gray

LAUR.

L.

GRAY

Fitz.

II ${ }_{\text {AGL. }}$

GRAY

Gray

IVGM.

Cur.

MerR.

LATR.

Gray

Merr.

Gray

DAUD.

MerR.

Dus.

MerR.

MERR.
1766 fragilis .

$1803 \mathrm{p}$

1768 a

1829 versicolor . . . . .

1768 tiic

1768

1827 ,

1766

18.38

1834 velox

1827 hardwickii

1825 verticillatus ...

1828 Havigularis

1825 geckoides .

1829 horridum

1825 turcicus

1768 tuber culata

1766 agilis

1828 chalcides

1826 sloanii.

1830 cornutus

1841 horridus

1825

1828

1829 cochinchinensis

1820 lepidopodus ...

1768

1825 man itanica ...

1820 teyou

1825 gigas.

1803 tegiuxin

1820 spinipes

1806 fimbriatus

1820 niloticus

1820 cordylus
L.

$\left.1766^{1}\right)$

DAUD.

LAUR.

DAUD.

LAUR.

L.

Griar

L.

LAC.

Pall.

Gray

LAUR.

ITGM.

SPIX

WG Gr.

L.

LATR.

I.

$I_{1}$.

DAUD.

DAUD.

Grar

Gray

L.

Cur.

LaC.

L.

L.

DAUD.

Schneid.

L.

DAUD.

SCHNEID.

L.

L. $\left.1803^{1}\right)$

$\left.1768^{1}\right)$

$\left.1802^{1}\right)$

$1768^{1}$ )

$1758^{3}$ )

$\left.1845^{1}\right)$

$1766^{1}$ )

$1804^{1}$ )

$\left.1771^{1}\right)$

18271)

17681)

18281)

$1825^{1}$ )

$1829^{1)}$

$\left.1766^{1}\right)$

$\left.1768^{1}\right)$

$\left.1766^{1}\right)$

$\left.1766^{1}\right)$

1803 1 )

18031)

18411)

$1825^{1}$ )

$1766^{1}$ )

$\left.1829^{1}\right)$

$1804^{2}$ )

$\left.1766^{1}\right)$

$\left.1766^{1}\right)$

18031)

1801 1 )

$\left.1766^{1}\right)$

18031)

$1797^{2}$ )

$1766^{1}$ )

$1766^{1}$ )

Lit.: 1) Boulenger, Cat. Lizards Brit. Hus., ed. 2 v. $1-3$ 1885-87.

2) Whrner in: Tierreich, v. 331912.

3) Werner in: Tierreich, v. 271911.

4) Boulenger, Cat. Snakes Brit. Mus, v. 1-3 1893-96.

5) Boulenger, Cat. Chelon. Brit. Mus., ed. 21889.

S'Ternfeld (Frankfurt) /Ophidia, Sauria?, Nieden, Tornier, Th. Vogt (Berlin).

Aves.

Accentor

Bechst.

1802 Briss.

Accipiter

NAUM.

1760 nisus .

Gir.

I.

L. 
Aepyornis .... Alauda ....... Alca.

Alcedo

Amazona

Anas.

Anastomus ....

Anser .......

Anthus

Aptenodytes...

Apteryx .

Aquila

Ardea

Arenalia

Asio

Astur

Athene

Attagis .

Balaeniceps ...

Bubo.

Bucco

Bucelos

Buteo

Caccabis

Cancroma .

Capito

Caprimulgus...

Cariama

Casuarius

Centropus

Certliia.

Charadrius

Chamma . .

Chionis

Ciconia

Cinclus

Circus

Coccothraustes.

Colins

Collocalia .....

Columba .

Colymbus

Conurus

Coracias

Corvus
J. Geofrr. '1851 |maximus .

\begin{tabular}{ll|l} 
L. & 1758 & alvensis \\
L. & 1758 & torda . \\
L. & 1758 & ispida .
\end{tabular}

Less.

L.

Boxwat.

Briss.

Bechst.

Forst.

SHAW

Briss.

I.

Briss.

Briss.

LAC.

BoIf

Less. \&

J. GEOFFr.

J. GD.

DŨ.

Briss.

L.

LAC.

KAUP

L.

VIEILL.

L.

Briss.

BrIss.

ILL.

I.

L.

ILL.

Forst.

1831 farinosa

1758 boschas.

1790 oscitans

1760 anser

1807 trivialis

1781 patachonica ...

1813

1760 chrysaetos

1758 cinerea

1760 int

1760 otus

1801

1822 noctua

1830 gayi

1851 lex

1806 bubo

1760 collaris

1758

1799 buteo

1829 lufa

1766 cochlearia

1816

1758 ellopaeus.....

1760 cristata.

1760 casualius

1811 senegalensis .

1758 familiaris

1758 pluvialis

1811 cliavaria

Briss.

1788 alba

1760 ciconia

Borkhaus.

Lac.

Briss.

Briss.

G. R. GRAY

L.

L.

KัH

I.

I.
1797 cinclus

1799 a eruginosus

1760 coccothraustes

1760 colius

1840 esculenta

1758 oenas

1758 cristatus

1820 leucophthalmus

1758 garrulus

1758 colax
J. GEOFFR.

L.

L.

L.

BoIn.

L.

BODI.

I.

L.

Forst.

SHAW

L.

L.

L.

L.

L.

Scop.

Less.

J. GD.

L.

LATH.

L.

L.

L.

L.

ST. MÜLL.

L.

I.

L.

L.

L.

I.

I.

Gir.

L.

I.

I.

L.

I.

I.

I.

L.

ST. MÜLL.

I.

L.
1851 3 )

$1758^{2}$ )

17581)

$\left.1758^{2}\right)$

178:31)

$17581)$

178. $3^{1}$ )

$\left.1758^{1}\right)$

$\left.1758^{2}\right)$

$\left.1781^{1}\right)$

181:31)

$\left.17.58^{2}\right)$

17.581 )

$\left.1758^{1}\right)$

$\left.1758^{1}\right)$

17581)

$\left.1769^{2}\right)$

$\left.1830^{1}\right)$

18.511)

$\left.1758^{2}\right)$

$\left.1790^{1}\right)$

$\left.1758^{1}\right)$

$\left.1758^{2}\right)$

$17581)$

$\left.1766^{1}\right)$

$\left.1776^{1}\right)$

$\left.1758^{2}\right)$

$\left.1766^{1}\right)$

$\left.1758^{1}\right)$

$176(; 1)$

$\left.1758^{2}\right)$

$\left.1758^{1}\right)$

$17\left(66^{1}\right)$

$\left.1788^{1}\right)$

$\left.1758^{2}\right)$

$\left.17.58^{2}\right)$

$\left.17.58^{2}\right)$

$\left.1758^{1}\right)$

$\left.1766^{1}\right)$

$17.981)$

17581)

175810)

$\left.1776^{1}\right)$

$\left.1758^{2}\right)$

$\left.1758^{2}\right)$ 
Cotinga .....

Coturnix ....

Crax ........

Grex ........

Crotophaga ...

Crepturus.....

Cuculus

Cursorius .....

Cyginus .

Cypselus......

Dendrocopos . .

Dicrurus .

Didunculus . . . .

Didus

Dinornis .....

Diomedea .....

Dromains .

Dromas .

Eclectus ......

Ectopiste

Emberiza

Erithacus

Eurypyga

Falco

Francolinus ...

Fregata ......

Firingilla

Fulica

Furnarius

Galbula

Gallinago

Gallinula

Gallus.. .

Garrulus

Glareola.

Grus

Gypes

Gypaetıs

Haematopus . .

Halcyon

Haliaetus

Himantopus ...

Hirundo

Hippolais

Ibis . .
Briss.

BonNat.

I.

BеснST.

L.

ILL.

L.

LАтн.

Bеснsт.

ILL.

K. L. Косн

VIEILL.

Peale

I.

Ow.

L.

VImLLL.

PAYK.

WAGL.

Sw.

L.

Cer.

ILL.

I.

STEPH.

BRISS.

L.

L.

VIEILL.

Briss.

LFaCH

Briss.

Briss.

Briss.

Briss.

PALL.

Sit.

STORR

L.

Sw.

S.tY.

Briss.

L.
1760 cotinga .

1791 coturnix

1758 rubra

1802

1758 an

1811 cinereus

1758 canor'ts.

1790 gallicus .

1803

1811

1816 major .

1817 balicassius

1814 strigirostris

1766 ineptus

1e 43 novae-zealandiae

1758 exulans.

1816 novaehollandiae

1805 ardeola

1832 pectoralis

1827 migratorius

175 citıinella

1800 rubecula

1811 helias

1758 subbuteo .

1819 francolimus

1760 aquila

1758 coelebs

1758 atra

1816 rufus.

1760

1816

1760

1760

1760

1760

1766

1809

1784

1758 ostralegus

1820 senegalensis

1809 albicilla

1760 himantopus

1758 rustica

Chr.L.Brehi 1828 icterina

Crr.
L.

L.

L.

L.

L.

Gir.

L.

GiI.

Gis.

L.

L.

L.

JARD.

L.

OW.

L.

цатн.

PAYK.

ST. MÜLL.

L.

L.

L.

Pall.

L.

L.

L.

L.

L.

Gir.

L.

Frisch

L.

L.

L.

I.

L.

HABL.

L.

L.

Less.

L.

I.

L.

Tieill.

LATH. $\left.1766^{1}\right)$

17581)

17581)

$1758^{1}$ )

17581)

17881)

$1758^{2}$ )

$\left.1788^{1}\right)$

17881)

17581)

17584)

$1766^{1}$ )

$\left.1845^{1}\right)$

$\left.1766^{1}\right)$

$\left.1843^{5}\right)$

$\left.1758^{1}\right)$

17901)

$\left.1805^{1}\right)$

$\left.1776^{1}\right)$

17661)

$\left.1758^{2}\right)$

$1758^{6}$ )

17811)

$1758^{2}$ )

$\left.1766^{1}\right)$

17581)

$1758^{2}$ )

17581)

17881)

$\left.1766^{1}\right)$

$1763^{1}$ )

$17581)$

17581)

17581)

$\left.1766^{1}\right)$

17581)

17831)

$\left.1758^{2}\right)$

$\left.1758^{1}\right)$

$1831^{2}$ )

$17.582)$

$17581)$

17587)

$\left.1817^{2}\right)$

$1790^{1}$ ) 
Icterus .

Indicator

Jynx . .

Lagopiss

Lamprocolius .

Lanius

Larus .

Leptoptilos

Limosa

Lorius

Loxia

Luscinia .

Meleagris . .

Nemua.

Mer'gus

Merops

Milvus

Mimus

Monticola

Motacilla

Muscicapa

Nectarinia

Neophron

Nestor

Notornis .

Numenius ....

Numida

Nycticorax....

Oedicnemus ...

Opistlocomus .

Oriolus .......

Otis

Palaeornis

Palamedea

Paladisea....

Parus

Pandion

Passer

Pelecanus

Perdix

Permis.

Phaethon

Phalacrocorax .

Phalaropus....

Phasianus.....

Phoenicopterus

BRISS.

VIEILL.

L.

Briss.

Stad.

L.

L.

Less.

Briss.

VIG.

L.

Fonst.

I.

Davies

L.

L.

LAC.

BoIE

Bure

L.

Briss.

ILL.

SAv.

Less.

Ow.

Briss.

I.

Raf.

T'емx.

ILL.

L.

L.

VTT.

L.

L.

I.

Siv.

Briss.

Briss.

BrIsS.

Cer.

L.

BrIsS.

BuIs:.

L.

l.
1760 icter'us

1816

1758 torquilla .

1760

1835 phoenicopterus.

1758 excubitor

1758 cantus

1831 javanicus

1760 limosa. . .

1825 domicella

1758 curvirostra....

1817 Inscinia .

1758 gallopavo

1800

1758 merganser

1758 apiaster

1799 milvus .

1826 polyglottos ....

1822 saxatilis

1758 alba

1760 grisola

1811 famosa

1809 percuopteris . .

1831 meridionalis

$18 \pm 8$ mantelli ...

1760 arquatus.

1760 meleagris

1815

1815 oedicnemus

1811 hoazin.

1766 oriolus

1758 tarda

1828 empatria.

1766 cornuta.

1758 apoda

1758 major

1809 haliatus ....

1760 domesticus ....

1760 onocrotalus ...

1760 perdix .

1817 apivortus.

1758 aethereus

1760 carbo . .

1760 lobatus

1758 colchicus

1758 iruber
L.

$1766^{1}$ )

Gr. 17881)

L.

$\left.1758^{2}\right)$

L. $\left.1758^{1}\right)$

Sw. 18371)

L. $\quad 1758^{2}$ )

L. $\quad 1758^{1}$ )

HoRsf. 18211)

L. $1758^{1}$ )

L. 17581)

L. $17.58^{2}$

L. $17.58^{12}$ )

L. 17581)

DAVIES $1800^{1}$ )

L. $\quad 1758^{1}$ )

L. $\quad 1758^{2}$ )

L. $\quad 1758^{2}$ )

L. $1758^{1}$ )

L. $\left.\quad 1766^{2}\right)$

L. $1758^{2}$ )

L. $\left.\quad 1766^{8}\right)$

L. $\left.\quad 1766^{1}\right)$

L. $\quad 1758^{2}$ )

Gr. 17881)

(W. $1848^{1}$ )

L. $\quad 1758^{1}$ )

L. $\quad 17581$ )

L. 17581 )

L. 17581)

ST. MÜLL. $\left.1776^{1}\right)$

L. $\quad 1758^{2}$ )

L. 17581 ,

L. $\quad 1766^{1}$ )

L. $\left.\quad 1766^{1}\right)$

L. $\left.\quad 1758^{1}\right)$

L. $\quad 1758^{2}$ )

L. $\left.17.58^{2}\right)$

L. 17581)

L. 17.881)

L. 17581)

L. $\quad 1758^{2}$ )

L. 17.581)

L. 17581)

L. 17581)

L. $17.58^{1}$ )

L. 17581) 
Phylloscopus ...

Phytotoma ....

Pica

Picus

Pitta

Platalea

Plocens

Porphyrio...

Pratincola

Procellaria ....

Psittacus

Psophia

Puffinus

Pyrrhula......

Rallus

Ramphastos...

Recurvirostua

Regulus. .

Rhea

Sarcorhamphus

Saxicola ......

Scolopax

Scopus.

Serpentarius

Sitta

Somateria .

Spheniscus

Steatornis.

Stercorarius...

Sterna ........

Strigops ......

Strix

Struthio

Sturnus

Sula

Sylvia

Syrnimm

Syrutiaptes

Syrthaptes ....

Tanag'a

Tantalus .

Tetrao.

Thinocorus ....

Tichodroma

Tinamus

Totanus. .

Trichoglossus
Bore

MOL.

Briss.

L.

VIEILL.

L.

Cuv.

Briss.

K. L. КосH

L.

L.

I.

Briss.

Briss.

I.

L.

L.

VIEILL.

Briss.

Dus.

Bechst.

L.

Gir.

Cur.

L.

LEICH

Briss.

Humboldt

Briss.

L.

G. R. GRAY

L.

L.

L.

BRISS.

Scop.

SAY.

ILL.

L.

I.

I.

Eschz.

ILL.

J. HeRM.

BECHST. 1826|trochilus ......

1782 lara .

1760 pica

1758 viridis

1816 brachyura.....

1758 lencorodia.

1817 baya

1760 porphyrio .

1816 rubetra

1758 aequinoctialis

1758 erithacus.

1758 crepitans

1760 putfinus.

1760

1758 aquaticus

1758

1758

1807

1760

1806

1802

1758 rusticola.

1788 umbret ta .

1798

1758

1819

1760

1810

1760

$1758 \mathrm{l}$

184.5

1758

1758

1758

1760

1769

1809 aluco

1811

1766 episcopus

1758 loculator

1758 urogallus

1829 rumicivorus

181

1783

muraria......

1803 totanns

1803 to
L.

MoL.

L.

I.

L.

L.

BLyth

I.

L.

I.

L.

L.

BRÜNN.

L.

L.

L.

L.

L.

I.

L.

L.

L.

Giv.

$\left.1758^{2}\right)$

$1782^{1}$ )

$1758^{1}$ )

175811)

$\left.1766^{1}\right)$

$\left.1758^{1}\right)$

$\left.1844^{1}\right)$

17581)

$1758^{2}$ )

$\left.1758^{1}\right)$

17581)

$1758^{1)}$

176t')

$\left.1758^{1}\right)$

17581)

$1758^{1)}$

$\left.1758^{1}\right)$

$1758^{2}$ )

$\left.1758^{8}\right)$

17581)

$\left.1758^{2}\right)$

$\left.1758^{1}\right)$

$\left.1788^{1}\right)$

G. S. MILL.

L.

I.

L.

$\left.178.5^{1}\right)$

$\left.1758^{2}\right)$

17581)

17581)

Humbotat 1810 1 )

'T'ena.

L.

$\left.1815^{1}\right)$

17.581)

G. R. GRAY 1845')

L.

L.

$\left.1766^{7}\right)$

I.

17581)

L.

$17582)$

$176(51)$

LATH.

L.

Patl.

L.

L.

L.

Eschz.

L.

Gir.

$\left.1787^{2}\right)$

17581)

17731)

17661)

17581)

17581)

$\left.1829^{1}\right)$

$\left.1766^{2}\right)$

I.

$\left.1788^{5}\right)$

I.

17581)

17711) 
Triinga .........

Troglodytes ...

Trogon

Thracus

T'urdus

Turtur.

Tyramus ......

Upupa $\ldots . . . . .$.

Uria $\ldots . . . . . . .$.

Vanellus

Vidua

viltur.

\begin{tabular}{|c|c|c|}
\hline L. & 1758 & alpina \\
\hline VIEILL. & 1807 & troglodytes.... \\
\hline L. & 1766 & viridis . . . . . . . \\
\hline Cur. & 1800 & macrorhynchus \\
\hline L. & 1758 & viscivorus .... \\
\hline SeLby & 1835 & turtur ... \\
\hline Cor. & 1800 & tyrannus. \\
\hline L. & 1758 & epops..... \\
\hline Briss. & 1760 & troille ... \\
\hline BRIss. & 1760 & vanellus .... \\
\hline Cuv. & 1800 & serena ..... \\
\hline BRISS. & 176 & monaclius ... \\
\hline
\end{tabular}

\begin{tabular}{c|l} 
L. & $\left(1758^{1}\right)$ \\
L. & $\left.\left.1758^{6}\right)^{2}\right)$ \\
L. & $\left(1766^{1}\right)$ \\
FrAS. & $\left.1839^{1}\right)$ \\
L. & $\left.1758^{2}\right)$ \\
L. & $\left.1758^{1}\right)$ \\
L. & $\left(1758^{1}\right)$ \\
L. & $\left.1758^{2}\right)$ \\
L. & $\left.\left(161^{1}\right)^{9}\right)$ \\
L. & $\left(158^{1}\right)$ \\
L. & $\left(1766^{1}\right)$ \\
L. & $\left.1766^{1}\right)$
\end{tabular}

Lit.: $\left.{ }^{1}\right)$ Cat. Birds Brit. Mus, v. 1-27 1874-1895.

2) Hartert, Vögel pal. Eauna., 1903- . . .

3) Lydekker, Cat. foss. Birds Brit. Mlus, 1891.

4) Hesse in: Urn. Monber., 1912 p. 160-162.

5) Sharpe, Hand-L. Birds, v. 1 -5) $1899-1909$.

6) W aterhouse, Index (ien. Avium. 1889.

7) Reichenow in: Orn. Mouber., 1913 p. 173, 174.

8) Reichenow, Handb. syst. Orn., v. 1, 2 1913, 1914.

9) Reichenow. Kemnz. Vögel Deutschl., 1902

10) Reichenow in: J. Oru., 1889 p. 188.

11) Cabanis \& Heine, Mus. Hein., v. 41863.

12) Oberholser in: The Auk, 1906 p. 228.

Reichenow (Berlin), Hesse (Berlin).

\section{Mammalia.}

Ailurus

Alces

Alloa...

Anthropopithe-

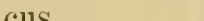

Antilope .......

Arctictis

Aretomys

Arvicola

Ateles.

Auchenia

Babirissa......

Balaena

Balaenoptera ..

Bathyergus

Bisoli

Bos

Bradypus

Bubalis. . .

Camelus

Canis
F. Cur.

GruY

H. SM.

Buant.

P.LLL.

Temas.

Schier.

Lac.

GEOFFr.

ILL.

RAF.

L.

Lic.

ILL.

I1. Sir.

I.

I.

L' 'HT.

I.

L.
1825. fulgens . ... 1821 |1827 depressicornis

1838

1766 cervicapra ....

$182+$

1780

1801

1806

1811 glama .........

1815)

175.

17.58 mysticetus ...

1804

1811 maritimus......

1827

1758

1758 trids .........

181. tridactylus....

1814 bubalis

1758 dromedarius....

1758 familiaris
F. Cov.

L.

H. SII.

Geoffr. 1812 L.

1758

RAFHL.

Schreb.

1822

I.

1780

I.

17.58

L.

1758

I.

L.

1758

MÜLI.

Giv.

L.

I.

L.

1758

1758

PALI.

I.

L.
1776

1788

1758

1758

1758

1767

1758

1758 
Capra

Capromys .....

Cariacus

Castor .

Catodon.

Cavia

Cebus

Centetes.

Cercolabes

Cercoleptes.

Cercopithecus

Cerrits

Chiromys

Choloepus .

Chrysochloris.

Coelogenys ....

Colobus .

Condylura.....

Commochaetes.

Cricetus ......

Crocidura.....

Crossopus*) ...

Cystophora....

Dasyprocta

Dasypus ......

Dasyurus

Delphinus .

Dicotyles

Didelphis

Dipus...

Echidna

Elephas

Enhydris.

Equus

Erethizon

Erinaceus

Eriomys

Felis

Galago

Galeopithecus

Gazella

Globicephalus

L.
Desil.
Less.
L.
L.
PALI.
ERIL.
ILL.

Brandt

ILL.

Errit.

L.

Cuv.

ILL.

LAC.

ILL.

ILL.

IILL.

Lснт.

LESKE

IVAGL.

IVAGL.

Nillis.

ILL.

L.

E. Geoffr.

L.

Cuv.

L.

Ziмm.

Cuv.

L.

J. B. Fisch.

F. Cuv.

Lснт.

L.

GeofFr.

PALL.

Lснт.

LESS.
1758 hircus

1822 fommieri

1842 virginianus . . . .

1758 fiber

1761 macrocephalus.

1766 cobaya ......

1777 capucinus

1811 ecaudatus .....

1811 ecaudatus .....

1835 prehensilis ....

1811 caudivolvulus.. PALt.-

1778 nictitans

1758 elaplıus

1800 madagasca-

liensis . . .

1811 didactylus .....

1799 auratus

1811 paca. .

1811 polycomus.....

1811

1812

1770

1832 leucodon ......

1832 fodiens.......

1820 cristata .......

1811 aguti .

1758 sexciuctus.....

1796 viverrinus

1758 delphis

1817 tajaçu

1758 marsupialis

1780 sagitta .

1798 aculeata

17.58 maximus

1829 lntris .

1758 caballus

1822 dorsatus

1758 europaeus .

1829 lanigera

1758 catus .

1796 galago

1780 volans

1812 doreas

1842|melas
Schreis.

L.

1758

Desit.

1822

BODD.

1785

I.

L.

1758

PALL.

1761

L.

G.I.

L.

1766

1758

1788

1766

1777

L.

L.

1758

1758

Gir.

1788

L.

1766

Cuv.

1798

L.

1766

Schreb.

1775

L.

1758

Zinน.

L.

HERM.

Schreb.

Erxu.

L.

L.

Shaw

L.

L.

L.

PALL.

Shaw

L.

L.

L.

L.

L.

MoL.

L.

Schreb.

L.

L.

TraILI 1809

*) Gegen: Neomys Kaup 1829. 
Gorilla........ Gulo .

Halichoerus ...

Hapale

Herpestes .... .

Hippopotamus .

Hippotragus. .

Hyaena ......

Hydrochoerus

Hydromys.....

Hylobates.....

Hyperoodoll ...

Hystrix .......

Lagidium

Lagostomus ...

Lemur........

Lepus

Lutra.........

Lynx

Macacus ......

Macropus .....

Manatus

Manis

Meles

Mephitis ......

Midas.

Monodon ......

Moschus .....

Mus

Mustela

Mycetes

Myodes

Myopotamus .

Myotis .......

Myoxus

Myrmecobius .

Myrmecophaga

Nasua .......

Nycteris .....

Nycticebus*)..

Nyctipithecus

Ochotona

Ornitho-

rhynchus ...

Is. GEOFFr. $1852 \mid$ gorilla .......

PaLL.

NULLS.

ILL.

ILL.

L.

SUND.

BODD.

Enxu.

GEOFFR.

ILL.

Lac.

L.

MEXEN

Ввоок.

L.

L.

ERXL.

KERR

DESAI.

Sнаш

ScOP.

L.

STORR

Cuv.

Geofrr.

L.

L.

L.

L.

ILL.

PALIs.

Geoffr.

KaUP

Zами.

WATERH.

L.

STORR

Geofrr.

GeOFFr.

SPIX

LINK

Blunib.
1780 gulo .

1820 grypus

1811 jacchus

1811 ichneumon

1758 amphibins

1846 lencophaeus

1785 hyaena

1777 liydrochoerus..

1805 chrysogaster ..

1811

1804

1758 cristata......

1833 peruanum .....

1828 trichodactylus .

1758 catta

1758 timidus

1777 lutra .

$1792 \operatorname{lynx}$

1820 rhesus

1790 giganteus

1777 manatus

1758 pentadactyla ..

1780 meles ........

1800 mephitis .....

1812 rosalia ...

1758 monocertus ....

1758 moschiferus ...

1758 musculus ......

1758 martes.

1811 seniculus ......

1811 lemmus .......

1805 coypus . .

1829 myotis .

1780 glis

1836 fasciatus .......

1758 tridactyla .....

1780 nasua.........

1814 hispidus

1812 coucang.

1823 trivirgatus ....

1795 pusillus .

1800|paradoxus .
WGMr.

$18 \pm 7$

L. $\quad 1758$

F.

1791

I.

1758

L. $\quad 1758$

L. $\quad 1758$

PALL. $\quad 1766$

L. $\quad 1758$

L. $\quad 1766$

GEOFFr. 1805

Gy. $\quad 1788$

Bonnat. 1789

L. $\quad 1758$

Neyen 1833

Brook. 1828

L. $\quad 1758$

L. $\quad 1758$

L. $\quad 1758$

L. 1758

L. $\quad 1758$

Shaw $\quad 1790$

L. $\quad 1758$

L. $\quad 1758$

L. $\quad 1758$

Schreb. 1776

L. $\quad 1767$

L. $\quad 1758$

L. $\quad 1758$

L. $\quad 1758$

L. $\quad 1758$

L. 1758

L. $\quad 1758$

Geofrr. 1805

BeCHST. 1801

L. $\quad 1758$

W ATERH. 1846

L. $\quad 1758$

$\begin{array}{ll}\text { L. } & 1758 \\ \text { S } & 1790\end{array}$

BODD. 1785

HUNB. 1811

GALL 1778

BLUNB. 1800

*) Gegen: Bradicebus Cuv., Georrr., 1795. 
Orycteropus ...

Otaria

Ovibos

Ovis .

Papio.........

Pedetes.

Perameles

Petaurus .

Phacochoerus

Phascolarctos

Phascolomys .

Phoca ........

Phocaena

Phyllostomus . .

Pithecia

Plecotus

Potamochoerus

Potorous*) ....

Procavia......

Procyon

Pteromys .....

Pteropus

Putorius

Rangifer ......

Rhinoceros....

Rhinolophus...

Rupicapra ....

Rytina.......

Sarcophilus ...

Sciurus .......

Semnopithecus

Simia ........ .

Sorex.........

Spalax

Spermophilus**)

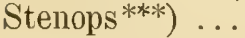

Strepsiceros ...

Sus .

Synotus $†) . . .$.

Talpa.........

Tapirus . .

Tardigradus...
GEOFFR.

Pér.

Blainv.

L.

Enxu.

ILL.

GEOFFr.

Shaw

F. Cuv.

Blainv.

GEofrR.

L.

Cuv.

Lac.

DESM.

GEOFFr.

GRAY

DESM.

STORR

STORR

Cuv.

Enxน.

Cuv.

H. Sur.

L.

Lad.

BLAINV.

ILL.

F. Cuv.

L.

Cuv.

L.

L.

GÜLD.

Cuv.

ILL.

RAF.

L.

KEys.-BL.

L.

Scop.

BODD.
1803 capensis .....

1816 ju

1816 moschatus....

1758 aries

1777 papio

1811 cafer

1804 nasuta

1791 australis

1817 aethiopicus ....

1816 cinereus ......

1803 ursinus

1758 vitulina ......

1817 phocaena

1799 hastatus

1804 pithecia

1813 auritus

1854

1804 tridactylus

1780 capensis

1780

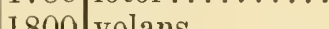

1800 volans

1777 vamipylus .....

1817 putorius

1827 tarandus

1758 unicornis .

1799 ferrum equinum

1816 rupicapra .....

1811 gigas

1837 ursinus

1758 vulgaris

1825 entellus

1758 satyrus

1758 araneus

1770 typhlus

1825 citellus

1811 gracilis

1815 strepsiceros ...

1758 scropha .

1839 barbastellus

1758 europaea.....

1777 terrestris

1784 loris
GEOFFR. 1803

Forst. 1775

Zам. 1780

L. $\quad 1758$

Desm. $\quad 1820$

Pall.

GEOFFR.

1778

SHaw

L.

GoLDF.

Shaw

L.

L.

PALL.

L.

L.

L.

KERR

Pall.

L.

L.

L.

L.

L.

L.

Schreb.

L.

Zimar.

STORR

L.

DUFR.

L. (part)

L.

PALL.

L.

GEOFFR.

Pall.

L.

Schred.

L.

L.

1804

1791

1768

1819

1791

1758

1758

1767

1758

1758

1758

1792

1766

1758

1758

1758

1758

1758

1758

1774

1758

1780

1780

1758

1797

1758

1758

1779

1766

1796

1766

1758

1774

1758

1758

BODD.

*) Gegen: Hypsiprymnus ILL. 1811.

**) Gegen: Citellus OKen 1816.

***) Gegen: Loris Geoffr. 1796.

†) Gegen: Barbastellus Gray 1821. 
Tarsius ....... STORR

Thylacinus ....

Tragulus ......

Trichechus ....

Tupaja ........

Ursus

Vespertilio ....

Viverra.......

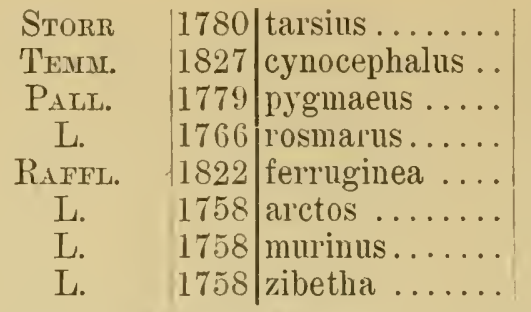

ERxL. HARr. 1808 BODD. $\quad 1785$ L. $\quad 1766$ RAFFL. $\quad 1822$ L. $\quad 1758$ L. $\quad 1758$ L. $\quad 1758$

Lit: Palmer, Index Gen. Mam., 1904.

Bem.: Die Korrekturen für die auswärtigen Herrn Mitarbeiter hat der Herausgeber besorgt.

\section{Zweite wissenschaftliche Sitzung am 18. Iai 1915.}

A. Hase-Jena: Zur Biologie der Kleiderläuse, nach Beobachtungen im Quarantänelager Hammerstein. 



\section{Auszug aus den Gesetzen}

der

\section{Gesellschaft Naturforschender-Freunde}

\section{zul Berlin.}

Die im Jahre 1773 gestiftete Gesellschaft Naturforschender Freunde in Berlin ist eine freundschaftliche Privatverbindung zur Beförderung der: Naturwissenschaft, insbesondere der Biontologie.

Die Gesellschaft besteht aus ordentlichen, auferordentlichen und Ehremmitgliedern.

Die ordentlichen Mitglieder, deren Zahı höchstens 20 betragen darf, ergänzen sich durch einstimmige Wahl nach den dmeh königliche Bestätigung vom 17. September 1789 und 7. Februar 1907 festgestellten Gesetzen. Sie verwalten das Vermögen der Gesellschaft und wählen aus ihrem Kreise die Vorsitzenden und Schatzmeister.

Die anßerordentlichen Mitglieder, deren Zahl unbeschränkt ist, werden von den ordentlichen Mitgliedern, auf Vorschlag eines ordentlichen Mitgliedes unter eingehender Begründıng, gewählt. Für freie Zustellung der Sitzungsberichte mad Einladungen zu den Sitzungen zahien die außerordentlichen Mitglieder einen Jahresbeitrag von 5 Mark. Sie kömen das "Archiv für Biontologie" und alle von der Gesellschaft unterstiitzten Veröffentlichungen zum ermäßigten Preise bezielıen.

Die wissenschaftlichen Sitzungen finden mit Ansuahme der Monate August und September an 2. und 3. Dienstage jedes Monats bis anf weiteres im Hörsaale VI, bzw. im Konferenzzimmer der Kgl. Landwirtschaftlichen Hochschule Invalidenstr: 42, abends 7 Uln, statt.

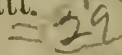

Alle für die Gesellschaft bestimmten Sendungen sum an den Sekretiar, Herrn Dr. K. Griuberg, Berlin N 4. Invalidenstr. 43, zu richten. 
$x \quad \frac{2}{2}$

WaSH

$\Sigma$

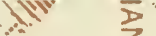

$\sum$

Noshas
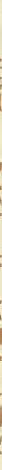

NOILOLILSNI NHINOSHLIWS

LIBRARIES SMITHSONIAN

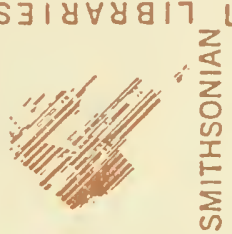
IBRARIES

SMITHSONIAN
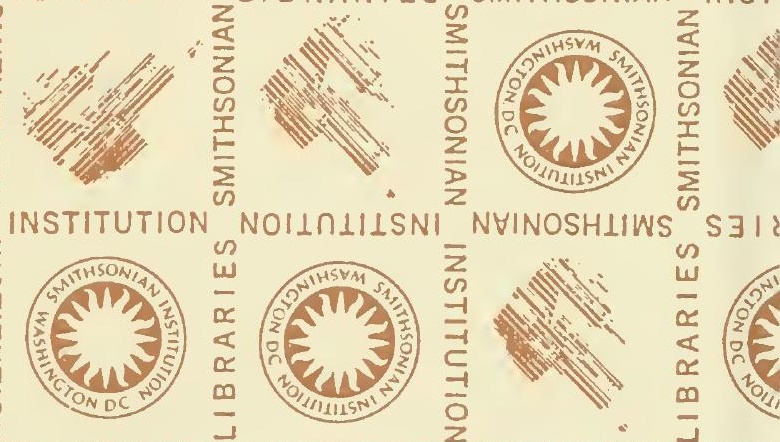

NHINOSHLIWS
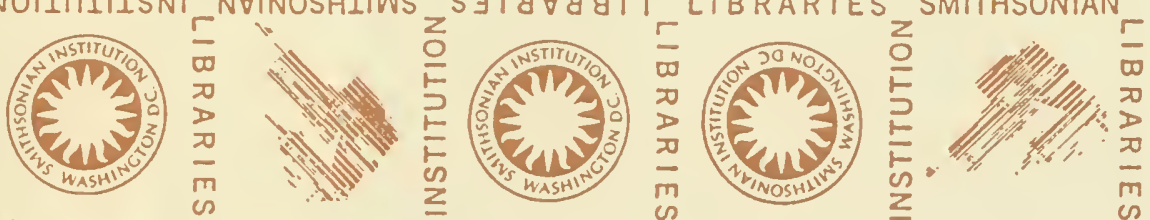

INST
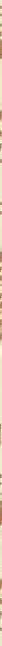

NVINOSHIIWS

IBRARIES SMITHSONIAN INSTITUTION

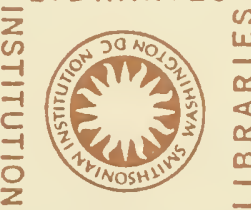

SMITHSONIAN

INSTI
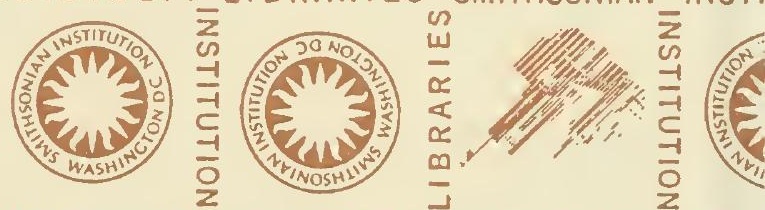

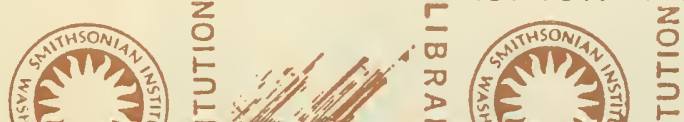
NOIINLIISNI NVINOSHLIWS $5 \exists 18$ 
SMITHSONIAN INSTITUTION LIBRARIES 